\title{
FUNCTOR OF POINTS AND HEIGHT FUNCTIONS FOR NONCOMMUTATIVE ARAKELOV GEOMETRY
}

\author{
ALICIA LIMA \& MATILDE MARCOLLI
}

\begin{abstract}
We propose a notion of functor of points for noncommutative spaces, valued in categories of bimodules, and endowed with an action functional determined by a notion of hermitian structures and height functions, modeled on an interpretation of the classical functor of points as a physical sigma model. We discuss different choices of such height functions, based on different notions of volumes and traces, including one based on the Hattori-Stallings rank. We show that the height function determines a dynamical time evolution on an algebra of observables associated to our functor of points. We focus in particular the case of noncommutative arithmetic curves, where the relevant algebras are sums of matrix algebras over division algebras over number fields, and we discuss a more general notion of noncommutative arithmetic spaces in higher dimensions, where our approach suggests an interpretation of the Jones index as a height function.
\end{abstract}

\section{INTRODUCTION}

The use of noncommutative geometry methods in Arakelov geometry was originally introduced in [12], [13, with a description of the special fiber at infinity of arithmetic surfaces in terms of a noncommutative space related to the geometry of Schottky uniformization of Riemann surfaces. A formalism for Arakelov geometry for noncommutative arithmetic curves and surfaces, based on arithmetic vector bundles and height functions, was later developed in [6], [7]. This second approach is based on noncommutative projective algebraic geometry in the sense of Artin-Zhang [3], while the results of [12, 13] used noncommutative differential geometry in the sense of Connes. In particular, the use of [3] limits the approach of [6], 77 to the cases of arithmetic curves and surfaces. The question of whether there is a good way to connect these two approaches and include higher dimensional arithmetic noncommutative spaces is mentioned in [7]. One of the goals of the present paper is to establish a formalism that adapts the viewpoint of [6] and [7] to work in a setting compatible with noncommutative geometry in the sense of Connes and covering arithmetic noncommutative spaces of arbitrary dimension.

Our formalism to describe arithmetic noncommutative spaces is based on the following main ideas:

- It is possible to have a good notion of functor of points for noncommutative spaces, provided the usual set-valued notion of $S$-points of a scheme $X=$ $\operatorname{Spec}(R)$ given by $X(S)=\operatorname{Hom}_{\mathrm{Alg}}(R, S)$ is replaced by a functor with values 
in categories, that replaces the set $\operatorname{Hom}_{\mathrm{Alg}}(R, S)$ with the category of $R-S$ bimodules.

- The dual notion of functor of points as $\pi_{X}: \operatorname{Sch}^{o p} \rightarrow$ Sets with $\pi_{X}(Y)=$ $\operatorname{Hom}_{\text {Sch }}(Y, X)$ can be envisioned as a $\sigma$-model with target $X$, where the points, given by maps $\phi \in \operatorname{Hom}_{\mathrm{Sch}}(Y, X)$, are weighted by an action functional measuring their energy, in the form of a height function.

- Suitable height functions can be defined for bimodules, and they give rise to a dynamical system on a convolution algebra of bimodules with the tensor product operation, with a partition function that corresponds to a height zeta function.

- The Jones index of Hilbert $C^{*}$-bimodules of finite type can be seen as a possible height function for noncommutative spaces.

In recent years extensions of $\sigma$-models to noncommutative spaces have been studied in various contexts, sometime motivated by string theory (see for instance [26], [44]), sometimes by extending results on the geometry of solitons to the noncommutative framework (see for instance [16]). As shown in [14], [15], several interesting new phenomena occur in the noncommutative setting and even very simple cases, such as the example of a target space consisting of a two-point space can have highly nontrivial solutions.

Here we take a different viewpoint. We broadly regard $\sigma$-models as describing dynamics on a space of maps between two assigned geometries. Instead of the usual Riemannian viewpoint, however, we start from a different commutative setting, consisting of the functor of points of algebraic geometry.

In order to make the functor of points "dynamical", we need to assign a suitable "action functional" to points of an affine scheme. We argue that a good measure of the "energy" of points (seen as maps between affine schemes) is provided by the height function. The idea that height functions should be regarded as a physical action functional, with the height zeta function playing the role of the partition function of the system, was already suggested by Manin in [38].

We adapt this viewpoint to the noncommutative setting. It is well known that an analogous notion of functor of points for noncommutative rings is problematic, due to the scarcity of two-sided ideals, hence of non-trivial ring homomorphisms (points in the classical sense). It is also well known, however, that in noncommutative geometry morphisms of algebras are not the most natural choice of morphisms of noncommutative spaces, and bimodules are a much more natural choice, in view of phenomena such as Morita equivalence, which provides the appropriate notion of isomorphism for noncommutative spaces. Thus, we propose replacing the functor of points of affine schemes with a functor from the category of (noncommutative) rings with morphisms given by bimodules, with values not in the category of sets, but in the 2-category of small categories. Under this noncommutative "functor of points" the "S-points" of a ring $R$ form the category of $R-S$ bimodules. 
We then seek an appropriate replacement for the height function that makes this notion of points dynamical. We present two different proposals for a height function on bimodules. The first is based on extending to our setting a notion of height developed for noncommutative arithmetic curves in [6] and based on volumes associated to hermitian forms on bimodules. We show that this notion of height carries over to our setting but it has the drawback that it is not always compatible with the composition operation given by the tensor product of bimodules. The compatibility can be restored by restricting to a suitable subcategory of bimodules. The second form of height function that we consider is naturally compatible with tensor products of bimodules and is based on the Hattori-Stallings rank. We show that, when generalized from noncommutative arithmetic curves to higher dimensional noncommutative arithmetic spaces, this second notion gives rise to an interpretation of the Jones index as a height function.

The paper is organized in the following way. In \$2 we review some known facts about height functions that serve as background motivation for what follows and justify our thinking of the height as an action functional. In $\$ 3$ we introduce our proposal for a functor of points in noncommutative geometry that uses categories of bimodules instead of sets of ring homomorphisms. In we focus on the case of finite-dimensional semisimple algebras, and correspondingly of noncommutative arithmetic curves. We show that the height function used in [6] can be generalized to our setting, but is only compatible with tensor product of bimodules if we restrict it to a specific subcategory. We propose an alternative notion of height, based on the Hattori-Stallings rank, which is better behaved under tensor product of bimodules and we discuss the time evolutions generated by these height functions seen as energy functionals. In $\$ 5$ we introduce a setting describing arithmetic noncommutative spaces in higher dimension and their functor of points, using hermitian bimodules of finite type. We consider as an example, arithmetic structures on noncommutative tori. We show that the two notions of height discussed in the case of finite dimensional algebras extend to this setting. The natural generalization of the height based on the Hattori-Stallings rank uses the Jones index of Hilbert $C^{*}$-bimodules.

\section{Functor of Points and Height Dynamics}

Let $X$ be an affine scheme over a field $\mathbb{K}$. We will assume that $\mathbb{K}$ is number field. In fact, for our purposes we can just take $\mathbb{K}=\mathbb{Q}$. Points of the scheme $X$ are defined through the functor of points, from the opposite category of schemes to sets

$$
\pi_{X}: \operatorname{Sch}^{o p} \rightarrow \text { Sets, } \quad \pi_{X}(Y)=\operatorname{Hom}_{\mathrm{Sch}}(Y, X) .
$$

The set $\pi_{X}(Y)$ describes the $Y$-points of $X$. An equivalent way of formulating the functor of points is dually in terms of the corresponding algebras. Let $R$ be a commutative $\mathbb{K}$-algebra with $X=\operatorname{Spec}(R)$. We then consider

$$
\pi_{X}^{\vee}: \operatorname{Alg}_{\mathbb{K}} \rightarrow \text { Sets, } \quad \pi_{X}^{\vee}(S)=\operatorname{Hom}_{\mathrm{Alg}_{\mathbb{K}}}(R, S),
$$


where $\operatorname{Alg}_{\mathbb{K}}$ is the category of commutative algebras over $\mathbb{K}$. The set $\pi_{X}^{\vee}(S)$ is the set of $S$-points of $X$. This includes for example, the case of algebraic points of $X$, that is $X(\overline{\mathbb{Q}}):=\pi_{X}^{\vee}(\overline{\mathbb{Q}})$. It is customary to simply use the notation $X(S)$ for the set $\pi_{X}^{\vee}(S)$ of $S$-points of the scheme $X$, for a $\mathbb{K}$-algebra $S$ and we will adopt this notation too.

More concretely, one can think of an affine scheme $X$ over the field $\mathbb{K}$ as solutions to a set of polynomial equations $f_{1}\left(x_{1}, \ldots, x_{n}\right)=0, \ldots, f_{N}\left(x_{1}, \ldots, x_{n}\right)=0$ for a given set of polynomials in $\mathbb{K}\left[x_{1}, \ldots, x_{n}\right]$. If we identify the scheme with the system of equations, then the set $X(S)$ of $S$-points of $X$ for a $\mathbb{K}$-algebra $S$ represents the set of solutions to these polynomial equations with $x_{i} \in S$. (The set of $n$-tuples of elements of the algebra $S$ that satisfy the system of polynomial equations.) This description of the scheme $X$ is clearly equivalent to describing it through the $\operatorname{ring} R=\mathbb{K}\left[x_{1}, \ldots, x_{n}\right] / \mathcal{I}_{X}$ with the ideal $\mathcal{I}_{X}=\left(f_{1}, \ldots, f_{N}\right)$. Any solution with $x_{i} \in S$ defines a morphism $R \rightarrow S$, hence we recover the previous description of the functor of points. Thinking in terms of systems of equations, we then describe morphisms of affine schemes as polynomial transformations between the sets of solutions of two different systems of equations. In other words, if $Y$ is determined by equations $g_{1}\left(y_{1}, \ldots, y_{r}\right)=0, \ldots, g_{L}\left(y_{1}, \ldots, y_{r}\right)=0$ and $X$ by equations $f_{1}\left(x_{1}, \ldots, x_{n}\right)=0, \ldots, f_{N}\left(x_{1}, \ldots, x_{n}\right)=0$, a morphism $\sigma: Y \rightarrow X$ can be described as a set of polynomials $\ell_{1}\left(y_{1}, \ldots, y_{r}\right), \ldots, \ell_{n}\left(y_{1}, \ldots, y_{r}\right)$ such that, if $\left(s_{1}, \ldots, s_{r}\right)$ with $s_{i} \in S$ is a solution to the first system, a point in $Y(S)$, then $\ell_{1}\left(s_{1}, \ldots, s_{r}\right), \ldots, \ell_{n}\left(s_{1}, \ldots, s_{r}\right) \in S$ give a solution to the second system, a point in $X(S)$. Equivalently the substitution of variables $\left(x_{1}=\ell_{1}\left(y_{1}, \ldots, y_{r}\right), \ldots, x_{n}=\right.$ $\left.\ell_{n}\left(y_{1}, \ldots, y_{r}\right)\right)$ defines a morphism $\mathbb{K}\left[x_{1}, \ldots, x_{n}\right] \rightarrow \mathbb{K}\left[y_{1}, \ldots, y_{r}\right]$ that descends to the quotients $R_{X}=\mathbb{K}\left[x_{1}, \ldots, x_{n}\right] / \mathcal{I}_{X} \rightarrow R_{Y}=\mathbb{K}\left[y_{1}, \ldots, y_{r}\right] / \mathcal{I}_{Y}$ with $\mathcal{I}_{X}=\left(f_{1}, \ldots, f_{N}\right)$ and $\mathcal{I}_{Y}=\left(g_{1}, \ldots, g_{L}\right)$. We spelled out morphisms explicitly in this way, since this will be useful in the next subsection.

A scheme is determined up to isomorphism by its functor of points. For a general introduction to the geometry of schemes we refer the reader to [37.

2.1. Height as an Action Functional. We consider here the functor of points as providing the kinematics, namely the space of maps to the target space given by the scheme $X$ from a (variable) source space given by the scheme $Y$. In order to make this model dynamical, we need to specify an action functional with respect to which the maps $\sigma \in \operatorname{Hom}_{\mathrm{Sch}}(Y, X)$ are weighted.

We recall a few facts about the height function. We refer the reader to [5] for a detailed introduction. As above let $\mathbb{K}$ be a number field with $d=[\mathbb{K}: \mathbb{Q}]$. Let $\mathcal{P}_{\mathbb{K}}$ be the set of places of $\mathbb{K}$, with $\mathcal{P}_{\mathbb{K}}=\mathcal{P}_{\mathbb{K}}^{a r} \cup \mathcal{P}_{\mathbb{K}}^{\text {nar }}$ where $\mathcal{P}_{\mathbb{K}}^{a r}$ is the set of archimedean places, consisting of $r$ real embeddings $\mathbb{K} \hookrightarrow \mathbb{R}$ and $k$ conjugate pairs of complex embeddings $\mathbb{K} \hookrightarrow \mathbb{C}$ (not contained in $\mathbb{R}$ ) with $d=r+2 k$, and $\mathcal{P}_{\mathbb{K}}^{\text {nar }}$ is the set of non-archimedean places. For $\nu \in \mathcal{P}_{\mathbb{K}}$ we write $\mathbb{K}_{\nu}$ for the corresponding local field (an extension of a $p$-adic field $\mathbb{Q}_{\nu}$ at the non-archimedean places and a copy of $\mathbb{R}$ or $\mathbb{C}$ at the archimedean ones). Let $d_{\nu}=\left[\mathbb{K}_{\nu}: \mathbb{Q}_{\nu}\right]$ be the degree of the extension at the non-archimedean places, and equal to either 1 or 2 at the archimedean cases that 
are either real or complex. We also write $|x|_{\nu}$ for the corresponding absolute values normalized so as to satisfy the product formula $\prod_{\nu \in \mathcal{P}_{\mathbb{K}}}|x|_{\nu}^{d_{\nu}}=1$.

The height of an algebraic number $\alpha \in \overline{\mathbb{Q}}$ is defined as

$$
H(\alpha)=\prod_{\nu \in \mathcal{P}_{\mathbb{K}}} \max \left\{1,|\alpha|_{\nu}\right\}^{d_{\nu} / d},
$$

and the logarithmic height as

$$
h(\alpha)=\log H(\alpha)=\sum_{\nu \in \mathcal{P}_{\mathbb{K}}} \frac{d_{\nu}}{d} \log ^{+}|\alpha|_{\nu}
$$

with $\log ^{+} t=\log \max \{1, t\}$. Here $d$ and $d_{\nu}$ and the set of places $\mathcal{P}_{\mathbb{K}}$ are taken with respect to a choice of a finite extension $\mathbb{K}$ of $\mathbb{Q}$ that contains $\alpha$, though the resulting value is independent of this choice. The height of an algebraic number satisfies $h(\alpha)=0$ iff $\alpha$ is a root of unity.

In a similar way, one can define the height of a polynomial. One defines for

$$
\begin{gathered}
f\left(x_{1}, \ldots, x_{n}\right)=\sum_{k_{1}, \ldots k_{n}} \alpha_{k_{1}, \ldots, k_{n}} x_{1}^{k_{1}} \cdots x_{n}^{k_{n}} \in \mathbb{K}\left[x_{1}, \ldots, x_{n}\right] \\
h(f)=\sum_{\nu \in \mathcal{P}_{\mathbb{K}}} \frac{d_{\nu}}{d} \log |f|_{\nu}, \quad \text { where } \quad|f|_{\nu}=\max _{k_{1}, \ldots, k_{n}}\left|\alpha_{k_{1}, \ldots, k_{n}}\right|_{\nu} .
\end{gathered}
$$

In fact, it is customary to replace in the archimedean contribution to the height $h(f)$ in the above formula the term $|f|_{\nu}$ with the better behaved Mahler measure

$$
M(f)=\exp \left(\frac{1}{(2 \pi)^{n}} \int_{\mathbb{T}^{n}} \log \left|f\left(e^{i \theta_{1}}, \ldots, e^{i \theta_{n}}\right)\right| d \theta_{1} \cdots d \theta_{n}\right) .
$$

Indeed, while at the non-archimedean places one has $|f g|_{\nu}=|f|_{\nu} \cdot|g|_{\nu}$ this multiplicativity does not hold at the archimedean places, while the Mahler measure satisfies $M(f g)=M(f) M(g)$.

Given two affine schemes $X$ and $Y$ and a morphism $\sigma \in \operatorname{Hom}_{\mathrm{Sch}}(Y, X)$, we can then define a height of $\sigma$, by identifying as in the previous section the morphism $\sigma$ with a collection of polynomials $\ell_{1}\left(y_{1}, \ldots, y_{r}\right), \ldots, \ell_{n}\left(y_{1}, \ldots, y_{r}\right)$ that transform by change of variables $S$-solutions of the polynomial equations defining $Y$ to $S$-solutions of the polynomial equations defining $X$. We then define, for the vector $\ell=\left(\ell_{i}\right)_{i=1}^{n}$ in $\mathbb{K}\left[y_{1}, \ldots, y_{r}\right]$ the quantity $\|\ell\|_{\nu}=\max _{i=1, \ldots, n}\left|\ell_{i}\right|_{\nu}$ and we compute the height $h(\sigma)$ as in (2.5), (2.6) using this quantity (with $M\left(\ell_{i}\right)$ instead of $\left|\ell_{i}\right|_{\nu}$ at the archimedean places).

The definition above provides us with a real valued action functional

$$
h: \operatorname{Hom}_{\mathrm{Sch}}(Y, X) \rightarrow \mathbb{R},
$$

with $h(\sigma)$ given by the height of the morphism $\sigma$. This means that we are thinking of the height as measuring the "energy" of the points in $X(S)$ for $Y=\operatorname{Spec}(S)$. 
It is reasonable to think of the height as an energy functional. For example, the Mahler measure determines the free energy and the growth rate of BPS states in toric quiver gauge theories, [59]. Mahler measure of certain bivariate polynomials arises as the free energy in the planar dimer model, [28], for Mahler measures and dimer models see also [54]. The Mahler measure is also related to instanton expansion, [55]. The height function can be viewed as a measure of arithmetic complexity, hence minimizing an action functional based on height means selecting points of minimal arithmetic complexity.

2.2. The minimization problem. With our choice of energy functional (2.7) then looking for energy minimizers means minimizing the height function over systems of polynomials $\ell=\left(\ell_{i}\right)_{i=1}^{n}: \mathbb{A}^{r} \rightarrow \mathbb{A}^{n}$, subject to the constraints imposed by the source and target schemes $Y$ and $X$, namely that the polynomial map $\ell$ descends to a map of the quotients $\ell: \mathbb{K}\left[x_{1}, \ldots, x_{n}\right] / \mathcal{I}_{X} \rightarrow \mathbb{K}\left[y_{1}, \ldots, y_{r}\right] / \mathcal{I}_{Y}$.

However, it is clear by looking at the archimedean contribution to the action functional given by the Mahler measure that if we want to obtain a non-trivial and interesting minimization problem for this energy functional, we need to be more restrictive in the choice of our class of maps. Indeed, it is better to work under the assumption that our schemes are defined over $\mathbb{Z}$ (or the ring of integers of a number fields). Let us assume for this discussion that they are defined over $\mathbb{Z}$. This means that we only need to consider polynomials with $\mathbb{Z}$ coefficients, both in the defining equations of our schemes and in the morphisms between them,

$$
\ell: \mathbb{Z}\left[x_{1}, \ldots, x_{n}\right] / \mathcal{I}_{X} \rightarrow \mathbb{Z}\left[y_{1}, \ldots, y_{r}\right] / \mathcal{I}_{Y}
$$

We are then seeking to minimize the height over such polynomials. That this is now an interesting minimization problem can be seen already in the case where both $X=Y=\mathbb{A}^{1}$ and a single polynomial map $\ell: \mathbb{A}^{1} \rightarrow \mathbb{A}^{1}$. In this case the Mahler measure $M(\ell) \geq 1$ and it is known that equality holds whenever $\ell(x)$ is a product of cyclotomic polynomials and the monomial $x$. So these realize the minima of the Mahler measure. More interesting is the question of what are the polynomials with the smallest value of the Mahler measure that is $M(\ell)>1$. This is known as the Lehmer problem, see [20, [18, [46]. Lehmer noted that the polynomial $\ell(x)=$ $x^{10}+x^{9}-x^{7}-x^{6}-x^{5}-x^{4}-x^{3}+x+1$ has $M(\ell)=1.1762808 \ldots$ and the question of whether this is an optimal lower bound is an open question. This shows that indeed there are interesting minimization questions associated to the action functional we are considering.

It is also worth pointing out the following aspect of this problem. Consider a scheme $X$ and its set of rational points $X(\mathbb{K})$. It is known that the distribution of rational points of bounded height is very not uniform: these points accumulate on subsets that are the range of certain morphisms. In this case then the question becomes the search for algebraic points of minimal height (for the appropriate version of the height function). 
2.3. The case of correspondences. This commutative case is discussed here only as motivation, hence we do not discuss in further detail this minimization problem. We do, however, discuss a variant of it, where morphisms $\sigma: Y \rightarrow X$ are replaced by more general correspondences, given by subschemes $\Gamma \subset Y \times X$. The case of a morphism $\sigma: Y \rightarrow X$ is recovered as the particular case where $\Gamma_{\sigma} \subset Y \times X$ is the graph of the morphism $\Gamma_{\sigma}=\{(y, \sigma(y)) \in Y \times X \mid y \in Y\}$. Passing from morphisms given by maps of algebraic varieties or schemes to morphisms given by correspondences is a very natural step in algebraic geometry and it is crucial in the development of the theory of motives. For us, the reason why it is useful to extend the setting discussed above from maps to correspondences lies in the fact that morphisms given by correspondences are the commutative analog of morphisms given by bimodules in noncommutative geometry (see a discussion of this in Chapter 4 of [10]). Passing from morphisms of varieties to correspondences is very delicate, since one usually needs to consider correspondences up to some notion of equivalence relation. This is related to the fact that the composition of correspondences is given geometrically by an intersection product: for $\Gamma_{1} \subset Z \times Y$ and $\Gamma_{2} \subset Y \times X$ the composite $\Gamma_{2} \circ \Gamma_{1} \subset Z \times X$ is obtained by pulling back both correspondences to the triple product $Z \times Y \times X$ via the respective projections, intersecting them, and pushing the result forward to $Z \times X$,

$$
\Gamma_{2} \circ \Gamma_{1}=\pi_{Z \times X *}\left(\pi_{Z \times Y}^{*}\left(\Gamma_{1}\right) \bullet \pi_{Y \times X}^{*}\left(\Gamma_{2}\right)\right) .
$$

We do not discuss this in detail here, but some of the subtleties involve having to work with smooth projective varieties rather than affine schemes, having the freedom to move representative algebraic cycles describing the correspondences within an equivalence class that preserves intersection numbers, etc. All these technical aspects are worked out in the construction of categories of (pure) motives, where morphisms are described in this way. We will not deal directly with motives here, and we simply refer the reader to [2] for a detailed introduction.

For our purposes, it suffices to point out that the notion of height of a morphism $\sigma: Y \rightarrow X$ that we discussed above, based on measuring the height of a polynomial, can be generalized to a notion of height of a variety, which can then be applied to a correspondence $\Gamma$, seen as a subvariety of $Y \times X$. We are making here a simplifying assumption and considering varieties instead of more general schemes. Notions of height function for motives are discussed in [27].

We will not be dealing directly with correspondences in the commutative case, but we mention this setting as a comparison, because our approach to the noncommutative case, which we will be discussing shortly, is based on the analog of correspondences for noncommutative spaces, which is given by bimodules.

\section{Bimodules and a Noncommutative Functor of Points}

When we move from the commutative to the noncommutative world, the notion of functor of points as usually constructed in algebraic geometry is clearly inadequate. If 
$R$ and $S$ are noncommutative $\mathbb{K}$-algebras, there are in general very few algebra homomorphisms $\phi: R \rightarrow S$, hence defining a functor of points using $\operatorname{Hom}_{\mathrm{Alg}_{\mathbb{K}}}(R, S)$ gives very little information. There are various ways in which noncommutative geometry has considered the problem of a good definition of points. A widely used approach consists of replacing the notion of points as algebra homomorphisms with the notion of points as extremal measures (delta measures supported on points in the classical commutative case). In noncommutative geometry, this involves the notion of states on the algebra of functions. In the case of a complex involutive unital algebra $R_{\mathbb{C}}$, a state is a linear functional $\varphi: R_{\mathbb{C}} \rightarrow \mathbb{C}$ that is normalized $\varphi(1)=1$ and satisfies a positivity condition $\varphi\left(a^{*} a\right) \geq 0$ for all $a \in R_{\mathbb{C}}$. These two properties generalize to the noncommutative setting the notion of a measure. In contrast to the set of algebra homomorphisms, which tends to be too small in the noncommutative case, the set of extremal points of the convex set of states tends to be too large, but this problem is usually cured by introducing additional requirement, for example only considering special states that are equilibrium (KMS) states for a dynamical evolution of the noncommutative algebra. This approach was successfully used in applications of noncommutative geometry to number-theoretic settings, see for instance Chapters 3 and 4 of [10]. However, this is not the main viewpoint that we want to consider in this paper.

We want to highlight here the idea that the category of sets is not a good category in which to formulate a notion of points for noncommutative spaces. There are many instances in modern mathematics where it is clear that categories are a natural replacement for sets. Thus, we consider the possibility of a functor of points with values in the category of small categories.

3.1. Bimodules, 2-categories, and a functor of points. Again we fix a field $\mathbb{K}$, which we take to be a number field. As a category of algebras over $\mathbb{K}$ we consider the following.

Definition 3.1. Let $\mathcal{N} \mathcal{A}_{\mathbb{K}}$ denote the category with objects given by associative (not necessarily commutative) algebras $R$ over $\mathbb{K}$ and morphisms $\operatorname{Hom}_{\mathcal{N} \mathcal{A}_{\mathbb{K}}}(R, S)$ given by bimodules ${ }_{R} E_{S}$. The composition of morphisms is given by the tensor product ${ }_{R} E_{S} \otimes_{S} F_{T}=:{ }_{R} F \circ E_{T}$.

The associativity of the tensor product of bimodules can be seen in the following way. Given $E_{1}, E_{2}, E_{3}$, with $E_{i}$ an $R_{i-1}-R_{i}$ module, the tensor product $E=$ $E_{1} \otimes_{R_{1}} E_{2} \otimes_{R_{2}} E_{3}$ is an $R_{0}-R_{3}$ bimodule with a multilinear map $\mu: E_{1} \times E_{2} \times E_{3} \rightarrow E$ from the underlying product of sets that is $\mathbb{K}$-linear in each variable and satisfies $a_{0} \mu\left(e_{1}, e_{2}, e_{3}\right)=\mu\left(a_{0} e_{1}, e_{2}, e_{3}\right), \mu\left(e_{1}, e_{2}, e_{3}\right) a_{3}=\mu\left(e_{1}, e_{2}, e_{3} a_{3}\right), \mu\left(e_{1} a_{1}, e_{2}, e_{3}\right)=$ $\mu\left(e_{1}, a_{1} e_{2}, e_{3}\right), \mu\left(e_{1}, e_{2} a_{2}, e_{3}\right)=\mu\left(e_{1}, e_{2}, a_{2} e_{3}\right)$, for all $a_{i} \in R_{i}$ and $e_{i} \in E_{i}$. The map $\mu$ determines uniquely maps $\mu_{1}:\left(E_{1} \otimes_{R_{1}} E_{2}\right) \times E_{3} \rightarrow E$ and $\mu_{2}: E_{1} \times\left(E_{2} \otimes_{R_{2}} E_{3}\right) \rightarrow E$ and is in turn uniquely determined by each of them.

Remark 3.2. Since $R-S$ bimodules form a category, the $\operatorname{Hom}_{\mathcal{N} \mathcal{A}_{\mathbb{K}}}(R, S)$ are categories, hence $\mathcal{N} \mathcal{A}_{\mathbb{K}}$ is a category enriched over categories, that is, a strict 2-category. 
We then propose the following definition of functor of points for noncommutative spaces. Let Cat denote the category of small categories. This is also a 2-category with objects that are small categories, morphisms that are functors, and 2-morphisms that are natural transformations.

Lemma 3.3. For $R$ an associative (noncommutative) algebra over $\mathbb{K}$, let $\pi_{R}: \mathcal{N} \mathcal{A}_{\mathbb{K}} \rightarrow$ Cat be the functor that assigns to an object $S$ in $\mathcal{N} \mathcal{A}_{\mathbb{K}}$ the category ${ }_{R} \mathcal{B}_{S}$ of $R-S$ bimodules. A morphism ${ }_{S_{1}} F_{S_{2}} \in \operatorname{Hom}_{\mathcal{N} \mathcal{A}_{\mathbb{K}}}\left(S_{1}, S_{2}\right)$ is mapped to the functor $F:{ }_{R} \mathcal{B}_{S_{1}} \rightarrow$ ${ }_{R} \mathcal{B}_{S_{2}}$ that sends ${ }_{R} E_{S_{1}} \mapsto{ }_{R} E_{S_{1}} \otimes_{S_{1} S_{1}} F_{S_{2}}$.

Proof. Recall that in order for $\pi_{R}: \mathcal{N} \mathcal{A}_{\mathbb{K}} \rightarrow$ Cat to be a 2 -functor, it must be an assignment of 0 -cells (objects), 1-cells, and 2-cells in $\mathcal{N} \mathcal{A}_{\mathbb{K}}$ to those in Cat that strictly preserves identity 1 -cells, identity 2 -cells, vertical compositions of 2 -cells, and horizontal compositions of 1-cells and of 2-cells [21].

- For 0-cells $S$ in $\mathcal{N} \mathcal{A}_{\mathbb{K}}, \pi_{R}$ assigns the category ${ }_{R} \mathcal{B}_{S}$ of $R-S$ bimodules;

- For 1-cells $S_{S_{1}} F_{S_{2}} \in \operatorname{Hom}_{\mathcal{N} \mathcal{A}_{\mathbb{K}}}\left(S_{1}, S_{2}\right), \pi_{R}$ assigns the functor $F:{ }_{R} \mathcal{B}_{S_{1}} \rightarrow{ }_{R} \mathcal{B}_{S_{2}}$ that sends ${ }_{R} E_{S_{1}} \mapsto{ }_{R} E_{S_{1}} \otimes_{S_{1} S_{1}} F_{S_{2}}$

- For 2-cells $f:{ }_{S_{1}} F_{S_{2}} \rightarrow{ }_{S_{1}} F_{S_{2}}^{\prime}$ in $\mathcal{N} \mathcal{A}_{\mathbb{K}}, \pi_{R}$ assigns the following natural transformation $\eta: F \rightarrow F^{\prime}$, defined for any ${ }_{R} E_{S_{1}} \in{ }_{R} \mathcal{B}_{S_{1}}, e_{i} \in{ }_{R} E_{S_{1}}$ and $m_{i} \in{ }_{S_{1}} F_{S_{2}}$

$$
\eta_{R} E_{S_{1}}\left(\sum_{i} e_{i} \otimes m_{i}\right)=\sum_{i} e_{i} \otimes f\left(m_{i}\right)
$$

This is a natural transformation since for any bimodule homomorphism $g$ : ${ }_{R} E_{S_{1}} \rightarrow{ }_{R} E_{S_{1}}^{\prime}$

$$
\begin{aligned}
F^{\prime}(g) \circ \eta_{R} E_{S_{1}}\left(\sum_{i} e_{i} \otimes m_{i}\right) & =F^{\prime}(g)\left(\sum_{i} e_{i} \otimes f\left(m_{i}\right)\right) \\
& =\sum_{i} g\left(e_{i}\right) \otimes f\left(m_{i}\right) \\
& =\eta_{R} E_{S_{1}}^{\prime}\left(\sum_{i} g\left(e_{i}\right) \otimes m_{i}\right) \\
& =\eta_{R} E_{S_{1}}^{\prime} \circ F(g)\left(\sum_{i} e_{i} \otimes m_{i}\right)
\end{aligned}
$$

- $\pi_{R}$ preserves identity 1-cells since for any 0-cell $S$ in $\mathcal{N} \mathcal{A}_{\mathbb{K}}, \pi_{R}$ takes its identity 1-cell $S_{S} S_{S}$ to the functor $S:{ }_{R} \mathcal{B}_{S} \rightarrow{ }_{R} \mathcal{B}_{S}$ that sends ${ }_{R} E_{S} \mapsto{ }_{R} E_{S} \otimes_{S} S_{S}$. Since ${ }_{R} E_{S} \otimes_{S} S_{S}={ }_{R} E_{S}$, the functor $S:{ }_{R} \mathcal{B}_{S} \rightarrow{ }_{R} \mathcal{B}_{S}$ is the identity functor of ${ }_{R} \mathcal{B}_{S}$

- $\pi_{R}$ preserves identity 2-cells since any identity bimodule homomorphism $f$ : ${ }_{S_{1}} F_{S_{2}} \rightarrow{ }_{S_{1}} F_{S_{2}}$ is sent to the natural transformation $\eta: F \rightarrow F$, defined for 
any ${ }_{R} E_{S_{1}} \in{ }_{R} \mathcal{B}_{S_{1}}, e_{i} \in{ }_{R} E_{S_{1}}$ and $m_{i} \in{ }_{S_{1}} F_{S_{2}}$

$$
\begin{aligned}
\eta_{R} E_{S_{1}}\left(\sum_{i} e_{i} \otimes m_{i}\right) & =\sum_{i} e_{i} \otimes f\left(m_{i}\right) \\
& =\sum_{i} e_{i} \otimes m_{i}
\end{aligned}
$$

which is precisely an identity natural transformation since it maps each object ${ }_{R} E_{S_{1}}$ of ${ }_{R} \mathcal{B}_{S_{1}}$ to the identity morphism id $\left.{ }_{F(}{ }_{R} E_{S_{1}}\right)$ in ${ }_{R} \mathcal{B}_{S_{2}}$

- For any two composable bimodules ${ }_{S_{1}} F_{S_{2}}, S_{2} G_{S_{3}}$,

$$
\begin{aligned}
\pi_{R}\left(S_{1} F \circ G_{S_{3}}\right) & =\pi_{R}\left({ }_{S_{1}} F_{S_{2}} \otimes_{S_{2} S_{2}} G_{S_{3}}\right) \\
& =-\otimes_{S_{1} S_{1}} F_{S_{2}} \otimes_{S_{2} S_{2}} G_{S_{3}} \\
& =\left(-\otimes_{S_{1} S_{1}} F_{S_{2}}\right) \circ\left(-\otimes_{S_{2} S_{2}} G_{S_{3}}\right) \\
& =\pi_{R}\left({ }_{S_{1}} F_{S_{2}}\right) \circ \pi_{R}\left({ }_{S_{2}} G_{S_{3}}\right)
\end{aligned}
$$

showing that $\pi_{R}$ preserves the horizontal compositions of 1-cells;

- For two vertically composable bimodule homomorphisms $f:{ }_{S_{1}} F_{S_{2}} \rightarrow{ }_{S_{1}} G_{S_{2}}$ and $g:{ }_{S_{1}} G_{S_{2}} \rightarrow{ }_{S_{1}} H_{S_{2}}$ in $\mathcal{N} \mathcal{A}_{\mathbb{K}}$, and ${ }_{R} E_{S_{1}} \in{ }_{R} \mathcal{B}_{S_{1}}, e_{i} \in{ }_{R} E_{S_{1}}$ and $m_{i} \in{ }_{S_{1}} F_{S_{2}}$

$$
\begin{aligned}
\pi_{R}(g \circ f)_{R} E_{S_{1}}\left(\sum_{i} e_{i} \otimes m_{i}\right) & =\sum_{i} e_{i} \otimes(g \circ f)\left(m_{i}\right) \\
& =\pi_{R}(g)_{R} E_{S_{1}}\left(\sum_{i} e_{i} \otimes f\left(m_{i}\right)\right) \\
& =\left(\pi_{R}(g) \circ \pi_{R}(f)\right)_{R} E_{S_{1}}\left(\sum_{i} e_{i} \otimes m_{i}\right)
\end{aligned}
$$

- Finally, for two horizontally composable bimodule homomorphisms $f:{ }_{S_{1}} F_{S_{2}} \rightarrow$ ${ }_{S_{1}} F_{S_{2}}^{\prime}$ and $g:{ }_{S_{2}} G_{S_{3}} \rightarrow{ }_{S_{2}} G_{S_{3}}^{\prime}$, and ${ }_{R} E_{S_{1}} \in{ }_{R} \mathcal{B}_{S_{1}}, e_{i} \in{ }_{R} E_{S_{1}}, m_{i} \in{ }_{S_{1}} F_{S_{2}}$, $n_{i} \in S_{2} G_{S_{3}}$,

$$
\begin{aligned}
\pi_{R}(f \otimes g)_{R} E_{S_{1}}\left(\sum_{i} e_{i} \otimes m_{i} \otimes n_{i}\right) & =\sum_{i} e_{i} \otimes f\left(m_{i}\right) \otimes g\left(n_{i}\right) \\
& =\pi_{R}(f)_{R} E_{S_{1}}\left(\sum_{i} e_{i} \otimes m_{i} \otimes g\left(n_{i}\right)\right) \\
& =\left(\pi_{R}(f) \circ \pi_{R}(g)\right)_{R} E_{S_{1}}\left(\sum_{i} e_{i} \otimes m_{i} \otimes n_{i}\right)
\end{aligned}
$$

The previous result motivates the following definition.

Definition 3.4. Let $X_{R}^{n c}$ denote the noncommutative space determined by the algebra $R$ over $\mathbb{K}$. We define the noncommutative functor of points by setting $X_{R}^{n c}(S):={ }_{R} \mathcal{B}_{S}$ for any $\mathbb{K}$-algebra $S$.

Thus, the $S$-points $X_{R}^{n c}(S)$ of the noncommutative space $X_{R}^{n c}$ form a 2-category rather than a set and consist of all $R-S$ bimodules. 
Thus, we consider $X_{R}^{n c}(S)={ }_{R} \mathcal{B}_{S}$ to be our kinematic space for noncommutative sigma models, where the noncommutative space $X_{R}^{n c}$ is the (fixed) target space of the sigma model and the noncommutative space determined by the algebra $S$ is the (variable) source space of the sigma model.

The next step is then to make these spaces dynamical by assigning an action functional that generalizes the height action functional we have been discussing in the previous section.

\section{The CASE of Finite Dimensional AlgebRas}

Before discussing the general problem of how to obtain an action functional on on our noncommutative "functor of points" $X_{R}^{n c}(S)={ }_{R} \mathcal{B}_{S}$, we focus on the simpler case of finite dimensional algebras.

It is convenient to adopt the viewpoint of Arakelov geometry in a noncommutative setting, [6], 7]. Consider a finite dimensional semisimple algebras $A$ over a number field $\mathbb{K}$. In the case where $A$ is a division algebra, the construction of a height function for free submodules of $A^{n}$ was obtained in [32], generalizing the commutative construction of heights of subspaces of a vector space over a number field using volumes of Euclidean lattices (see [53]). If $A$ is not a division algebra, then it has zero-divisors, and this prevents the usual construction of valuations. This case is analyzed in [6], where a different way of defining a height function $h_{\mathcal{O}}(V)$ of a free submodule of $A^{n}$ is introduced, which for division algebras agrees up to a scale factor with the height defined in 32 .

The general structure of finite dimensional algebras is analyzed in [17]. For our purposes, we consider the case of semi-simple algebras, since these algebras can be viewed as sums of simple algebras, and this naturally generalizes to the number field case the much simpler complex case where one deals with sums of matrix algebras. Indeed, every simple algebra is isomorphic to a matrix algebra $M_{n}(D)$ over some division algebra $D$ over $\mathbb{K}$.

We show here how to adapt in our setting this approach to associate an action functional defined on our $X_{R}^{n c}(S)={ }_{R} \mathcal{B}_{S}$, in the case where both $R$ and $S$ are semisimple finite dimensional algebras over a number field $\mathbb{K}$.

4.1. Noncommutative arithmetic curves. In arithmetic geometry, an arithmetic (affine) curve is $\operatorname{Spec}\left(\mathcal{O}_{\mathbb{K}}\right)$, for a number field $\mathbb{K}$ with ring of integers $\mathcal{O}_{\mathbb{K}}$. The curve is "compactified" by adding to $\operatorname{Spec}\left(\mathcal{O}_{\mathbb{K}}\right)$ the "primes at infinity", that is, the $n$ embeddings $\sigma: \mathbb{K} \hookrightarrow \mathbb{C}$ for $n=[\mathbb{K}: \mathbb{Q}]=\operatorname{deg}(\mathbb{K})$ the degree of the number field. Heuristically, one thinks of an arithmetic curve $\operatorname{Spec}\left(\mathcal{O}_{\mathbb{K}}\right)$ as having at each finite place $\wp$ in $\operatorname{Spec}\left(\mathcal{O}_{\mathbb{K}}\right)$ a copy of the corresponding residue field $\mathbb{F}_{q}=\mathcal{O}_{\mathbb{K}} / \wp$. (At the archimedean places these should be replaced by a more mysterious object $\mathbb{F}_{1}$, which has many different mathematical incarnations, [34, 39].)

In noncommutative algebraic geometry there is a similar notion of an arithmetic noncommutative curve, see [6], which we review briefly here. 
Definition 4.1. Given a semisimple finite dimensional algebra $A$ over a number field $\mathbb{K}$, an $\mathcal{O}_{\mathbb{K}^{-}}$-order $\mathcal{O}_{A}$ in $A$ is a subring of $A$ such that $\mathcal{O}_{A}$ is a full $\mathcal{O}_{\mathbb{K}}$-lattice in $A$.

This means that $\mathcal{O}_{A}$ is a finitely generated torsion free $\mathcal{O}_{\mathbb{K}}$-module with the property that $\mathbb{K} \mathcal{O}_{A}=A$ as $\mathbb{K}$-vector spaces.

A notion of arithmetic noncommutative curve is then provided by $\operatorname{Spec}\left(\mathcal{O}_{A}\right)$ where $\mathcal{O}_{A}$ is an $\mathcal{O}_{\mathbb{K}^{-}}$order in $A$. The reason why it makes sense to consider this a noncommutative arithmetic curve lies in the fact that the prime ideals $\wp$ in $\operatorname{Spec}\left(\mathcal{O}_{A}\right)$ are maximal two-sided ideas of $\mathcal{O}_{A}$ and the quotients $\mathcal{O}_{A} / \wp$ are simple algebras hence isomorphic to some matrix algebra $M_{k_{\wp}}\left(D_{\wp}\right)$ over some division algebra $D_{\wp}$. Thus, this construction replaces the commutative objects $\mathbb{F}_{q}$ attached to the places $\wp$ of $\operatorname{Spec}\left(\mathcal{O}_{\mathbb{K}}\right)$ in a classical (commutative) arithmetic curve, with the noncommutative objects $M_{k_{\wp}}\left(D_{\wp}\right)$ attached to the places $\wp$ of the noncommutative curve $\operatorname{Spec}\left(\mathcal{O}_{A}\right)$.

While this point of view is very helpful, it is slightly different from the one we will be following here, since it is still based on taking two-sided ideals $\wp$, related to a classical notion of points. We will however, keep this main idea in mind in framing our setting, while adapting it to our notion of points described in the previous section.

Before developing our setting, we recall a few more aspects of the theory of noncommutative arithmetic curves, as developed by Borek in [6], which will be useful in our setting as well, in particular some notions of height that we will be adapting to our setting in the next subsections.

For a semisimple finite dimensional algebra $A$ over a number field $\mathbb{K}$, We write $A_{\mathbb{R}}:=A \otimes_{\mathbb{K}} \mathbb{R}$ and $A_{\mathbb{R}}^{*}:=\mathrm{GL}_{1}\left(A_{\mathbb{R}}\right)$ for the group of units (multiplicatively invertible elements) in $A_{\mathbb{R}}$. For a $\mathcal{O}_{\mathbb{K}}$-order $\mathcal{O}_{A}$ in $A$, we also write $\mathcal{J}\left(\mathcal{O}_{A}\right)$ for the set of full left- $\mathcal{O}_{A}$-ideals in $A$. These are left $\mathcal{O}_{A}$-modules that are full $\mathcal{O}_{\mathbb{K}}$-lattices. For a full left- $\mathcal{O}_{A}$-ideal $\mathfrak{a}$ in $A$ there is an $r \in \mathcal{O}_{\mathbb{K}}$ such that $\mathfrak{a} r \subset \mathcal{O}_{A}$ is a left-ideal in $\mathcal{O}_{A}$. The set of complete $\mathcal{O}_{A}$-ideals is $\hat{\mathcal{J}}\left(\mathcal{O}_{A}\right)=\mathcal{J}\left(\mathcal{O}_{A}\right) \times A_{\mathbb{R}}^{*}$. We write complete $\mathcal{O}_{A}$-ideals as $\overline{\mathfrak{a}}=\left(\mathfrak{a}, \mathfrak{a}_{\infty}\right) \in \mathcal{J}\left(\mathcal{O}_{A}\right) \times A_{\mathbb{R}}^{*}$, as in [6]. For $\mathfrak{a} \in \mathcal{J}\left(\mathcal{O}_{A}\right)$ one takes

$$
\mathcal{N}(\mathfrak{a})=\frac{\#\left(\mathcal{O}_{A} / \mathfrak{a} r\right)}{\#\left(\mathcal{O}_{A} / \mathcal{O}_{A} r\right)}
$$

This is independent of the choice of an $r \in \mathcal{O}_{\mathbb{K}}$ for which $\mathfrak{a} r$ is a left-ideal in $\mathcal{O}_{A}$. As shown in Theorem 1 of [6], the norm $\mathcal{N}(\mathfrak{a})$ of (4.1) has an equivalent expression as product over the prime ideals $\wp$ in $\operatorname{Spec}\left(\mathcal{O}_{A}\right)$. The "absolute norm" $\mathcal{N}(\overline{\mathfrak{a}})$ is given by the product of $\mathcal{N}(\mathfrak{a})$ and a contribution of the archimedean component $\mathfrak{a}_{\infty}$ given by $\left|N_{A_{\mathbb{R}} \mid \mathbb{R}}\left(\mathfrak{a}_{\infty}\right)\right|$ where $N_{A_{\mathbb{R}} \mid \mathbb{R}}$ is the norm map from $A_{\mathbb{R}}$ to $\mathbb{R}$. (In general, given a finite dimensional algebra $B$ over a field $F$ an element $x \in B$ acts by left multiplication defining an $F$-endomorphism of $B$. The norm $N_{B \mid F}: B \rightarrow F$ is the multiplicative map given by $N_{B \mid F}(x)=\operatorname{det}(x)$, the determinant of the resulting $F$-linear transformation.)

One can also define a volume $\operatorname{vol}(\overline{\mathfrak{a}}):=\operatorname{vol}\left(\mathfrak{a}_{\infty} j(\mathfrak{a})\right)$, where $j: A \rightarrow A_{\mathbb{R}}$ maps $\mathfrak{a}$ to a $\mathbb{Z}$-lattice $j(\mathfrak{a})$ in $A_{\mathbb{R}}$ with $\mathbb{R} j(\mathfrak{a})=A_{\mathbb{R}}$, and the volume of the lattice $\mathfrak{a}_{\infty} j(\mathfrak{a})$ is the volume of its fundamental domain in the measure defined by the norm associated to the bilinear form $A_{\mathbb{R}} \times A_{\mathbb{R}} \rightarrow \mathbb{R}$ determined by the trace $(x, y) \mapsto \operatorname{Tr}_{A_{\mathbb{R}} \mathbb{R}}(x y)$. This is related to the absolute norm by $\operatorname{vol}(\overline{\mathfrak{a}})=\mathcal{N}(\overline{\mathfrak{a}}) \operatorname{vol}\left(\mathcal{O}_{A}\right)$, see $\S 4$ of [6]. We will discuss 
more generally in $\$ 4.7$ and 4.8 the assignment of a volume to a normed space and notions of volume we will be using in the construction of height functions.

The last notions that we need to recall from the Arakelov geometry of noncommutative curves of [6] is arithmetic vector bundles and the associated arithmetic degree map. Again, the notion of arithmetic vector bundle that we will be using is slightly different from the one used in [6] that we recall here, but it is closely related.

Definition 4.2. Let $\mathbb{K}$ be a number field and $A$ a semisimple finite dimensional algebra over $\mathbb{K}$, with $\mathcal{O}_{A}$ an order in $A$. Let $\mathcal{E}$ be a left $\mathcal{O}_{A}$-module. Let $\sigma$ be an archimedean place of $\mathbb{K}$ and let $\mathbb{K}_{\sigma}$ be either $\mathbb{R}$ or $\mathbb{C}$, with the corresponding embedding $\sigma: \mathbb{K} \hookrightarrow \mathbb{K}_{\sigma}$, and let $A_{\sigma}=\mathbb{K}_{\sigma} \otimes_{\mathbb{K}} A$ and $\mathcal{E}_{\sigma}=A_{\sigma} \otimes_{\mathcal{O}_{A}} \mathcal{E}$. A hermitian structure $h$ on $\mathcal{E}$ is a $\star$-hermitian bilinear form $h: \mathcal{E}_{\sigma} \times \mathcal{E}_{\sigma} \rightarrow A_{\sigma}$ such that $\operatorname{Tr}_{A_{\sigma} \mid \mathbb{K}_{\sigma}} \circ h$ : $\mathcal{E}_{\sigma} \times \mathcal{E}_{\sigma} \rightarrow \mathbb{K}_{\sigma},(x, y) \mapsto \operatorname{Tr}_{A_{\sigma} \mid \mathbb{K}_{\sigma}}(h(x, y))$ is positive definite.

Such hermitian metrics can be constructed in the following way, [6]. The verification of the following statement is immediate and we omit it.

Lemma 4.3. The choice of a collection of elements $\beta_{i} \in A_{\sigma}^{*}$, for $i=1, \ldots, n$ determines a hermitian structure on the free module $A_{\sigma}^{n}$ by setting $h(x, y)=\sum_{i} x_{i} \beta_{i} \beta_{i}^{*} y_{i}^{*}$. $B y$ restriction, it determines a hermitian structure on a projective $A_{\sigma}$-module that is a summand of the free module $A_{\sigma}^{n}$.

In more physical terms, we can regard $\rho_{i}=\beta_{i} \beta_{i}^{*}$ as a "density matrix" and $\operatorname{Tr}_{A_{\sigma} \mid \mathbb{K}_{\sigma}}$ ○ $h(x, y)=\operatorname{Tr}\left(y^{*} x \rho\right)$ as the state associated to this density matrix.

Definition 4.4. Let $\mathcal{E}$ be a finite projective left $A$-module. For $\sigma$ an archimedean place of $\mathbb{K}$, a hermitian structure $h: \mathcal{E}_{\sigma} \times \mathcal{E}_{\sigma} \rightarrow \mathbb{K}_{\sigma}$ is said to be "standard" if it is the restriction of a hermitian form as in Lemma 4.3 on $A_{\sigma}^{n}$, for an embedding $j_{A}: \mathcal{E}_{\sigma} \hookrightarrow A_{\sigma}^{n}$ realizing $\mathcal{E}_{\sigma}$ as a summand of a free module.

It is shown in [6] that all hermitian structures $h: A_{\mathbb{R}} \times A_{\mathbb{R}} \rightarrow A_{\mathbb{R}}$ on a real semisimple finite dimensional algebra are of the standard form $h:(x, y) \mapsto x \beta \beta^{*} y^{*}$ as above. The standard hermitian structures will be sufficient for our purposes, so we will limit some of the arguments to this case.

An arithmetic vector bundle over the noncommutative arithmetic curve $\operatorname{Spec}\left(\mathcal{O}_{A}\right)$ is defined in [6] as a pair $(\mathcal{E}, h)$, where $\mathcal{E}$ is a left $\mathcal{O}_{A}$-module that is also an $\mathcal{O}_{\mathbb{K}}$-lattice, such that $A \otimes_{\mathcal{O}_{A}} \mathcal{E}$ is a free $A$-module, and $h$ is a hermitian structure $h: \mathcal{E}_{\mathbb{R}} \times \mathcal{E}_{\mathbb{R}} \rightarrow$ $A_{\mathbb{R}}$ on $\mathcal{E}_{\mathbb{R}}=A_{\mathbb{R}} \otimes_{\mathcal{O}_{A}} \mathcal{E}$. Our notion of arithmetic vector bundle is discussed in Definition 4.5 below.

The Grothendieck group $K_{0}\left(\operatorname{Spec}\left(\mathcal{O}_{A}\right)\right)$ of arithmetic vector bundles of [6] over the noncommutative arithmetic curve $\operatorname{Spec}\left(\mathcal{O}_{A}\right)$ is generated by isomorphism classes of $(\mathcal{E}, h)$, where isomorphism is given by isomorphisms of left $\mathcal{O}_{A^{-}}$-modules that preserve the hermitian structure, modulo the relations $[(\mathcal{E}, h)]=\left[\left(\mathcal{E}^{\prime}, h^{\prime}\right)\right]+\left[\left(\mathcal{E}^{\prime \prime}, h^{\prime \prime}\right)\right]$ for short exact sequences $0 \rightarrow \mathcal{E}^{\prime} \rightarrow \mathcal{E} \rightarrow \mathcal{E}^{\prime \prime} \rightarrow 0$ of $\mathcal{O}_{A}$-modules such that the sequence $0 \rightarrow \mathcal{E}_{\mathbb{R}}^{\prime} \rightarrow \mathcal{E}_{\mathbb{R}} \rightarrow \mathcal{E}_{\mathbb{R}}^{\prime \prime} \rightarrow 0$ splits orthogonally, that is, with $\mathcal{E}_{\mathbb{R}}^{\prime \prime}=\left(\mathcal{E}_{\mathbb{R}}^{\prime}\right)^{\perp}$ the orthogonal with respect to the hermitian structure. 
Complete $\mathcal{O}_{A}$-ideals $\overline{\mathfrak{a}} \in \hat{\mathcal{J}}\left(\mathcal{O}_{A}\right)$ determine hermitian line bundles $\mathcal{L}(\overline{\mathfrak{a}})$ that generate the Grothendieck group $K_{0}\left(\operatorname{Spec}\left(\mathcal{O}_{A}\right)\right)$. The degree map

$$
\operatorname{deg}_{\mathcal{O}_{A}}(\mathcal{L}(\overline{\mathfrak{a}})):=-\log \mathcal{N}(\overline{\mathfrak{a}})
$$

extends uniquely to a degree map on $K_{0}\left(\operatorname{Spec}\left(\mathcal{O}_{A}\right)\right)$.

Finally, we recall the notion of height used in the Arakelov geometry of noncommutative curves, [6]. Let $h_{n}$ denote the hermitian metric on $A_{\mathbb{R}}^{n}$ given by $h_{n}$ : $\left(\left(x_{1}, \ldots, x_{N}\right),\left(y_{1}, \ldots, y_{N}\right)\right) \mapsto \sum_{i} x_{i}^{*} y_{i}$. For a free $A$-submodule $\mathcal{V}$ of $A^{n}$ the logarithmic height is defined as

$$
h_{\mathcal{O}_{A}}(\mathcal{V})=\log H_{\mathcal{O}_{A}}(\mathcal{V}):=-\operatorname{deg}_{\mathcal{O}_{A}}\left(\mathcal{V} \cap \mathcal{O}_{A}^{n}, h_{n}\right)
$$

By the relation of the norm to the volume, the height can be further identified with

$$
H_{\mathcal{O}_{A}}(\mathcal{V})=\frac{\operatorname{vol}\left(\mathcal{V} \cap \mathcal{O}_{A}^{n}\right)}{\operatorname{vol}\left(\mathcal{O}_{A}\right)^{\operatorname{rank}(\mathcal{V})}},
$$

where the volume in $A_{\mathbb{R}}^{n}$ is defined by the norm associated to the quadratic form $\operatorname{Tr}_{A_{\mathbb{R}} \mid \mathbb{R}} \circ h_{n}$. This generalizes to the noncommutative setting the height of [53]. In the case of division algebras it agrees (up to scaling factor given by the degree $[\mathbb{K}: \mathbb{Q}]$ ) with the height of [31], [32], 33]. Here the hypotheses that an arithmetic vector bundle is a free $A$-module as well as an $\mathcal{O}_{\mathbb{K}}$-lattice, assumed in [6] are used in defining the volume of $\mathcal{V} \cap \mathcal{O}_{A}^{n}$ and the rank of $\mathcal{V}$. We will discuss how this definition of height adapts to our setting in the next subsections.

4.2. Free modules versus finite projective modules. The notion of arithmetic vector bundle over a noncommutative arithmetic curve, that we recalled above from [6], includes the requirement that the left $\mathcal{O}_{A}$-module $\mathcal{E}$ satisfies the property that $A \otimes_{\mathcal{O}_{A}} \mathcal{E}$ is a free $A$-module.

It would seem more natural, if we want to regard $\mathcal{E}$ as a vector bundle, to relax this requirement and only require that $A \otimes_{\mathcal{O}_{A}} \mathcal{E}$ is a finite projective $A$-module. As we see in the following, in our setting based on bimodules, this less restrictive condition will allow us to include some very natural families of bimodules that we certainly want to include in our counting of "points" over our noncommutative arithmetic spaces.

Thus, in our setting, we give the following slightly different definition of an arithmetic vector bundle.

Definition 4.5. Let $A$ be a semisimple finite dimensional algebra over a number field $\mathbb{K}$ and let $\mathcal{O}_{A}$ be an order in $A$. A left arithmetic $\left(A, \mathcal{O}_{A}\right)$-vector bundle $(\mathcal{E}, h)$ is a left $\mathcal{O}_{A}$-module, together with the assignment $h=\left(h_{\sigma}\right)$, for all archimedean places $\sigma$ of $\mathbb{K}$, of a hermitian structure $h: \mathcal{E}_{\sigma} \times \mathcal{E}_{\sigma} \rightarrow A_{\sigma}$.

Since $A$ is a semisimple finite dimensional algebra, the $A$-module $A \otimes_{\mathcal{O}_{\mathbb{K}}} \mathcal{E}$ is finite projective (see also Remark 4.8 below). 
4.3. Functor of points on finite dimensional algebras. We now adapt the notions developed in the Arakelov geometry of noncommutative arithmetic curves in [6] to our setting.

Let $A$ be a semisimple finite dimensional algebra over a number field $\mathbb{K}$. Let $\sigma: \mathbb{K} \hookrightarrow \mathbb{C}$ be an embedding (either real or complex), that is, an archimedean place of $\mathbb{K}$. We can associate to $A$ and $\sigma$ a real or complex algebra $A_{\sigma}=A \otimes_{\mathbb{K}, \sigma} \mathbb{R}$ for a real embedding and $A_{\sigma}=A \otimes_{\mathbb{K}, \sigma} \mathbb{C}$ for a complex embedding. Assuming $A=\oplus_{i} M_{n_{i}}\left(D_{i}\right)$ where the simple summands are matrix algebras of rank $n_{i}$ over a division algebra $D_{i}$ over $\mathbb{K}$, we can similarly decompose $A_{\sigma}=\oplus_{i} M_{m_{i}}(\mathbb{C})$ in the complex case and $A_{\sigma}=\oplus_{i} M_{r_{i}}(\mathbb{R}) \oplus_{j} M_{r_{j}}(\mathbb{C}) \oplus_{k} M_{r_{k}}(\mathbb{H})$ for the real case, where $\mathbb{H}$ is the division algebra of quaternions.

Definition 4.6. An arithmetic structure on a semisimple finite dimensional algebra $A$ over a number field $\mathbb{K}$ is the choice of an $\mathcal{O}_{\mathbb{K}}$-order $\mathcal{O}_{A}$ in $A$. Given two such a choices $\left(A, \mathcal{O}_{A}\right)$ and $\left(B, \mathcal{O}_{B}\right)$, we define the category of arithmetic hermitian bimodules ${ }_{\left(A, \mathcal{O}_{A}\right)} \mathcal{H}_{\left(B, \mathcal{O}_{B}\right)}$ with objects the pairs $(\mathcal{E}, h)$ where $\mathcal{E}$ is an $\mathcal{O}_{A}-\mathcal{O}_{B}$ bimodule, such that $A \otimes_{\mathcal{O}_{A}} \mathcal{E}$ and $\mathcal{E} \otimes_{\mathcal{O}_{B}} B$ are finite projective as $A$-module and $B$-module, respectively. Moreover, for any archimedean place $\sigma$ of $\mathbb{K}$ the bimodule $\mathcal{E}_{\sigma}:=A_{\sigma} \otimes_{\mathcal{O}_{A}} \mathcal{E} \otimes_{\mathcal{O}_{B}} B_{\sigma}$ is endowed with a pair of hermitian structures $h=\left(h_{A}, h_{B}\right)$ with $h_{A}: \mathcal{E}_{\sigma} \times \mathcal{E}_{\sigma} \rightarrow A_{\sigma}$ and $h_{B}: \mathcal{E}_{\sigma} \times \mathcal{E}_{\sigma} \rightarrow B_{\sigma}$ with the following properties

- For all $a \in A_{\sigma}$, the identities $h_{A}(a x, y)=a h_{A}(x, y)$ and $h_{A}(x, a y)=h_{A}(x, y) a^{*}$ hold for all $(x, y) \in \mathcal{E}_{\sigma} \times \mathcal{E}_{\sigma}$.

- For all $x \in \mathcal{E}_{\sigma}$ the element $h_{A}(x, x)$ is a positive element in $A_{\sigma}$ (that is, an element of the form $\beta^{*} \beta$ for some $\beta \in A_{\sigma}$ and $h_{A}(x, x)=0$ iff $x=0$.

- For all $b \in B_{\sigma}$, the identities $h_{B}(x, y b)=h_{B}(x, y) b$ and $h_{B}(x b, y)=b^{*} h_{B}(x, y)$ hold for all $(x, y) \in \mathcal{E}_{\sigma} \times \mathcal{E}_{\sigma}$.

- For all $x \in \mathcal{E}_{\sigma}$ the element $h_{B}(x, x)$ is a positive element in $B_{\sigma}$ and $h_{B}(x, x)=0$ iff $x=0$.

Morphisms $\phi:\left(\mathcal{E}, h_{A}, h_{B}\right) \rightarrow\left(\mathcal{E}^{\prime}, h_{A}^{\prime}, h_{B}^{\prime}\right)$ are morphisms $\phi: \mathcal{E} \rightarrow \mathcal{E}^{\prime}$ of $\mathcal{O}_{A}-\mathcal{O}_{B}$ bimodules such that the induced morphisms $\phi_{\sigma}: \mathcal{E}_{\sigma} \rightarrow \mathcal{E}_{\sigma}^{\prime}$ satisfy $h_{A}^{\prime}\left(\phi_{\sigma}(x), \phi_{\sigma}(y)\right)=$ $h_{A}(x, y)$ and $h_{B}^{\prime}\left(\phi_{\sigma}(x), \phi_{\sigma}(y)\right)=h_{B}(x, y)$.

Definition 4.7. Consider an arithmetic structure on a semisimple finite dimensional algebra $A$ over a number field $\mathbb{K}$ as in Definition 4.6. A strong arithmetic hermitian bimodule $(\mathcal{E}, h)$ is an object of ${ }_{\left(A, \mathcal{O}_{A}\right)} \mathcal{H}_{\left(B, \mathcal{O}_{B}\right)}$ as in Definition 4.6 with the additional properties that $\mathcal{E}$ is also left-right $\mathcal{O}_{\mathbb{K}}$-lattice, such that $A \otimes_{\mathcal{O}_{A}} \mathcal{E}$ is a free $A$-module and $\mathcal{E} \otimes_{\mathcal{O}_{B}} B$ is a free $B$-module.

Definition 4.7 more closely matches the notion of arithmetic vector bundles used in [6]. We prefer here to consider the more general class of bimodules of Definition 4.6.

Remark 4.8. The condition that $A \otimes_{\mathcal{O}_{A}} \mathcal{E}$ and $\mathcal{E} \otimes_{\mathcal{O}_{B}} B$ are finite projective modules in Definition 4.6 is automatically satisfied since $A$ and $B$ are semisimple algebras ([29], Theorem 2.8 and Corollary 3.7). 
Given such a choice of a pair $\left(A, \mathcal{O}_{A}\right)$, and another $\left(B, \mathcal{O}_{B}\right)$, we define as in the previous section the noncommutative functor of points $X_{\left(A, \mathcal{O}_{A}\right)}^{n c}\left(B, \mathcal{O}_{B}\right)$ (the $\left(B, \mathcal{O}_{B}\right)$ "points" of the noncommutative space defined by $\left.\left(A, \mathcal{O}_{A}\right)\right)$ to be the category of hermitian bimodules defined as above,

$$
X_{\left(A, \mathcal{O}_{A}\right)}^{n c}\left(B, \mathcal{O}_{B}\right):={ }_{\left(A, \mathcal{O}_{A}\right)} \mathcal{H}_{\left(B, \mathcal{O}_{B}\right)} .
$$

We refer here to arithmetic vector bundles over $\operatorname{Spec}\left(\mathcal{O}_{A}\right)$ as left arithmetic vector bundles and we similarly define right arithmetic vector bundles $(\mathcal{E}, h)$ in the same way but with $\mathcal{E}$ a right $\mathcal{O}_{A}$-module.

4.4. Hermitian structures on bimodules. We discuss here some explicit construction of hermitian structures on bimodules between semisimple finite dimensional algebras, which will be useful later in the construction of the height function.

Lemma 4.9. As above, let $\left(A, \mathcal{O}_{A}\right)$ and $\left(B, \mathcal{O}_{B}\right)$ be pairs of semisimple finite dimensional algebras over a number field $\mathbb{K}$ and orders. Let $\left(\mathcal{E}_{A}, h_{A}^{\mathcal{E}_{A}}\right)$ be a left arithmetic $\left(A, \mathcal{O}_{A}\right)$-vector bundle and let $\left(\mathcal{E}_{B}, h_{B}^{\mathcal{E}_{B}}\right)$ be a right arithmetic $\left(B, \mathcal{O}_{B}\right)$-vector bundle, where the hermitian structures are standard as in Definition 4.4. Then $\mathcal{E}=\mathcal{E}_{A} \otimes_{\mathbb{K}} \mathcal{E}_{B}$ is an arithmetic hermitian bimodule with standard hermitian structures.

Proof. $\mathcal{E}=\mathcal{E}_{A} \otimes_{\mathbb{K}} \mathcal{E}_{B}$ is a hermitian bimodule if it satisfies all the conditions in Definition 4.6. Given that $\left(\mathcal{E}_{A}, h_{A}\right)$ and $\left(\mathcal{E}_{B}, h_{B}\right)$ are left and right arithmetic vector bundles, respectively, then $\mathcal{E}=\mathcal{E}_{A} \otimes_{\mathbb{K}} \mathcal{E}_{B}$ is an $\mathcal{O}_{A}-\mathcal{O}_{B}$ bimodule. As noted in Remark 4.8, the condition that $A \otimes_{\mathcal{O}_{A}} \mathcal{E}$ and $\mathcal{E} \otimes_{\mathcal{O}_{B}} B$ are finite projective modules is automatically satisfied.

For any archimedean place $\sigma$ of $\mathbb{K}$, one needs to show that the bimodule $\mathcal{E}_{\sigma}:=$ $A_{\sigma} \otimes_{\mathcal{O}_{A}} \mathcal{E} \otimes_{\mathcal{O}_{B}} B_{\sigma}$ can be endowed with a pair of hermitian structures $h=\left(h_{A}, h_{B}\right)$ with $h_{A}: \mathcal{E}_{\sigma} \times \mathcal{E}_{\sigma} \rightarrow A_{\sigma}$ and $h_{B}: \mathcal{E}_{\sigma} \times \mathcal{E}_{\sigma} \rightarrow B_{\sigma}$ satisfying all the conditions in Definition 4.6.

Consider the given hermitian structure $h_{A}^{\mathcal{E}_{A}}: \mathcal{E}_{A, \sigma} \times \mathcal{E}_{A, \sigma} \rightarrow A_{\sigma}$. We are assuming it is standard in the sense of Definition 4.4, hence we have:

- an integer $n=n_{A} \in \mathbb{N}$ such that $\mathcal{E}_{A}=A^{n} e_{A}$ for an idempotent $e_{A} \in M_{n}(A)$

- elements $\beta_{A, i} \in A^{*}$, for $i=1, \ldots, n$ such that $h_{A}^{\mathcal{E}_{A}}(x, y)=\sum_{i} x_{i} \beta_{A, i} \beta_{A, i}^{*} y_{i}=$ : ${ }_{A}\langle x, y\rangle$

- elements $\left\{u_{i}\right\}$ in $\mathcal{E}_{A}$ such that $x=\sum_{i} x_{i} u_{i}$ and $y=\sum_{i} y_{i} u_{i}$ with $x_{i}={ }_{A}\left\langle x, u_{i}\right\rangle$ and $y_{i}={ }_{A}\left\langle y, u_{i}\right\rangle$ and with $\left(e_{A}\right)_{i j}={ }_{A}\left\langle u_{i}, u_{j}\right\rangle$.

Consider $\mathcal{E}_{B}$ as a vector space over $\mathbb{K}$, and for each $i=1, \ldots, n$ let $\left\{v_{i, \ell}\right\}_{\ell \in \mathcal{J}}$ be a choice of a basis for $\mathcal{E}_{B}$ as $\mathbb{K}$-vector space. Then the set $\left\{u_{i} \otimes v_{i, \ell}\right\}$ of elements of $\mathcal{E}_{A} \otimes_{\mathbb{K}} \mathcal{E}_{B}$ has the property that $\xi=\sum_{i, \ell} x_{i, \ell} u_{i} \otimes v_{i, \ell}$, for $x \in \mathcal{E}_{A} \otimes_{\mathbb{K}} \mathcal{E}_{B}$, with the $x_{i, \ell} \in A$.

One can then define $h_{A}$ on $\mathcal{E}_{\sigma}$ as follows. We write an element $x \in \mathcal{E}_{\sigma}$ as above, with the $x_{i, \ell} \in A_{\sigma}$ and similarly for $y=\sum_{i, \ell} y_{i, \ell} u_{i} \otimes v_{i, \ell}$. With a slight abuse of notation we will still denote by $\beta_{A, i}$ as above the diagonal matrix in $M_{r}(A)$ with 
$r=\# J$ with $\beta_{A, i} \in A^{*}$ on the diagonal. We can then define

$$
h_{A}(x, y):=\sum_{i} x_{i} \beta_{A, i} \beta_{A, i}^{*} y_{i}^{*}=\sum_{\ell} h_{A}^{\mathcal{E}_{A}}\left(x_{\ell}, y_{\ell}\right)
$$

where $x_{i} \beta_{A, i} \beta_{A, i}^{*} y_{i}^{*}=\sum_{\ell, \ell^{\prime} \in \mathcal{J}} x_{i, \ell} \beta_{A, i, \ell, \ell^{\prime}} \beta_{A, i, \ell, \ell^{\prime}}^{*} y_{i, \ell^{\prime}}^{*}$ and $x_{\ell}:=\sum_{i} x_{i, \ell} u_{i} \otimes v_{i, \ell}, y_{\ell}:=$ $\sum_{i} y_{i, \ell} u_{i} \otimes v_{i, \ell}$. The resulting $h_{A}$ constructed in this way is manifestly also a standard hermitian structure.

The construction of the hermitian structure $h_{B}$ is similarly obtained, using $h_{B}^{\mathcal{E}_{B}}$, the hermitian metrics coming from the arithmetic vector bundle $\mathcal{E}_{B}$. One defines $h_{B}: \mathcal{E}_{\sigma} \rightarrow B_{\sigma}$ by setting

$$
h_{B}(x, y):=\sum_{j} x_{j} \beta_{B, j} \beta_{B, j}^{*} y_{j}^{*}=\sum_{\ell} h_{B}^{\mathcal{E}_{B}}\left(x_{\ell}, y_{\ell}\right),
$$

where $\beta_{B, j} \in B^{*}$ are the elements of the standard hermitian structure $h_{B}^{\mathcal{E}_{B}}, x=$ $\sum_{\ell \in \mathcal{I}} v_{j, \ell}^{\prime} \otimes u_{j}^{\prime} x_{j, \ell}$ with $\left\{v_{j, \ell}^{\prime}\right\}_{\ell \in \mathcal{I}}$ a basis of $\mathcal{E}_{A}$ as a $\mathbb{K}$-vector space and $x_{j}=\left(x_{j, \ell}\right)_{\ell}$ for fixed $j$ and $x_{\ell}=\sum_{j} v_{j, \ell}^{\prime} \otimes u_{j}^{\prime} x_{j, \ell}$ for fixed $\ell$. Due to the fact that $h_{A}^{\mathcal{E}_{A}}$ and $h_{B}^{\mathcal{E}_{B}}$ are standard hermitian metrics, $h_{A}$ and $h_{B}$ also satisfy the conditions in Definition 4.6 and Definition 4.4.

4.5. A height function on hermitian bimodules. We now discuss how to introduce a height function on the hermitian bimodules in ${ }_{\left(A, \mathcal{O}_{A}\right)} \mathcal{H}_{\left(B, \mathcal{O}_{B}\right)}$, which we regard as our action functional for weighting the bimodules, thought of as "maps" of a noncommutative sigma model with target a noncommutative space $X_{\left(A, \mathcal{O}_{A}\right)}^{n c}$ (which we can think of as another manifestation of the noncommutative arithmetic curve $\left.\operatorname{Spec}\left(\mathcal{O}_{A}\right)\right)$. We present here the general form the height function should take and we complete our definition of height after a further discussion of norms and volumes in $\$ 4.7$ and 4.8 .

First observe that, in the case of a "strong" hermitian bimodule, in the sense of Definition 4.7, one can proceed as in [6] to define a height function, as we recalled above, but taking into account the presence of two hermitian structures $h=\left(h_{A}, h_{B}\right)$.

For $\sigma$ an archimedean place of $\mathbb{K}$, let $\mathbb{K}_{\sigma}:=\mathbb{R}$ if $\sigma$ is a real embedding and $\mathbb{K}_{\sigma}:=\mathbb{C}$ if it is a complex embedding.

Let $\operatorname{vol}_{h_{A}, \sigma}$ denote a volume in $A_{\sigma}^{n}$ determined by the norm associated to the bilinear form $\operatorname{Tr}_{A_{\sigma} \mid \mathbb{K}_{\sigma}} \circ h_{A}$ (see $\$ 4.7$ below for a discussion of volumes associated to norms.) Let $\mathcal{V}$ be a free $A$-submodule of $A^{n}$ of rank $r_{A}$. We write the normalized and nonnormalized heights, respectively, as

$$
\bar{H}_{\mathcal{O}_{A}, h_{A}, \sigma}(\mathcal{V}):=\frac{\operatorname{vol}_{h_{A}, \sigma}\left(\mathcal{V} \cap \mathcal{O}_{A}^{n}\right)}{\operatorname{vol}_{h_{A}, \sigma}\left(\mathcal{O}_{A}\right)^{r_{A, \sigma}}} \quad \text { and } H_{\mathcal{O}_{A}, h_{A}, \sigma}(\mathcal{V}):=\operatorname{vol}_{h_{A}, \sigma}\left(\mathcal{V} \cap \mathcal{O}_{A}^{n}\right)
$$

The normalized height agrees with the height $H_{\mathcal{O}_{A}}$ of [6] when $h_{A}$ is the standard hermitian metric $h_{n}$ and $\sigma$ is a real embedding. 
Definition 4.10. Let $\left(\mathcal{E}, h_{A}, h_{B}\right)$ be a hermitian bimodule in ${ }_{\left(A, \mathcal{O}_{A}\right)} \mathcal{H}_{\left(B, \mathcal{O}_{B}\right)}$, as in Definition 4.6. We define the normalized and non-normalized heights of $\left(\mathcal{E}, h_{A}, h_{B}\right)$ as

$$
\bar{H}\left(\mathcal{E}, h_{A}, h_{B}\right):=\prod_{\sigma} \frac{\bar{H}_{\mathcal{O}_{B}, h_{B}, \sigma}\left(\mathcal{E}_{B}\right)}{\bar{H}_{\mathcal{O}_{A}, h_{A}, \sigma}\left(\mathcal{E}_{A}\right)} \quad \text { and } \quad H\left(\mathcal{E}, h_{A}, h_{B}\right):=\prod_{\sigma} \frac{H_{\mathcal{O}_{B}, h_{B}, \sigma}\left(\mathcal{E}_{B}\right)}{H_{\mathcal{O}_{A}, h_{A}, \sigma}\left(\mathcal{E}_{A}\right)}
$$

where $\sigma$ ranges over the archimedean places of $\mathbb{K}$, with $\mathcal{E}_{A}=A \otimes_{\mathcal{O}_{A}} \mathcal{E}$ and $\mathcal{E}_{B}=$ $\mathcal{E} \otimes_{\mathcal{O}_{B}} B$

In order to have a meaningful minimization problem for (4.4) one needs to minimize over classes of hermitian bimodules with a fixed positive lower bound on $\bar{H}_{\mathcal{O}_{A}, h_{A}, \sigma}\left(\mathcal{E}_{A}\right)$ while minimizing over $\bar{H}_{\mathcal{O}_{B}, h_{B}, \sigma}$. For modules of fixed rank, minimizing with respect to the normalized or non-normalized height is the same.

In our setting we also want to consider a notion of relative height in the case of finite projective modules instead of free modules, so that Definition 4.10 above can be applied to the more general class of hermitian bimodules of Definition 4.6 and not only to the strong ones.

4.6. The rank element. We will discuss more in detail how to obtain a suitable volume computation in 4.7 and 84.8 . We discuss here the rank $r_{A}$ in (4.3). Given a finite projective left module $\mathcal{P}$ over a unital noncommutative ring $R$, there is a notion of trace of an endomorphism, and of rank element, given by the trace of the identity, [19]. Consider the identification

$$
\vartheta: \mathcal{P}^{\vee} \otimes_{R} \mathcal{P} \stackrel{\simeq}{\rightarrow} \operatorname{Hom}_{R}(\mathcal{P}, \mathcal{P}),
$$

with $\mathcal{P}^{\vee}=\operatorname{Hom}_{R}(\mathcal{P}, R)$ and $\vartheta(\eta \otimes x)(y)=\eta(y) x$. Also consider the morphism

$$
\pi: \mathcal{P}^{\vee} \otimes_{R} \mathcal{P} \rightarrow H H_{0}(R)=R /[R, R]
$$

with $[R, R]$ the additive commutators subgroup of $R$ and $\pi(\eta \otimes x)=\eta(x) \bmod [R, R]$.

Definition 4.11. Given a finite projective left module $\mathcal{P}$ over a unital noncommutative ring $R$, the trace of an endomorphism $f \in \operatorname{Hom}_{R}(\mathcal{P}, \mathcal{P})$ is the element in $H H_{0}(R)$ obtained by setting $\operatorname{Tr}_{R}(f):=\pi\left(\vartheta^{-1}(f)\right)$.

In the case where $R=M_{n}(D)$ is a matrix algebra over a division algebra over a field $\mathbb{K}$, an element $X \in M_{n}(D)$ is in the commutator $\left[M_{n}(D), M_{n}(D)\right]$ iff its trace is in $[D, D]$. Moreover (Corollary 13.6 of [29]) $D$ is generated as a division algebra by $Z(D)$ and all the commutators in $[D, D]$. If $D$ is central, $Z(D)=\mathbb{K}$, then the trace elements can be seen as scalars in $\mathbb{K}$. More generally, the trace elements determine scalars in the field extension $Z(D)$ of $\mathbb{K}$. In particular, for $f=$ id the identity, $\operatorname{Tr}_{R}(\mathrm{id})=: r_{R}(\mathcal{P})$ is the rank element of $\mathcal{P}$. If $\mathcal{P}=R e$ with $e$ an idempotent in $R$, then $r_{R}(\mathcal{P})$ is the class of $e$ in $H H_{0}(R)$ and it is independent of the choice of idempotent: if $A e \simeq A e^{\prime} \simeq \mathcal{P}$ then $e \sim e^{\prime}$ in $H H_{0}(R)$, see [19] for this and more general cases. This rank element is known as the Hattori-Stallings rank and the more general traces as in Definition 4.11 are usually referred to as the Hattori-Stallings trace.

Thus, for our purposes, we summarize the above in the following statement. 
Lemma 4.12. Let $A$ be a semisimple finite dimensional algebra over a number field $\mathbb{K}$ and $\mathcal{E}$ a finite projective (left) $A$-module (see Remark 4.8). The rank element $r_{A}=r_{A}(\mathcal{E})$ is the class in $H H_{0}(A)=A /[A, A]$ given by the trace of the identity, $\operatorname{Tr}_{R}(\mathrm{id})=: r_{A}(\mathcal{E})$. A choice of an archimedean embedding $\sigma: \mathbb{K} \hookrightarrow \mathbb{C}$ (or $\mathbb{R}$ ) and a compatible embedding $\tilde{\sigma}$ of the extension $\mathbb{L}$ of $\mathbb{K}$ generated by the centers $Z\left(A_{i}\right)$ of the simple components $A_{i}$ of $A$ determine an associated rank $r_{A, \sigma}=\tilde{\sigma}\left(r_{A}\right) \in \mathbb{C}$ (or $\left.\mathbb{R}\right)$.

Proof. The center $Z(A)$ of a semisimple algebra is a finite direct product of field extensions of $\mathbb{K}, Z(A)=\oplus_{i} \mathbb{L}_{i}$, with $A=\oplus_{i} M_{n_{i}}\left(D_{i}\right)$ and $\mathbb{L}_{i}=Z\left(D_{i}\right)=H H_{0}\left(M_{n_{i}}\left(D_{i}\right)\right)$. The rank element $r_{A} \in H H_{0}(A)$ is then given by a collection of elements $r_{A, i} \in \mathbb{L}_{i}$. Given an embedding $\sigma: \mathbb{K} \hookrightarrow \mathbb{C}$ (or into $\mathbb{R}$ for a real place), let $\tilde{\sigma}: \mathbb{L} \hookrightarrow \mathbb{C}$ (or $\mathbb{R}$ ) be an extension of $\sigma$ to an embedding of the smallest extension $\mathbb{L}$ of $\mathbb{K}$ that contains all the $\mathbb{L}_{i}$. Then setting $\sigma\left(r_{A}\right):=\sum_{i} \tilde{\sigma}\left(r_{A, i}\right)$ assigns a complex (or real) number to the rank element $r_{A} \in H H_{0}(A)$.

4.7. Norms and volumes. There are different possible ways of associating to a finite dimensional normed space of dimension $N$ a volume form (and $k$-volume forms for $k \leq N)$ : some of these, such as Busemann volume, Holmes-Thompson volume, Gromov mass, etc. are summarized in [1]. We discuss briefly the case of the Gromov mass, to illustrate what conditions need to be satisfied for a good notion of volume.

Let $(V,\|\cdot\|)$ be a normed space of dimension $N$. The Gromov $N$-mass is defined as a function $\mu_{N}: \Lambda^{N} V \rightarrow \mathbb{R}_{+}$

$$
\mu_{N}(a):=\inf \left\{\prod_{i=1}^{N}\left\|v_{i}\right\| \mid v_{1} \wedge \cdots \wedge v_{N}=a\right\} .
$$

In order to define in a similar way $k$-volumes for $k<N=\operatorname{dim} V$, let $\Lambda_{s}^{k} V \subset \Lambda^{k} V$ be the subset consisting of the "simple" $k$-vectors, that is, those of the form $v_{1} \wedge \cdots \wedge v_{k}$, with $v_{i} \in V$. In order to define a $k$-volume, it suffices to assign a volume to elements of $\Lambda_{s}^{k} V$. Thus, one defines a $k$-volume as a homogeneous continuous function $\mu_{k}$ : $\Lambda_{s}^{k} V \rightarrow \mathbb{R}_{+}$with $\mu_{s}(\lambda a)=|\lambda| \mu_{s}(a)$ for $\lambda \in \mathbb{R}^{*}$. The analog of the Gromov mass $\mu_{N}$ is given by

$$
\mu_{k}(a):=\inf \left\{\prod_{i=1}^{k}\left\|v_{i}\right\| \mid v_{1} \wedge \cdots \wedge v_{k}=a\right\} .
$$

In this notion of $k$-volumes, the constrained optimization of the norms is designed to avoid redundancies that would make the norm unnecessarily large, such as adding to a vector in a basis a large multiple of another basis vector, and also to avoid changes to the norm due to an arbitrary scaling of a basis. The problem simplifies if the normed vector space $V$ is endowed with a more rigid structure, given by the choice of a lattice $V_{\mathbb{Z}} \subset V$. In this case, if we want to assign $k$-volumes to elements of $\Lambda_{s}^{k} V_{\mathbb{Z}}$, we can choose a different form of optimization of the norms by a priori 
selecting a "shortest basis" $\left\{v_{1}, \ldots, v_{N}\right\}$ for the lattice $V_{\mathbb{Z}}$. One can then define

$$
\mu_{k}\left(v_{i_{1}} \wedge \cdots \wedge v_{i_{k}}\right):=\prod_{\ell=1}^{k}\left\|v_{i_{\ell}}\right\| .
$$

for the basis elements $\left\{v_{i_{1}} \wedge \cdots \wedge v_{i_{k}}\right\}$ of the $\mathbb{Z}$-module $\Lambda^{k} V_{\mathbb{Z}}$. The shortest basis for a lattice is the basis with the smallest possible orthogonality defect

$$
\delta\left(\left\{v_{i}\right\}\right)=\frac{\prod_{i=1}^{N}\left\|v_{i}\right\|}{d\left(V_{\mathbb{Z}}\right)},
$$

where $d\left(V_{\mathbb{Z}}\right)$ is the lattice constant, which is equal to $\operatorname{det}(B)$, the determinant of the matrix $B=\left(v_{1}, \ldots, v_{N}\right)$ formed by the vectors $v_{i}$, in the case where $V_{\mathbb{Z}}$ is of rank $N$. For rank $k \leq N$, one can still define the defect in the same way with $d\left(V_{\mathbb{Z}}\right)=\sqrt{\operatorname{det}\left(B^{*} B\right)}$, which measures the Euclidean $k$-volume of the fundamental parallelepiped determined by the basis $\left\{v_{i}\right\}$. The defect is therefore the ratio between the volume defined as in $\mu_{k}\left(v_{i_{1}} \wedge \cdots \wedge v_{i_{k}}\right)$ and the Euclidean volume, and $\mu_{k}$ is computed on the choice of basis where this discrepancy is as small as possible. A lattice reduction algorithm that approximates the shortest basis for a lattice is developed in [30].

Note that the quantity $d\left(V_{\mathbb{Z}}\right)=\sqrt{\operatorname{det}\left(B^{*} B\right)}$ has itself an interpretation in terms of heights, since it is the archimedean part of the height of the matrix $B$ seen as a point in a Grassmannian, see Remark 2.8.7 of [5].

Definition 4.13. Let $\Lambda \subset V$ be a lattice of rank $k$ inside a $\mathbb{K}$-vector space of dimension $N \geq k$, namely a finitely generated torsion free $\mathcal{O}_{\mathbb{K}}$-module such that $\mathbb{K} \Lambda$ spans a $k$-dimensional subspace of $V$. Given a norm $\|\cdot\|$ on $V$, the $k$-volume of $\Lambda$ is

$$
\operatorname{vol}_{k}(\Lambda):=\mu_{k}\left(v_{1} \wedge \cdots \wedge v_{k}\right)=\prod_{\ell=1}^{k}\left\|v_{\ell}\right\|,
$$

where $\left\{v_{1}, \ldots, v_{k}\right\}$ is a shortest basis for $\Lambda$.

Lemma 4.14. Let $\left(A, \mathcal{O}_{A}\right)$ be a pair of a semisimple finite dimensional algebra $A$ over a number field $\mathbb{K}$ and an order $\mathcal{O}_{A}$. Let $\sigma$ be an archimedean place of $\mathbb{K}$ with $\mathbb{K}_{\sigma}$ equal to $\mathbb{R}$ or $\mathbb{C}$ for real and complex places, respectively and let $h_{A}: A_{\sigma} \times A_{\sigma} \rightarrow A_{\sigma}$ be a hermitian structure. Let $\mathcal{E}$ be a (left) $\mathcal{O}_{A}$-module. The notion of volume of lattices of Definition 4.13 and the rank element $r_{A}(\mathcal{E})$ of Lemma 4.12 determine the quantity $\operatorname{vol}_{h_{A}, \sigma}\left(\mathcal{O}_{A}\right)^{r} r_{A, \sigma}$ of (4.3) .

Proof. As shown in [6] all hermitian structures on $A_{\sigma}=A \otimes_{\mathbb{K}} \mathbb{K}_{\sigma}$ are standard in the sense of Definition 4.4, hence there is an element $\beta \in A^{*}$ such that $h_{A}(x, y)=$ $x^{*} \beta^{*} \beta y$, and $h_{A}(x, x)$ is a positive element for all $x \in A_{\sigma}$. Thus, we have $\|x\|^{2}:=$ $\operatorname{Tr}\left(h_{A}(x, x)\right) \geq 0$ for all $x \in A_{\sigma}$. By Definition 4.1, an order $\mathcal{O}_{A}$ in $A$ is a $\mathcal{O}_{\mathbb{K}}$-lattice with $\mathbb{K} \mathcal{O}_{A}=A$ as a $\mathbb{K}$-vector space, hence the norm determined by $h_{A}$ also determines a volume, as in (4.7), of the lattice $\mathcal{O}_{A}$ with $k=\operatorname{dim}_{\mathbb{K}} A$, the dimension as $\mathbb{K}$-vector 
space. With $r_{A, \sigma}=\tilde{\sigma}\left(r_{A}(\mathcal{E})\right)$ as in Lemma 4.12, we then obtain the expression in (4.3) that generalizes to our setting the height function (4.2) used in [6].

We will see in the following how to obtain also a good definition of the volumes $\operatorname{vol}_{h_{A}, \sigma}\left(\mathcal{E} \cap \mathcal{O}_{A}^{n}\right)$, suitable for extending the definition of the height function as in (4.3) to our notion of arithmetic vector bundles.

4.8. Volumes, traces and heights. We look here more explicitly at the height function defined in (4.4) by writing more explicitly the traces and volume forms on the bimodules. We will assume here that hermitian structures as standard as in Definition 4.4.

Proposition 4.15. Consider algebras of the form

$$
A=\oplus_{i} M_{n_{i}}\left(D_{i}\right) \quad \text { and } \quad B=\oplus_{j} M_{m_{j}}\left(D_{j}^{\prime}\right)
$$

where $D_{i}, D_{j}^{\prime}$ are division algebras over the number field $\mathbb{K}$. We also assume that an order $\mathcal{O}_{A}$ (respectively, $\mathcal{O}_{B}$ ) has been chosen. Let $\mathcal{E}$ be an $\mathcal{O}_{A}-\mathcal{O}_{B}$ bimodule. For every choice of a (standard) hermitian structure $\left(h_{A}, h_{B}\right)$ and archimedean place $\sigma$ of $\mathbb{K}$, there are associated volumes $\operatorname{vol}_{h_{A}, \sigma}\left(j_{A}(\mathcal{E}) \cap \mathcal{O}_{A}^{n_{A}}\right)$ and $\operatorname{vol}_{h_{B}, \sigma}\left(j_{B}(\mathcal{E}) \cap \mathcal{O}_{B}^{n_{B}}\right)$, where $j_{A}: \mathcal{E} \hookrightarrow A^{n_{A}}$ and $j_{B}: \mathcal{E} \hookrightarrow B^{n_{B}}$ realize the projective $A$-module (respectively, $B$-module) as a summand of a free module.

Proof. For semisimple algebras all left (respectively, right) modules are semisimple ([29], Theorem 2.5), hence we can consider $A-B$ bimodules that are of the form

$$
\mathcal{E}=\oplus_{i, j} D_{i}^{n_{i} N_{i}} \otimes D_{j}^{\prime m_{j} M_{j}}
$$

with the obvious left and right action of the respective matrix algebras.

Standard hermitian structures $h_{A}: \mathcal{E} \times \mathcal{E} \rightarrow A$ and $h_{B}: \mathcal{E} \times \mathcal{E} \rightarrow B$ are obtained as follows.

A choice of elements $\beta_{A, i} \in M_{n_{i}}\left(D_{i}\right)^{*}$ and $\beta_{B, j} \in M_{m_{j}}\left(D_{j}^{\prime}\right)^{*}$. determines hermitian forms $h_{A, i}: D_{i}^{n_{i}} \times D_{i}^{n_{i}} \rightarrow D_{i}$ and $h_{B, j}: D_{j}^{\prime m_{j}} \times D_{j}^{\prime m_{j}} \rightarrow D_{j}^{\prime}$ by

$$
h_{A, i}(x, y):=x \beta_{A, i} \beta_{A, i}^{*} y^{*} \quad \text { and } \quad h_{B, j}(u, v):=u^{*} \beta_{B, j}^{*} \beta_{B, j} v
$$

where $x=\left(x_{1}, \ldots, x_{n_{i}}\right)$ in $D_{i}^{n_{i}}$ (as a column vector) with $x^{*}$ the transpose (row vector) $\left(x_{1}^{*}, \ldots, x_{n_{i}}^{*}\right)$, and similarly for $h_{B, j}$. These extend similarly to hermitian forms $h_{A, i}: D_{i}^{n_{i} N_{i}} \times D_{i}^{n_{i} N_{i}} \rightarrow D_{i}$ and $h_{B, j}: D_{j}^{\prime m_{j} M_{j}} \times D_{j}^{\prime m_{j} M_{j}} \rightarrow D_{j}^{\prime}$.

Here we are using the notation $*$ both for an involution on $M_{n}(D)$ and for an involution on $D$, assuming compatible involutions on both. For example, if $M_{n}(D)$ is a central simple algebra, then it admits an involution that is the identity on the field $\mathbb{K}$ (involution of the first kind) iff the division algebra $D$ admits such an involution, with the involution on $M_{n}(D)$ given by the involution on $D$ applied to all entries, combined with matrix transposition.

These hermitian structures on the $D_{i}^{n_{i} N_{i}}$ and $D_{j}^{\prime m_{j} M_{j}}$ determine hermitian structures $h_{A}: \mathcal{E} \times \mathcal{E} \rightarrow A$ and $h_{B}: \mathcal{E} \times \mathcal{E} \rightarrow B$ as shown in Lemma 4.9, In turn, for 
any archimedean place $\sigma$ of $\mathbb{K}$, these hermitian forms determine hermitian structures $h_{A}: \mathcal{E}_{\sigma} \times \mathcal{E}_{\sigma} \rightarrow A_{\sigma}$, and similarly for $h_{B}$.

Let $\left\{e_{i, k}\right\}$ denote a basis of $D_{i}^{n_{i} N_{i}}$ as a left $M_{n_{i}}\left(D_{i}\right)$-module and similarly let $\left\{e_{j, k^{\prime}}^{\prime}\right\}$ denote a basis of $D_{j}^{\prime m_{j} M_{j}}$ as a right $M_{m_{j}}\left(D_{j}^{\prime}\right)$-module. Let $\left\{v_{j, \ell^{\prime}}^{\prime}\right\}$ be a basis of $D_{j}^{\prime m_{j} M_{j}}$ as a $\mathbb{K}$-vector space and $\left\{v_{i, \ell}\right\}$ be a basis of $D_{i}^{n_{i} N_{i}}$ as a $\mathbb{K}$-vector space, so that, as in Lemma 4.9. we can consider $u_{i, j ; k, \ell^{\prime}}=e_{i, k} \otimes v_{j, \ell^{\prime}}^{\prime}$ and $u_{i, j ; \ell, k^{\prime}}^{\prime}=v_{i, \ell} \otimes e_{j, k^{\prime}}^{\prime}$ as bases, respectively, of $\mathcal{E}$ as a left $A$-module and as a right $B$-module, so that we can write elements $\xi \in \mathcal{E}$ in the form $\xi=\sum_{i, j, k, \ell^{\prime}} a_{i, j ; k, \ell^{\prime}} u_{i, j ; k, \ell^{\prime}}$ with $a_{i, j ; k, \ell^{\prime}} \in M_{n_{i}}\left(D_{i}\right)$ or in the form $\xi=\sum_{i, j, \ell, k^{\prime}} u_{i, j ; \ell, k^{\prime}}^{\prime} b_{i, j ; \ell, k^{\prime}}$ with $b_{i, j ; \ell, k^{\prime}} \in M_{m_{j}}\left(D_{j}^{\prime}\right)$.

Since $\mathcal{E}$ is a finite projective module, both as a left $A$-module and as a right $B$-module (see Remark 4.8), we can use a choice of bases to determine embeddings $j_{A}: \mathcal{E} \hookrightarrow A^{n_{A}}$ and $j_{B}: \mathcal{E} \hookrightarrow B^{n_{B}}$, which realizes $\mathcal{E}$ as $\mathcal{E} \simeq \mathcal{O}_{A}^{n_{A}} e_{A}$ with an idemponent $e_{A} \in M_{n_{A}}(A)$ with components $\left(e_{A}\right)_{i j}$ determined by the basis elements, and similarly for the $B$-module structure.

If the bimodule $\mathcal{E}$ is arithmetic, namely it is obtained as an $A$ - $B$ bimodule by change of coefficients $\mathcal{E}=A \otimes_{\mathcal{O}_{A}} \mathcal{E}_{\mathcal{O}} \otimes_{\mathcal{O}_{B}} B$ from an $\mathcal{O}_{A^{-}} \mathcal{O}_{B}$ bimodule $\mathcal{E}_{\mathcal{O}}$, then it is possible to choose the bases in such a way that they are in $\mathcal{E}_{\mathcal{O}}$, with the $\left\{e_{i, k}\right\}$ and $\left\{e_{j, k^{\prime}}^{\prime}\right\}$ giving $\mathcal{O}_{A}$ and $\mathcal{O}_{B}$ bases, respectively, and the $\left\{v_{i, \ell}\right\}$ and $\left\{v_{j, \ell^{\prime}}^{\prime}\right\}$ bases of $\mathcal{O}_{\mathbb{K}^{-}}$-lattices, so that the resulting $\left\{u_{i, j ; k, \ell^{\prime}}\right\}$ and $\left\{u_{i, j ; k, \ell^{\prime}}\right\}$ give bases of $\mathcal{E}_{\mathcal{O}}$ as a left and right $\mathcal{O}_{A}$ and $\mathcal{O}_{B}$ module, respectively. Let $\mathcal{B}, \mathcal{B}^{\prime}$ denote the set of all such bases and let $u_{\min }, u_{\min }^{\prime}$ be shortest basis

$$
\delta\left(u_{\min }\right)=\inf _{u \in \mathcal{B}} \delta(u) \quad \text { and } \quad \delta\left(u_{\min }^{\prime}\right)=\inf _{u^{\prime} \in \mathcal{B}^{\prime}} \delta\left(u^{\prime}\right)
$$

with $\delta$ as in (4.6), where the norm $\|\cdot\|=\operatorname{Tr}\left(h_{A}(\cdot, \cdot)\right)^{1 / 2}$ (or $h_{B}$, respectively) and the lattice constant $d$ is computed as the Euclidean volume as $\mathbb{Z}$-lattices.

We can then consider $j_{A}\left(\mathcal{E}_{\mathcal{O}}\right) \cap \mathcal{O}_{A}^{n_{A}}$ and $j_{B}\left(\mathcal{E}_{\mathcal{O}}\right) \cap \mathcal{O}_{B}^{n_{B}}$. These can be viewed as $\mathcal{O}_{A}$-module (respectively, $\mathcal{O}_{B}$-module) and as $\mathcal{O}_{\mathbb{K}}$-modules. Let $k_{A}$, $k_{B}$ denote, respectively, their ranks as $\mathcal{O}_{\mathbb{K}}$-modules.

We can then assign a volume to $j_{A}\left(\mathcal{E}_{\mathcal{O}}\right) \cap \mathcal{O}_{A}^{n_{A}}$ and $j_{B}\left(\mathcal{E}_{\mathcal{O}}\right) \cap \mathcal{O}_{B}^{n_{B}}$ as in (4.7), computed with respect to a shortest basis, in the sense discussed above.

Thus, we obtain a well defined height functions

$$
\bar{H}_{\mathcal{O}_{A}, h_{A}, \sigma}(\mathcal{E})=\frac{\operatorname{vol}_{h_{A}, \sigma}\left(j_{A}(\mathcal{E}) \cap \mathcal{O}_{A}^{n_{A}}\right)}{\operatorname{vol}_{h_{A}, \sigma}\left(\mathcal{O}_{A}\right)^{\sigma\left(r_{A}\right)}} \quad \text { and } \quad \bar{H}_{\mathcal{O}_{B}, h_{B}, \sigma}(\mathcal{E})=\frac{\operatorname{vol}_{h_{B}, \sigma}\left(j_{B}(\mathcal{E}) \cap \mathcal{O}_{B}^{n_{B}}\right)}{\operatorname{vol}_{h_{B}, \sigma}\left(\mathcal{O}_{B}\right)^{\sigma\left(r_{B}\right)}}
$$

that can be used in the height function of Definition 4.10 .

4.9. Multiplicative property. The issue here is that, while the height function (4.9) assigns to bimodules an "energy" as we want, it only sees bimodules as forming a set, while we also want to keep track of the structure of our functor of points as a category. This means that we want a height function that is in a natural way compatible with the composition operation given by the tensor product of bimodules, 
while still being based on an appropriate notion of volumes. To obtain a simple setting that satisfies this property, we will restrict the class of bimodules and the choice of basis used to compute the height.

Arithmetic hermitian bimodules behave in the following way under tensor product.

Lemma 4.16. The tensor product $\left(\mathcal{E}, h_{A}, h_{C}\right)$ in ${ }_{\left(A, \mathcal{O}_{A}\right)} \mathcal{H}_{\left(C, \mathcal{O}_{C}\right)}$ of arithmetic hermitian bimodules $\left(\mathcal{E}^{\prime}, h_{A}^{\prime}, h_{B}^{\prime}\right)$ in ${ }_{\left(A, \mathcal{O}_{A}\right)} \mathcal{H}_{\left(B, \mathcal{O}_{B}\right)}$ and $\left(\mathcal{E}^{\prime \prime}, h_{B}^{\prime \prime}, h_{C}^{\prime \prime}\right)$ in ${ }_{\left(B, \mathcal{O}_{B}\right)} \mathcal{H}_{\left(C, \mathcal{O}_{C}\right)}$ is given by $\mathcal{E}=\mathcal{E}^{\prime} \otimes_{\mathcal{O}_{B}} \mathcal{E}^{\prime \prime}$ with the hermitian structures

$$
\begin{aligned}
& h_{A}\left(x^{\prime} \otimes x^{\prime \prime}, y^{\prime} \otimes y^{\prime \prime}\right):=h_{A}^{\prime}\left(x^{\prime} h_{B}^{\prime \prime}\left(x^{\prime \prime}, y^{\prime \prime}\right), y^{\prime}\right) \\
& h_{C}\left(x^{\prime} \otimes x^{\prime \prime}, y^{\prime} \otimes y^{\prime \prime}\right):=h_{C}^{\prime \prime}\left(x^{\prime \prime}, h_{B}^{\prime}\left(x^{\prime}, y^{\prime}\right) y^{\prime \prime}\right) .
\end{aligned}
$$

Proof. To prove this Lemma, one needs to show that $\left(\mathcal{E}, h_{A}, h_{C}\right)$ satisfies all the properties of Definition 4.6. By construction, $\mathcal{E}=\mathcal{E}^{\prime} \otimes_{\mathcal{O}_{B}} \mathcal{E}^{\prime \prime}$ is an $\mathcal{O}_{A}-\mathcal{O}_{C}$ bimodule since $\mathcal{E}^{\prime}$ is an $\mathcal{O}_{A}$-left module and $\mathcal{E}^{\prime \prime}$ is an $\mathcal{O}_{C}$-right module, and those two actions are compatible. In addition, Remark 4.8 implies that $A \otimes_{\mathcal{O}_{A}} \mathcal{E}$ and $\mathcal{E} \otimes_{\mathcal{O}_{C}} C$ are finite projective as $A$-module and $C$-module, respectively.

For any archimedean place $\sigma$ of $\mathbb{K}$, one needs to show that the bimodule $\mathcal{E}_{\sigma}:=$ $A_{\sigma} \otimes_{\mathcal{O}_{A}} \mathcal{E} \otimes_{\mathcal{O}_{C}} C_{\sigma}$ endowed with the pair of hermitian structures $h=\left(h_{A}, h_{B}\right)$, as defined above, with $h_{A}: \mathcal{E}_{\sigma} \times \mathcal{E}_{\sigma} \rightarrow A_{\sigma}$ and $h_{C}: \mathcal{E}_{\sigma} \times \mathcal{E}_{\sigma} \rightarrow C_{\sigma}$ satisfying all the conditions in Definition 4.6.

- For all $a \in A_{\sigma}$ and $\left(x^{\prime} \otimes x^{\prime \prime}, y^{\prime} \otimes y^{\prime \prime}\right) \in \mathcal{E}_{\sigma}$, the identities

$$
\begin{aligned}
h_{A}\left(a\left(x^{\prime} \otimes x^{\prime \prime}\right), y^{\prime} \otimes y^{\prime \prime}\right) & =h_{A}^{\prime}\left(a x^{\prime} h_{B}^{\prime \prime}\left(x^{\prime \prime}, y^{\prime \prime}\right), y^{\prime}\right) \\
& =a h_{A}^{\prime}\left(x^{\prime} h_{B}^{\prime \prime}\left(x^{\prime \prime}, y^{\prime \prime}\right), y^{\prime}\right) \\
& =a h_{A}\left(x^{\prime} \otimes x^{\prime \prime}, y^{\prime} \otimes y^{\prime \prime}\right)
\end{aligned}
$$

and

$$
\begin{aligned}
h_{A}\left(x^{\prime} \otimes x^{\prime \prime}, a\left(y^{\prime} \otimes y^{\prime \prime}\right)\right) & =h_{A}^{\prime}\left(x^{\prime} h_{B}^{\prime \prime}\left(x^{\prime \prime}, y^{\prime \prime}\right), a y^{\prime}\right) \\
& =h_{A}^{\prime}\left(x^{\prime} h_{B}^{\prime \prime}\left(x^{\prime \prime}, y^{\prime \prime}\right), y^{\prime}\right) a^{*} \\
& =h_{A}\left(x^{\prime} \otimes x^{\prime \prime}, y^{\prime} \otimes y^{\prime \prime}\right) a^{*}
\end{aligned}
$$

where the second last equalities hold since $h_{A}^{\prime}$ satisfies conditions in Definition 4.6.

- For all $x^{\prime} \otimes x^{\prime \prime} \in \mathcal{E}_{\sigma}$, the element $h_{A}\left(x^{\prime} \otimes x^{\prime \prime}, x^{\prime} \otimes x^{\prime \prime}\right)$ is a positive element in $A_{\sigma}$ since there is a $\beta_{B} \in B_{\sigma}^{*}$ such that

$$
\begin{aligned}
h_{A}\left(x^{\prime} \otimes x^{\prime \prime}, x^{\prime} \otimes x^{\prime \prime}\right) & =h_{A}^{\prime}\left(x^{\prime} h_{B}^{\prime \prime}\left(x^{\prime \prime}, x^{\prime \prime}\right), x^{\prime}\right) \\
& =h_{A}^{\prime}\left(x^{\prime} x^{\prime \prime} \beta_{B} \beta_{B}^{*} x^{\prime \prime *}, x^{\prime}\right) \\
& =h_{A}^{\prime}\left(x^{\prime} x^{\prime \prime} \beta_{B}, x^{\prime} x^{\prime \prime} \beta_{B}\right)
\end{aligned}
$$

and $h_{A}^{\prime}\left(x^{\prime} x^{\prime \prime} \beta_{B}, x^{\prime} x^{\prime \prime} \beta_{B}\right)$ is positive element in $A_{\sigma}$ given that $h_{A}^{\prime}$ satisfies condition in Definition 4.6.

And similarly reasoning holds for $h_{C}$, finishing the proof. 
Let $A$ be a semisimple finite dimensional algebra over a number field $\mathbb{K}$. Then by Wedderburn theorem $A=\oplus_{i} M_{n_{i}}\left(D_{i}\right)$ with division algebras $D_{i}$ over $\mathbb{K}$. The center $Z(A)$ is then a direct sum of number fields $\mathbb{L}_{i}$ that contain $\mathbb{K}$.

Definition 4.17. Let $\mathcal{A}_{\mathbb{K}}(Z)$ denote the set of all semisimple finite dimensional algebras $A$ over $\mathbb{K}$ such that $Z(A) \simeq Z$ with $Z$ a fixed $Z=\oplus_{i} \mathbb{L}_{i}$. Let $\mathcal{H}(\mathbb{K}, Z)$ denote the set of $A-B$ bimodules $\mathcal{E}$ satisfying the following properties

- The algebras $A, B$ are in $\mathcal{A}_{\mathbb{K}}(Z)$;

- $\mathcal{E}$ is an arithmetic $\left(A, \mathcal{O}_{A}\right)-\left(B, \mathcal{O}_{B}\right)$ hermitian bimodule, for some order $\mathcal{O}_{A}$ in $A$ and $\mathcal{O}_{B}$ in $B$, with hermitian structures $\left(h_{A}, h_{B}\right)$;

- There are bases $\left\{x_{i}\right\}$ and $\left\{y_{j}\right\}$ of $\mathcal{E}$ as a left $A$-module and a right $B$-module respectively, with the property that $h_{A}\left(x_{i}, x_{i}\right) \in Z$ and $h_{B}\left(y_{j}, y_{j}\right) \in Z$;

- As a $Z-Z$ bimodule $\mathcal{E}$ is a symmetric bimodule, namely $\lambda \xi=\xi \lambda$ for all $\xi \in \mathcal{E}$ and all $\lambda \in Z$.

Let $\mathcal{B}_{A}(\mathcal{E})$ and $\mathcal{B}_{B}(\mathcal{E})$ be, respectively, the set of all bases $\left\{x_{i}\right\}$ and bases $\left\{y_{j}\right\}$ that satisfy the condition above that $h_{A}\left(x_{i}, x_{i}\right) \in Z$ and $h_{B}\left(y_{j}, y_{j}\right) \in Z$ and such that $x_{i}, y_{j} \in \mathcal{E}_{\mathcal{O}}$, for all $i, j$.

Note that the sum $\sum_{i} h_{A}\left(x_{i}, x_{i}\right)$ is the trace of the identity endomorphism and is in $Z$ (identified with $H H_{0}(A)$ ) for an arbitrary choice of basis, and similarly for $\sum_{j} h_{B}\left(y_{j}, y_{j}\right)$. The condition that $h_{A}\left(x_{i}, x_{i}\right) \in Z$ and $h_{B}\left(y_{j}, y_{j}\right) \in Z$ is verified, for instance when $\mathcal{E}=A^{n_{A}} p$, for a projector $p \in M_{n_{A}}(Z(A))$ with diagonal and similarly $\mathcal{E}=p^{\prime} B^{n_{B}}$ with $p^{\prime} \in M_{n_{B}}(Z(B))$.

We can then considered a 2-category $\mathcal{H}(\mathbb{K}, Z)$ as follows.

Lemma 4.18. Let $\mathbb{K}$ be a number field and let $Z=\oplus_{i} \mathbb{L}_{i}$ be a given sum of number field extensions $\mathbb{L}_{i}$ of $\mathbb{K}$. The following data form a 2-category $\mathcal{H}(\mathbb{K}, Z)$ :

- Objects: pairs $\left(A, \mathcal{O}_{A}\right)$ of a semisimple finite dimensional algebra $A$ in $\mathcal{A}_{\mathbb{K}}(Z)$ with a $\mathcal{O}_{\mathbb{K}}$-order $\mathcal{O}_{A}$ in $A$;

- 1-morphisms $\operatorname{Mor}\left(\left(A, \mathcal{O}_{A}\right),\left(B, \mathcal{O}_{B}\right)\right)$ : arithmetic $\left(A, \mathcal{O}_{A}\right)-\left(B, \mathcal{O}_{B}\right)$ hermitian bimodules $\left(\mathcal{E}, h_{A}, h_{B}\right)$ with $\mathcal{E}$ in $\mathcal{H}(\mathbb{K}, Z)$;

- compositions of 1-morphisms $\left(\mathcal{E}, h_{A}^{\prime}, h_{B}^{\prime}\right)$ and $\left(\mathcal{F}, h_{B}^{\prime \prime}, h_{C}^{\prime \prime}\right)$ is

$$
\left(\mathcal{E}^{\prime} \otimes_{B} \mathcal{E}^{\prime \prime}, h_{A}, h_{C}\right),
$$

with $h_{A}, h_{C}$ as in Lemma 4.16.

- 2-morphisms: morphisms $\phi:\left(\mathcal{E}, h_{A}, h_{B}\right) \rightarrow\left(\tilde{\mathcal{E}}, \tilde{h}_{A}, \tilde{h}_{B}\right)$ of hermitian bimodules, namely morphisms of bimodules that preserve the hermitian forms, $\tilde{h}_{A}(\phi(x), \phi(y))=h_{A}(x, y)$ and $\tilde{h}_{B}(\phi(x), \phi(y))=h_{B}(x, y)$, with the property that of $\left\{x_{i}\right\}$ and $\left\{y_{j}\right\}$ are bases of $\mathcal{E} h_{A}\left(x_{i}, x_{i}\right) \in Z$ and $h_{B}\left(y_{j}, y_{j}\right) \in Z$, then $\left\{\phi\left(x_{i}\right)\right\}$ and $\left\{\phi\left(y_{j}\right)\right\}$ can be completed to bases of $\mathcal{F}$ with the same property;

- horizontal composition: tensor product of morphisms of hermitian bimodules

- vertical composition: composition of morphisms of bimodules. 
Proof. If $\left\{x_{i}\right\}$ and $\left\{y_{j}\right\}$ are bases of $\mathcal{E}$ as left $A$-module and a right $B$-module, satisfying $h_{A}^{\prime}\left(x_{i}, x_{i}\right) \in Z$ and $h_{B}^{\prime}\left(y_{j}, y_{j}\right) \in Z$, and $\left\{u_{\ell}\right\}$ and $\left\{v_{k}\right\}$ are bases of $\mathcal{F}$ as a left $B$-module and a right $C$-module, also satisfying $h_{B}^{\prime \prime}\left(u_{\ell}, u_{\ell}\right) \in Z$ and $h_{C}^{\prime \prime}\left(v_{k}, v_{k}\right) \in Z$, then $\left\{x_{i} \otimes u_{\ell}\right\}$ and $\left\{y_{j} \otimes v_{k}\right\}$ are bases for $\mathcal{E} \otimes_{B} \mathcal{F}$ as a left $A$-module and a right $C$-module satisfying

$$
h_{A}\left(x_{i} \otimes u_{\ell}, x_{i} \otimes u_{\ell}\right)=h_{A}^{\prime}\left(x_{i} h_{B}^{\prime \prime}\left(u_{i}, u_{i}\right), x_{i}\right)=h_{B}^{\prime \prime}\left(u_{i}, u_{i}\right) h_{A}^{\prime}\left(x_{i}, x_{i}\right) \in Z,
$$

where the second equality uses the symmetric property of $\mathcal{E}$ as a $Z$ - $Z$ bimodule, and similarly

$$
h_{C}\left(y_{j} \otimes v_{k}, y_{j} \otimes v_{k}\right)=h_{C}^{\prime \prime}\left(v_{k}, h_{B}^{\prime}\left(y_{j}, y_{j}\right) v_{k}\right)=h_{C}^{\prime \prime}\left(v_{k}, v_{k}\right) h_{B}^{\prime}\left(y_{j}, y_{j}\right) \in Z,
$$

similarly using the symmetry of $\mathcal{F}$ as $Z$ - $Z$ bimodule. The product $\mathcal{E} \otimes_{B} \mathcal{F}$ is also symmetric as $Z-Z$ bimodule. Thus, composition of 1-morphisms is well defined in $\mathcal{H}(\mathbb{K}, Z)$. The properties of the vertical and horizontal compositions of 2-morphisms are similarly verified.

Remark 4.19. Our (pointed) noncommutative functor of points $X_{\left(A, \mathcal{O}_{A}\right), \star}^{n c}$ can then be thought of as assigning to $\left(A, \mathcal{O}_{A}\right)$ with $A \in \mathcal{A}_{\mathbb{K}}(Z)$ the collection of all the arrows (1-morphisms) in $\mathcal{H}(\mathbb{K}, Z)$ with source $\left(A, \mathcal{O}_{A}\right)$.

We now define a height function as follows.

Definition 4.20. Suppose given an $\left(A, \mathcal{O}_{A}\right)-\left(B, \mathcal{O}_{B}\right)$ arithmetic hermitian bimodule $\left(\mathcal{E}, h_{A}, h_{B}\right)$ in $\mathcal{H}(\mathbb{K}, Z)$, with $A, B \in \mathcal{A}_{\mathbb{K}}(Z)$, and an archimedean place $\sigma: \mathbb{K} \rightarrow \mathbb{K}_{\sigma}$. The heights of $\left(\mathcal{E}, h_{A}, h_{B}\right)$ are given by

$$
\begin{aligned}
& H_{h_{A}, \sigma}(\mathcal{E}):=\inf _{\left\{x_{i}\right\} \in \mathcal{B}_{A}(\mathcal{E})} \prod_{i} \tilde{\sigma}\left(h_{A}\left(x_{i}, x_{i}\right)\right) \\
& H_{h_{B}, \sigma}(\mathcal{E}):=\inf _{\left\{y_{j}\right\} \in \mathcal{B}_{B}(\mathcal{E})} \prod_{j} \tilde{\sigma}\left(h_{B}\left(y_{j}, y_{j}\right)\right),
\end{aligned}
$$

with $\tilde{\sigma}$ an embedding of $Z$ in $\mathbb{C}$ that restricts to the embedding $\sigma: \mathbb{K} \hookrightarrow \mathbb{C}$. Correspondingly, we define a height function as in Definition 4.10 with

$$
H\left(\mathcal{E}, h_{A}, h_{B}\right):=\prod_{\sigma} \frac{H_{h_{B}, \sigma}(\mathcal{E})}{H_{h_{A}, \sigma}(\mathcal{E})} .
$$

Lemma 4.21. The height function defined as in (4.13) satisfies

$$
H\left(\mathcal{E} \otimes_{B} \mathcal{F}, h_{A}, h_{B}\right)=H\left(\mathcal{E}, h_{A}^{\prime}, h_{B}^{\prime}\right) \cdot H\left(\mathcal{F}, h_{B}^{\prime \prime}, h_{C}^{\prime \prime}\right),
$$

for $\left(\mathcal{E}, h_{A}^{\prime}, h_{B}^{\prime}\right)$ an $\left(A, \mathcal{O}_{A}\right)-\left(B, \mathcal{O}_{B}\right)$ hermitian bimodule and $\left(\mathcal{F}, h_{B}^{\prime \prime}, h_{C}^{\prime \prime}\right)$ an $\left(B, \mathcal{O}_{B}\right)$ $\left(C, \mathcal{O}_{C}\right)$ hermitian bimodule, both in $\mathcal{H}(\mathbb{K}, Z)$, and with $h_{A}, h_{B}$ as in Lemma 4.16 .

Proof. For any bases $\left\{x_{i}\right\}$ and $\left\{y_{j}\right\}$ of $\mathcal{E}$ in $\mathcal{B}_{A}(\mathcal{E})$ and $\mathcal{B}_{B}(\mathcal{E})$, respectively, and bases $\left\{u_{\ell}\right\}$ and $\left\{v_{k}\right\}$ of $\mathcal{F}$ in $\mathcal{B}_{B}(\mathcal{F})$ and $\mathcal{B}_{C}(\mathcal{F})$, by (4.10) and (4.11), we obtain a basis $\left\{x_{i} \otimes u_{\ell}\right\}$ in $\mathcal{B}_{A}\left(\mathcal{E} \otimes_{B} \mathcal{F}\right)$ and a basis $\left\{y_{j} \otimes v_{k}\right\}$ in $\mathcal{B}_{C}\left(\mathcal{E} \otimes_{B} \mathcal{F}\right)$. Moreover, also by (4.10) and (4.11)

$$
\prod_{i, \ell} \tilde{\sigma}\left(h_{A}\left(x_{i} \otimes u_{\ell}, x_{i} \otimes u_{\ell}\right)\right)^{1 / 2}=\prod_{i} \tilde{\sigma}\left(h_{A}^{\prime}\left(x_{i}, x_{i}\right)\right) \cdot \prod_{\ell} \tilde{\sigma}\left(h_{B}^{\prime \prime}\left(u_{\ell}, u_{\ell}\right)\right)
$$




$$
\prod_{j, k} \tilde{\sigma}\left(h_{C}\left(y_{j} \otimes v_{k}, y_{j} \otimes v_{k}\right)\right)=\prod_{j} \tilde{\sigma}\left(h_{B}^{\prime}\left(y_{j}, y_{j}\right)\right) \prod_{k} \tilde{\sigma}\left(h_{C}^{\prime \prime}\left(v_{k}, v_{k}\right)\right)
$$

hence minimizing over the choice of basis,

$$
\begin{aligned}
& H_{h_{A}, \sigma}\left(\mathcal{E} \otimes_{B} \mathcal{F}\right)=H_{h_{A}^{\prime}, \sigma}(\mathcal{E}) H_{h_{B}^{\prime \prime}, \sigma}(\mathcal{F}) \\
& H_{h_{C}, \sigma}\left(\mathcal{E} \otimes_{B} \mathcal{F}\right)=H_{h_{B}^{\prime}, \sigma}(\mathcal{E}) H_{h_{C}^{\prime \prime}, \sigma}(\mathcal{F}),
\end{aligned}
$$

so that

$$
H\left(\mathcal{E} \otimes_{B} \mathcal{F}, h_{A}, h_{C}\right)=\frac{H_{h_{C}, \sigma}\left(\mathcal{E} \otimes_{B} \mathcal{F}\right)}{H_{h_{A}, \sigma}\left(\mathcal{E} \otimes_{B} \mathcal{F}\right)}=\frac{H_{h_{B}^{\prime}, \sigma}(\mathcal{E})}{H_{h_{A}^{\prime}, \sigma}(\mathcal{E})} \cdot \frac{H_{h_{C}^{\prime \prime}, \sigma}(\mathcal{F})}{H_{h_{B}^{\prime \prime}, \sigma}(\mathcal{F})}
$$

4.10. Dynamics generated by the height function. We explain more clearly in this subsection in what sense the height function introduced in the previous subsection makes our functor of points $\left(B, \mathcal{O}_{B}\right) \mapsto X_{\left(A, \mathcal{O}_{A}\right)}^{n c}\left(B, \mathcal{O}_{B}\right)$ dynamical. To this purpose we take the point of view of quantum statistical mechanics and we define an algebra of observables over $X_{\left(A, \mathcal{O}_{A}\right)}^{n c}\left(B, \mathcal{O}_{B}\right)$ with a time evolution generated by the height function. The main idea is very similar to the algebra and dynamics constructed in [11], associated to the space of $\mathbb{Q}$-lattices with the commensurability relation, but the construction we present here is based on the convolution algebras of semigroupoids (small categories) and of 2-categories introduced in [43].

As we discussed in the introduction to this paper, we consider the height as a kind of "energy functional" on the "points" (here represented by bimodules) of a noncommutative space. We mentioned that this action functional makes our functor of points "dynamical". To make this idea more precise, we associate to the 2-category $\mathcal{H}(\mathbb{K}, Z)$ of bimodules whose 1-morphisms describe our functor of points, an algebra of observables and a time evolution determined by the action functional given by the height function (4.13).

As shown in [43] there are two convolution algebras $\mathcal{A}_{\mathcal{H}(\mathbb{K})}^{(1)}$ and $\mathcal{A}_{\mathcal{H}(\mathbb{K})}^{(2)}$ associated to a 2-category $\mathcal{H}(\mathbb{K})$. The first is based on 1-morphisms and their composition, and the second is based on 2-morphisms and their horizontal and vertical compositions.

The algebra $\mathcal{A}_{\mathcal{H}(\mathbb{K}, Z)}^{(1)}$ is given by finitely supported functions on the set of 1 morphisms of $\mathcal{H}(\mathbb{K}, Z)$, with the convolution product

$$
\left(f_{1} \star f_{2}\right)(\Phi)=\sum_{\Phi=\Phi^{\prime \prime} \star \Phi^{\prime}} f_{1}\left(\Phi^{\prime}\right) f_{2}\left(\Phi^{\prime \prime}\right),
$$

for $\Phi=\left(\mathcal{E}, h_{A}, h_{B}\right)$ in ${ }_{\left(A, \mathcal{O}_{A}\right)} \mathcal{H}_{\left(B, \mathcal{O}_{B}\right)}$ equal to the tensor product of $\Phi^{\prime}=\left(\mathcal{E}^{\prime}, h_{A}, h_{B}\right)$ in ${ }_{\left(A, \mathcal{O}_{A}\right)} \mathcal{H}_{\left(B, \mathcal{O}_{B}\right)}$ and $\Phi^{\prime \prime}=\left(\mathcal{E}^{\prime \prime}, h_{b}, h_{C}\right)$ in ${ }_{\left(B, \mathcal{O}_{B}\right)} \mathcal{H}_{\left(C, \mathcal{O}_{C}\right)}$, as in Lemma 4.16, with the composition $\Phi^{\prime \prime} \star \Phi^{\prime}$ of 1-morphisms given by the tensor product of hermitian bimodules. This algebra is associative but noncommutative. 
The algebra $\mathcal{A}_{\mathcal{H}(\mathbb{K}, Z)}^{(2)}$ is similarly defined but based on the two composition laws for 2-morphisms. It is given by finitely supported functions on the set of 2-morphisms (morphisms of hermitian bimodules) with two product operations

$$
\begin{aligned}
& \left(f_{1} \circ f_{2}\right)(\phi)=\sum_{\phi=\phi^{\prime \prime} \circ \phi^{\prime}} f_{1}\left(\phi^{\prime}\right) f_{2}\left(\phi^{\prime \prime}\right) \\
& \left(f_{1} \bullet f_{2}\right)(\phi)=\sum_{\phi=\phi^{\prime \prime} \bullet \phi^{\prime}} f_{1}\left(\phi^{\prime}\right) f_{2}\left(\phi^{\prime \prime}\right)
\end{aligned}
$$

where $\circ$ is the vertical composition of 2-morphisms

$$
\phi^{\prime \prime} \circ \phi^{\prime}:\left(\mathcal{E}, h_{A}, h_{B}\right) \stackrel{\phi^{\prime}}{\rightarrow}\left(\mathcal{E}^{\prime}, h_{A}^{\prime}, h_{B}^{\prime}\right) \stackrel{\phi^{\prime \prime}}{\rightarrow}\left(\mathcal{E}^{\prime \prime}, h_{A}^{\prime \prime}, h_{B}^{\prime \prime}\right)
$$

while $\phi^{\prime \prime} \bullet \phi^{\prime}$ is the horizontal composition deternined by the tensor product of hermitian bimodules.

Lemma 4.22. The height function (4.13) for hermitian bimodules in $\mathcal{H}(\mathbb{K}, Z)$ determines a time evolution on the convolution algebra $\mathcal{A}_{\mathcal{H}(\mathbb{K}, Z)}^{(1)}$ of 1 -morphisms of $\mathcal{H}(\mathbb{K}, Z)$.

Proof. We construct a 1-parameter family of automorphisms of $\mathcal{A}_{\mathcal{H}(\mathbb{K}, Z)}^{(1)}$, that is, a group homomorphism $\alpha: \mathbb{R} \rightarrow \operatorname{Aut}\left(\mathcal{A}_{\mathcal{H}(\mathbb{K}, Z)}^{(1)}\right)$ by taking

$$
\alpha_{t}(f)\left(\mathcal{E}, h_{A}, h_{B}\right)=H\left(\mathcal{E}, h_{A}, h_{B}\right)^{i t} f\left(\mathcal{E}, h_{A}, h_{B}\right)
$$

Clearly, $\alpha_{t+s}=\alpha_{t} \circ \alpha_{s}$. We need to check that the $\alpha_{t}$ are algebra homomorphisms

$$
\alpha_{t}\left(f_{1} \star f_{2}\right)\left(\mathcal{E}, h_{A}, h_{B}\right)=\left(\alpha_{t}\left(f_{1}\right) \star \alpha_{t}\left(f_{2}\right)\right)\left(\mathcal{E}, h_{A}, h_{B}\right) .
$$

The left-hand-side is given by $H\left(\mathcal{E}, h_{A}, h_{B}\right)^{i t} \sum f_{1}\left(\mathcal{E}^{\prime}, h_{A}^{\prime}, h_{C}^{\prime}\right) f_{2}\left(\mathcal{E}^{\prime \prime}, h_{C}^{\prime \prime}, h_{B}^{\prime \prime}\right)$, with the sum over all the decompositions of $\left(\mathcal{E}, h_{A}, h_{B}\right)$ as tensor product of $\left(\mathcal{E}^{\prime}, h_{A}^{\prime}, h_{C}^{\prime}\right)$ and $\left(\mathcal{E}^{\prime \prime}, h_{C}^{\prime \prime}, h_{B}^{\prime \prime}\right)$. The right hand side is given by

$$
\sum H\left(\mathcal{E}^{\prime}, h_{A}^{\prime}, h_{B}^{\prime}\right)^{i t} f_{1}\left(\mathcal{E}^{\prime}, h_{A}^{\prime}, h_{C}^{\prime}\right) H\left(\mathcal{E}^{\prime \prime}, h_{A}^{\prime \prime}, h_{B}^{\prime \prime}\right)^{i t} f_{2}\left(\mathcal{E}^{\prime \prime}, h_{C}^{\prime \prime}, h_{B}^{\prime \prime}\right)
$$

Lemma 4.21 shows that the height function behaves multiplicatively on a tensor product of bimodules, $H\left(\mathcal{E}, h_{A}, h_{C}\right)=H\left(\mathcal{E}^{\prime}, h_{A}^{\prime}, h_{B}^{\prime}\right) H\left(\mathcal{E}^{\prime \prime}, h_{B}^{\prime \prime}, h_{C}^{\prime \prime}\right)$, so that these two expressions agree.

Thus, the (non-normalized) height (4.13) of hermitian bimodules in $\mathcal{H}(\mathbb{K}, Z)$ makes the spaces $X_{\left(A, \mathcal{O}_{A}\right)}^{n c}$ (of which $\mathcal{A}_{\mathcal{H}(\mathbb{K})}^{(1)}$ represents the algebra of functions) dynamical through the time evolution $\alpha_{t}$. Notice that this dynamics, generated by a ratio of volumes on the two sides of a hermitian bimodule, is very similar to the time evolution generated by the ratio of volumes of two commensurable lattices in the case of the quantum statistical mechanical system of [11]. We can similarly define a time evolution on the algebra of 2-morphisms $\mathcal{A}_{\mathcal{H}(\mathbb{K}, Z)}^{(2)}$ of $\mathcal{H}(\mathbb{K}, Z)$, using a notion of relative height. 
Definition 4.23. The relative height $H(\phi)$ of a 2 -morphism $\phi:\left(\mathcal{E}, h_{A}, h_{B}\right) \rightarrow$ $\left(\mathcal{E}^{\prime}, h_{A}^{\prime}, h_{B}^{\prime}\right)$ in $\mathcal{H}(\mathbb{K}, Z)$ is defined as

$$
H(\phi):=\frac{H\left(\tilde{\mathcal{E}}, \tilde{h}_{A}, \tilde{h}_{B}\right)}{H\left(\mathcal{E}, h_{A}, h_{B}\right)} .
$$

Lemma 4.24. We can write (4.16) equivalently in the form

$$
H(\phi)=\frac{\inf _{\left\{y_{r}\right\} \in \mathcal{B}_{B}(\tilde{\mathcal{E}}) \backslash \mathcal{B}_{B}(\mathcal{E})} \prod_{\sigma} \prod_{r} \tilde{\sigma}\left(\tilde{h}_{B}\left(y_{r}, y_{r}\right)\right)}{\inf _{\left\{x_{k}\right\} \in \mathcal{B}_{A}(\tilde{\mathcal{E}}) \backslash \mathcal{B}_{A}(\mathcal{E})} \prod_{\sigma} \prod_{k} \tilde{\sigma}\left(\tilde{h}_{A}\left(x_{k}, x_{k}\right)\right)} .
$$

Proof. Let us recall Definition 4.20, which tells us that,

$$
H\left(\mathcal{E}, h_{A}, h_{B}\right):=\prod_{\sigma} \frac{H_{h_{B}, \sigma}(\mathcal{E})}{H_{h_{A}, \sigma}(\mathcal{E})}
$$

where

$$
\begin{aligned}
& H_{h_{A}, \sigma}(\mathcal{E}):=\inf _{\left\{x_{i}\right\} \in \mathcal{B}_{A}(\mathcal{E})} \prod_{i} \tilde{\sigma}\left(h_{A}\left(x_{i}, x_{i}\right)\right) \\
& H_{h_{B}, \sigma}(\mathcal{E}):=\inf _{\left\{y_{j}\right\} \in \mathcal{B}_{B}(\mathcal{E})} \prod_{j} \tilde{\sigma}\left(h_{B}\left(y_{j}, y_{j}\right)\right) .
\end{aligned}
$$

Then, by definition, we get

$$
\begin{aligned}
H(\phi):= & \frac{H\left(\tilde{\mathcal{E}}, \tilde{h}_{A}, \tilde{h}_{B}\right)}{H\left(\mathcal{E}, h_{A}, h_{B}\right)} \\
= & \frac{\prod_{\sigma} \frac{H_{\tilde{h}_{B}, \sigma}(\mathcal{E})}{H_{\tilde{h}_{A}, \sigma}(\mathcal{E})}}{\prod_{\sigma} \frac{H_{h_{B}, \sigma}(\mathcal{E})}{H_{h_{A}, \sigma}(\mathcal{E})}} \\
= & \frac{\left(\inf _{\left\{\tilde{y}_{r}\right\} \in \mathcal{B}_{B}(\tilde{\mathcal{E}})} \prod_{\sigma} \prod_{r} \tilde{\sigma}\left(\tilde{h}_{B}\left(\tilde{y}_{r}, \tilde{y}_{r}\right)\right)\right)\left(\inf _{\left\{x_{i}\right\} \in \mathcal{B}_{A}(\mathcal{E})} \prod_{\sigma} \prod_{i} \tilde{\sigma}\left(h_{A}\left(x_{i}, x_{i}\right)\right)\right)}{\left(\inf _{\left\{\tilde{x}_{k}\right\} \in \mathcal{B}_{A}(\tilde{\mathcal{E}})} \prod_{\sigma} \prod_{k} \tilde{\sigma}\left(\tilde{h}_{A}\left(\tilde{x}_{k}, \tilde{x}_{k}\right)\right)\right)\left(\inf _{\left\{y_{j}\right\} \in \mathcal{B}_{B}(\mathcal{E})} \prod_{\sigma} \prod_{j} \tilde{\sigma}\left(h_{B}\left(y_{j}, y_{j}\right)\right)\right)} \\
= & \frac{\left(\inf _{\left\{\tilde{y}_{r}\right\} \in \mathcal{B}_{B}(\tilde{\mathcal{E}})} \prod_{\sigma} \prod_{r} \tilde{\sigma}\left(\tilde{h}_{B}\left(\tilde{y}_{r}, \tilde{y}_{r}\right)\right)\right)\left(\inf _{\left\{x_{i}\right\} \in \mathcal{B}_{A}(\mathcal{E})} \prod_{\sigma} \prod_{i} \tilde{\sigma}\left(\tilde{h}_{A}\left(\phi\left(x_{i}\right), \phi\left(x_{i}\right)\right)\right)\right)}{\left(\inf _{\left\{\tilde{x}_{k}\right\} \in \mathcal{B}_{A}(\tilde{\mathcal{E}})} \prod_{\sigma} \prod_{k} \tilde{\sigma}\left(\tilde{h}_{A}\left(\tilde{x}_{k}, \tilde{x}_{k}\right)\right)\right)\left(\inf _{\left\{y_{j}\right\} \in \mathcal{B}_{B}(\mathcal{E})} \prod_{\sigma} \prod_{j} \tilde{\sigma}\left(\tilde{h}_{B}\left(\phi\left(y_{j}\right), \phi\left(y_{j}\right)\right)\right)\right)}
\end{aligned}
$$

Lemma 4.25. The relative non-normalized height $H(\phi)$ of (4.16) determines a time evolution on the algebra $\mathcal{A}_{\mathcal{H}(\mathbb{K}, Z)}^{(2)}$ of 2-morphisms that is compatible with both vertical and horizontal composition.

Proof. We define the time evolution as $\alpha_{t}(f)(\phi)=H(\phi)^{i t} f(\phi)$. To verify compatibility with the two product structures of $\mathcal{A}_{\mathcal{H}(\mathbb{K}, Z)}^{(2)}$, consider a vertical composition $\phi^{\prime \prime} \circ \phi^{\prime}$ with $\phi^{\prime}:\left(\mathcal{E}, h_{A}, h_{B}\right) \rightarrow\left(\mathcal{E}^{\prime}, h_{A}^{\prime}, h_{B}^{\prime}\right)$ and $\phi^{\prime \prime}:\left(\mathcal{E}^{\prime}, h_{A}^{\prime}, h_{B}^{\prime}\right) \rightarrow\left(\mathcal{E}^{\prime \prime}, h_{A}^{\prime \prime}, h_{B}^{\prime \prime}\right)$. By (4.16) we have $H\left(\phi^{\prime \prime} \circ \phi^{\prime}\right)=H\left(\phi^{\prime}\right) H\left(\phi^{\prime \prime}\right)$, hence we have

$$
\alpha_{t}\left(f_{1} \circ f_{2}\right)(\phi)=H(\phi)^{i t} \sum_{\phi=\phi^{\prime \prime} \circ \phi^{\prime}} f_{1}\left(\phi^{\prime}\right) f_{2}\left(\phi^{\prime \prime}\right)=\sum_{\phi=\phi^{\prime \prime} \circ \phi^{\prime}} \alpha_{t}\left(f_{1}\right)\left(\phi^{\prime}\right) \alpha_{t}\left(f_{2}\right)\left(\phi^{\prime \prime}\right) .
$$


In the case of a horizontal composition of $\phi^{\prime}:\left(\mathcal{E}, h_{A}, h_{B}\right) \rightarrow\left(\tilde{\mathcal{E}}, \tilde{h}_{A}, \tilde{h}_{B}\right)$ with $\left(\mathcal{E}, h_{A}, h_{B}\right)$ the tensor product of $\left(\mathcal{E}^{\prime}, h_{A}^{\prime}, h_{B}^{\prime}\right)$ and $\left(\mathcal{E}^{\prime \prime}, h_{A}^{\prime \prime}, h_{B}^{\prime \prime}\right)$ and $\left(\tilde{\mathcal{E}}, \tilde{h}_{A}, \tilde{h}_{B}\right)$ the tensor product of $\left(\tilde{\mathcal{E}}^{\prime}, \tilde{h}_{A}^{\prime}, \tilde{h}_{B}^{\prime}\right)$ and $\left(\tilde{\mathcal{E}}^{\prime \prime}, \tilde{h}_{A}^{\prime \prime}, \tilde{h}_{B}^{\prime \prime}\right)$, as in Lemma 4.22, with $\phi=\phi^{\prime \prime} \bullet \phi^{\prime}$, by Lemma 4.24 we have

$$
\begin{gathered}
\alpha_{t}\left(f_{1} \bullet f_{2}\right)(\phi)=\left(\frac{H\left(\mathcal{E}, h_{A}, h_{B}\right)}{H\left(\tilde{\mathcal{E}}, \tilde{h}_{A}, \tilde{h}_{B}\right)}\right)^{i t} \sum_{\phi=\phi^{\prime \prime} \bullet \phi^{\prime}} f_{1}\left(\phi^{\prime}\right) f_{2}\left(\phi^{\prime \prime}\right) \\
=\sum_{\phi=\phi^{\prime \prime} \bullet \phi^{\prime}}\left(\frac{H\left(\mathcal{E}^{\prime}, h_{A}^{\prime}, h_{B}^{\prime}\right) H\left(\mathcal{E}^{\prime \prime}, h_{A}^{\prime \prime}, h_{B}^{\prime \prime}\right)}{H\left(\tilde{\mathcal{E}}^{\prime}, \tilde{h}_{A}^{\prime}, \tilde{h}_{B}^{\prime}\right) H\left(\tilde{\mathcal{E}}^{\prime \prime}, \tilde{h}_{A}^{\prime \prime}, h_{B}^{\prime \prime}\right)}\right)^{i t} f_{1}\left(\phi^{\prime}\right) f_{2}\left(\phi^{\prime \prime}\right)=\sum_{\phi=\phi^{\prime \prime} \bullet \phi^{\prime}} \alpha_{t}\left(f_{1}\right)\left(\phi^{\prime}\right) \alpha_{t}\left(f_{2}\right)\left(\phi^{\prime \prime}\right) .
\end{gathered}
$$

4.11. Partition function and height zeta function. We can consider subsystems of the algebras with time evolution discussed above, where the Hamiltonian $\mathfrak{H}$ generating the time evolution has an associated partition function $Z(\beta)=\operatorname{Tr}\left(e^{-\beta \mathfrak{H}}\right)$ that defines a height zeta function. To avoid obvious sources of infinite multiplicities, we can restrict to the subalgebra $\mathcal{A}_{\mathcal{H}_{A}^{L}(\mathbb{K})}^{(1)}$ of $\mathcal{A}_{\mathcal{H}(\mathbb{K}, Z)}^{(1)}$ of functions supported on the subset $\mathcal{H}^{L}(\mathbb{K}, Z)$ of $\mathcal{H}(\mathbb{K}, Z)$ consisting of $A$ - $A$ bimodules $\left(\mathcal{E}, h_{A}^{L}, h_{A}^{R}\right)$ with $H^{L}\left(\mathcal{E}, h_{A}^{L}, h_{A}^{R}\right)=\prod_{\sigma} H_{h_{A}^{L}, \sigma}(\mathcal{E})=1$, for which $H\left(\mathcal{E}, h_{A}^{L}, h^{R}\right)=H^{R}\left(\mathcal{E}, h_{A}^{L}, h^{R}\right)=$ $\prod_{\sigma} H_{h_{A}^{R}, \sigma}(\mathcal{E})$. This is indeed a subalgebra since for bimodules in $\mathcal{H}(\mathbb{K}, Z)$ we have $H_{A}^{L}\left(\mathcal{E} \otimes_{A} \mathcal{F}, h_{A}^{L}, h_{A}^{R}\right)=H_{A}^{L}\left(\mathcal{E}, h_{A}^{L,{ }^{\prime}}, h_{A}^{R,{ }^{\prime}}\right) H_{A}^{L}\left(\mathcal{F}, h_{A}^{L,{ }^{\prime \prime}}, h_{A}^{R,{ }^{\prime \prime}}\right)$ and both factors are equal to one if both $\mathcal{E}$ and $\mathcal{F}$ are in $\mathcal{H}_{A}^{L}(\mathbb{K})$, so the tensor product $\mathcal{E} \otimes_{B} \mathcal{F}$ is also in $\mathcal{H}_{A}^{L}(\mathbb{K})$. Thus, we consider the subsystem $\left(\mathcal{A}_{\mathcal{H}_{A}^{L}(\mathbb{K})}^{(1)}, \alpha_{t}\right)$ with the same time evolution discussed above. The Hamiltonian generating this time evolution is given by

$$
\mathfrak{H} f\left(\mathcal{E}, h_{A}^{L}, h_{A}^{R}\right)=\log H_{A}^{R}\left(\mathcal{E}, h_{A}^{L}, h_{A}^{R}\right) f\left(\mathcal{E}, h_{A}^{L}, h_{A}^{R}\right),
$$

with $\alpha_{t}(f)=e^{-i t H} f e^{i t H}$, for functions $f \in \mathcal{A}_{\mathcal{H}_{A}^{L}(\mathbb{K})}^{(1)}$ seen as acting on the Hilbert space $\ell^{2}\left(\mathcal{H}_{A}^{L}(\mathbb{K})\right)$. The partition function is then of the form

$$
Z(\beta)=\operatorname{Tr}\left(e^{-\beta \mathfrak{H}}\right)=\sum_{\left(\mathcal{E}, h_{A}^{L}, h_{A}^{R}\right) \in \mathcal{H}_{A}^{L}(\mathbb{K})} H_{B}\left(\mathcal{E}, h_{A}^{L}, h_{A}^{R}\right)^{-\beta},
$$

which gives the height zeta function for our definition of height. The question of whether this height zeta function is convergent for sufficiently large $\beta>0$ corresponds then to the question of whether there are finitely many "points" $\left(\mathcal{E}, h_{A}, h_{B}\right)$ of bounded height $H_{B}\left(\mathcal{E}, h_{A}^{L}, h_{A}^{R}\right) \leq \kappa$ (with $H_{A}\left(\mathcal{E}, h_{A}^{L}, h_{A}^{R}\right)=1$ ), which corresponds to the property that the spectrum of the Hamiltonian $\mathfrak{H}$ has finite multiplicities, and the question of the asymptotic behavior of this height bound. We will return to discuss a simpler version of this height zeta function after introducing a version of the height function based on the Hattori-Stallings rank in $\$ 4.12$. 
4.12. Limitations of this notion of height. The height function as described in the previous subsections provides us with a viable analog of the corresponding notion used in [6], which in turn extends the commutative case where the height is computed in the form of a volume (or normalized volume).

As we discussed in the previous subsections, using this notion of height, however forces us to make some strong assumptions in order to ensure the multiplicative behavior with respect to the composition (tensor product) of bimodules. Moreover, it is clear that the ad hoc solution that we adopted to circumvent this problem is specific to the finite dimensional case (hence to noncommutative arithmetic curves) and does not generalize to higher dimensional cases.

Thus, it seems preferable to rethink the notion of height in this noncommutative context in such a way that the multiplicative property under composition of bimodules would be naturally satisfied, in a way that does not depend on special conditions on the bimodules, and that extends to the case of algebras that are not finite dimensional, with Hilbert bimodules of finite type.

The prototype example of an invariant of bimodules of finite type that satisfies the multiplicative property is the Jones index (in the case of simple algebras). We will use this as a model for the properties that a desirable height function should satisfy.

As a heuristic justification for making this change of perspective, and proposing a different choice of height function, it is useful to keep in mind that in the usual arithmetic geometry context the height function comes in two flavors, a "logarithmic form" and an "exponential form" (see [5]). The latter is the one that gives rise to the volume description of the height presented in (4.2) and generalized in (4.9). The first is usually taken to be just the logarithm of the exponential one, $\mathfrak{h}=\log H$. This logarithmic form implies that if $H$ behaves like a volume, which scales with the size $\lambda$ in the form vol $\sim \lambda^{\operatorname{dim}}$, then the $\mathfrak{h}=\log H$ would be proportional to the dimension. What we propose here is not the usual form $\mathfrak{h}=\log H$ of the height, with $H$ as in (4.2), (4.9), but rather the use of the dimension itself, in the form of the Hattori-Stallings rank element already discussed in \$4.6.

If we define

$$
\mathfrak{h}_{A}(\mathcal{E}):=r_{A}(\mathcal{E}) \in H H_{0}(A)
$$

the Hattori-Stallings rank, and

$$
\mathfrak{h}_{A, \sigma}\left(\mathcal{E}, h_{A}, h_{B}\right):=\tilde{\sigma}\left(r_{A}(\mathcal{E})\right)
$$

with an embedding $\tilde{\sigma}$ of $Z(A)$ that extends the embedding $\sigma$ of $\mathbb{K}$, then we obtain a better notion of height on bimodules that does not require restricting the category of bimodules as in Definition 4.17.

A further advantage of working with this definition of height $\mathfrak{h}_{A}(\mathcal{E}):=r_{A}(\mathcal{E})$ is that the Hattori-Stallings rank is independent of the generators $\left\{x_{i}\right\}$ of the projective module $\mathcal{E}$. Indeed, the rank element can be computed using a choice of $\left\{x_{i}\right\}$ and of a dual basis $\left\{\xi_{i}\right\}$ of $\mathcal{E}^{\vee}$ by $r_{A}(\mathcal{E})=\sum_{i} \xi_{i}\left(x_{i}\right)$, but the result is independent of the choice of $\left\{x_{i}\right\}$ and $\left\{\xi_{i}\right\}$. Thus, we do not have to optimize over a choice of the basis, unlike in the case of the height (4.9) previously discussed. 
Proposition 4.26. Consider arithmetic hermitian bimodules $\left(\mathcal{E}, h_{A}, h_{B}\right)$ in the category ${ }_{\left(A, \mathcal{O}_{A}\right)} \mathcal{H}_{\left(B, \mathcal{O}_{B}\right)}$ with the height defined as

$$
\mathfrak{h}\left(\mathcal{E}, h_{A}, h_{B}\right)=\prod_{\sigma} \frac{\sigma\left(r_{B}(\mathcal{E})\right.}{\sigma\left(r_{A}(\mathcal{E})\right.},
$$

where $\sigma$ ranges over the archimedean embeddings. Let $\mathcal{A}_{\mathcal{H}(\mathbb{K})}^{(1)}$ be the convolution algebra of finitely supported functions over the set of bimodules in ${ }_{\left(A, \mathcal{O}_{A}\right)} \mathcal{H}_{\left(B, \mathcal{O}_{B}\right)}$ with the convolution product as in (4.15). Then setting

$$
\alpha_{t}(f)\left(\mathcal{E}, h_{A}, h_{B}\right)=\mathfrak{h}\left(\mathcal{E}, h_{A}, h_{B}\right)^{i t} f\left(\mathcal{E}, h_{A}, h_{B}\right)
$$

determines a time evolution on $\mathcal{A}_{\mathcal{H}(\mathbb{K})}^{(1)}$.

Proof. As above, we can compute the Hattori-Stallings rank as $r_{A}(\mathcal{E})=\sum_{i} \xi_{i}\left(x_{i}\right)$, for a choice of generators $\left\{x_{i}\right\}$ of $\mathcal{E}$ and a dual set $\left\{\xi_{i}\right\}$ in $\mathcal{E}^{\vee}$. In particular, for $\xi_{i}=h_{A}\left(\cdot, x_{i}\right)$, we obtain $r_{A}(\mathcal{E})=\sum_{i} h_{A}\left(x_{i}, x_{i}\right)$. Thus, we are replacing the volume $\prod_{i} h_{A}\left(x_{i}, x_{i}\right)$ of our previous definition of height (4.9) with the trace $\sum_{i} h_{A}\left(x_{i}, x_{i}\right)$. This sum is in $Z(A)=\oplus_{i} \mathbb{L}_{i}$ for some field extensions $\mathbb{L}_{i}$ of the number field $\mathbb{K}$. Given an archimedean embedding, $\sigma: \mathbb{K} \hookrightarrow \mathbb{R}$ or $\mathbb{C}$, extended to an embedding $\tilde{\sigma}$ of the $\mathbb{L}_{i}$, we obtain $\tilde{\sigma}\left(r_{A}(\mathcal{E})\right) \in \mathbb{R}$ or $\mathbb{C}$. Since the $h_{A}\left(x_{i}, x_{i}\right)$ are positive elements of $A$, the value of $\tilde{\sigma}\left(r_{A}(\mathcal{E})\right)$ is actually in $\mathbb{R}_{+}^{*}$. Thus, we have $\mathfrak{h}\left(\mathcal{E}, h_{A}, h_{B}\right)^{i t} \in U(1)$ for $t \in \mathbb{R}$ defining the time evolution. We need to know that $\sigma_{t}\left(f_{1} \star f_{2}\right)=\sigma_{t}\left(f_{1}\right) \star \sigma_{t}\left(f_{2}\right)$ with respect to the product (4.15). This follows by verifying that

$$
\mathfrak{h}\left(\mathcal{E} \otimes_{B} \mathcal{F}, h_{A}, h_{C}\right)=\mathfrak{h}\left(\mathcal{E}, h_{A}^{\prime}, h_{B}^{\prime}\right) \cdot \mathfrak{h}\left(\mathcal{F}, h_{B}^{\prime \prime}, h_{C}^{\prime \prime}\right),
$$

for a tensor product of bimodules $\mathcal{E} \otimes_{B} \mathcal{F}$, with the hermitian structures $h_{A}$ and $h_{C}$ as in Lemma 4.16. This is indeed the case because we have

$$
\begin{gathered}
r_{A}\left(\mathcal{E} \otimes_{B} \mathcal{F}\right)=\sum_{i j} h_{A}^{\prime}\left(x_{i}^{\prime} h_{B}^{\prime \prime}\left(x_{j}^{\prime \prime}, x_{j}^{\prime \prime}\right), x_{i}\right)=\sum_{i} h_{A}^{\prime}\left(x_{i}^{\prime} \cdot r_{B}(\mathcal{F}), x_{i}^{\prime}\right) \\
r_{C}\left(\mathcal{E} \otimes_{B} \mathcal{F}\right)=\sum_{\ell . r} h_{C}^{\prime \prime}\left(u_{\ell}^{\prime \prime}, h_{B}^{\prime}\left(u_{r}^{\prime}, u_{r}^{\prime}\right) u_{\ell}^{\prime \prime}\right)=\sum_{\ell} h_{C}^{\prime \prime}\left(u_{\ell}^{\prime \prime}, r_{B}(\mathcal{E}) \cdot u_{\ell}^{\prime \prime}\right),
\end{gathered}
$$

where $r_{B}(\mathcal{F})$ and $r_{B}(\mathcal{E})$ are in $Z(B)$. For a fixed embedding $\sigma: \mathbb{K} \hookrightarrow \mathbb{R}$ or $\mathbb{C}$, and an embedding $\tilde{\sigma}$ of the field extensions in $Z(A), Z(B)$, and $Z(C)$, that restricts to $\sigma$ on the subfield $\mathbb{K}$, the hermitian forms $\left(h_{A, \sigma}^{\prime}, h_{B, \sigma}^{\prime}\right)$ on $\mathcal{E}_{\sigma}$ and $\left(h_{B, \sigma}^{\prime \prime}, h_{C, \sigma}^{\prime \prime}\right)$ on $\mathcal{F}_{\sigma}$, with values in $A_{\sigma}$ and $B_{\sigma}$, respectively $B_{\sigma}$ and $C_{\sigma}$, give

$$
\begin{gathered}
\sigma\left(r_{A}(\mathcal{E})\right)=\sum_{i} h_{A, \sigma}^{\prime}\left(x_{i}^{\prime} \cdot \sigma\left(r_{B}(\mathcal{F})\right), x_{i}^{\prime}\right)=\sigma\left(r_{B}(\mathcal{F})\right) \cdot \sigma\left(r_{A}(\mathcal{E})\right), \\
\sigma\left(r_{C}\left(\mathcal{E} \otimes_{B} \mathcal{F}\right)\right)=\sum_{\ell} h_{C, \sigma}^{\prime \prime}\left(u_{\ell}^{\prime \prime}, \sigma\left(r_{B}(\mathcal{E})\right) \cdot u_{\ell}^{\prime \prime}\right)=\sigma\left(r_{C}(\mathcal{F})\right) \cdot \sigma\left(r_{B}(\mathcal{E})\right),
\end{gathered}
$$

so that we obtain

$$
\mathfrak{h}\left(\mathcal{E} \otimes_{B} \mathcal{F}, h_{A}, h_{C}\right)=\prod_{\sigma} \frac{\sigma\left(r_{C}(\mathcal{F})\right)}{\sigma\left(r_{B}(\mathcal{F})\right)} \frac{\sigma\left(r_{B}(\mathcal{E})\right)}{\sigma\left(r_{A}(\mathcal{E})\right)} .
$$

This completes the proof. 
The Hattori-Stallings rank is known to agree with the Jones index, [23], [24], [57], in the cases we will be considering in $\$ 5$, so this discussion justifies and introduces the setting for higher dimensional arithmetic noncommutative spaces that we will be discussing below in $\$ 5$.

4.13. Height zeta function with the Hattori-Stallings rank. We can consider again a subsystem with a partition function that provides a height zeta function, for the case of the height $\mathfrak{h}\left(\mathcal{E}, h_{A}, h_{B}\right)$. As we discussed in \$4.11, in order to avoid infinite multiplicities in the spectrum of the Hamiltonian that generates the time evolution, we restrict to the subcategory $\mathcal{H}_{A}^{L}(\mathbb{K})$ of $\mathcal{H}(\mathbb{K}) A$-A bimodules $\left(\mathcal{E}, h_{A}^{L}, h_{A}^{R}\right)$ and with rank (with respect to the structure of left $A$-module) fixed to be $r_{A}^{L}(\mathcal{E})=$ 1. This determines a subalgebra $\mathcal{A}_{\mathcal{H}_{A}^{L}(\mathbb{K})}^{(1)}$ of the algebra of functions on $\mathcal{H}(\mathbb{K})$ with the convolution product corresponding to the tensor product of bimodules, and the Hamiltonian that generates the time evolution, for the algebra $\mathcal{A}_{\mathcal{H}_{A}^{L}(\mathbb{K})}^{(1)}$ acting on the Hilbert space $\ell^{1}\left(\mathcal{H}_{A}^{L}(\mathbb{K})\right)$, is given by

$$
\mathfrak{H} f\left(\mathcal{E}, h_{A}^{L}, h_{A}^{R}\right)=\log \left(\prod_{\sigma} r_{A, \sigma}^{R}(\mathcal{E})\right) f\left(\mathcal{E}, h_{A}^{L}, h_{A}^{R}\right) .
$$

and with partition function

$$
Z(\beta)=\operatorname{Tr}\left(e^{-\beta \mathfrak{H}}\right)=\sum_{\left(\mathcal{E}, h_{A}^{L}, h_{A}^{R}\right) \in \mathcal{H}_{A}^{L}(\mathbb{K})}\left(\prod_{\sigma} r_{A, \sigma}^{R}(\mathcal{E})\right)^{-\beta} .
$$

The multiplicities of the eigenvalues of the Hamiltonian $\mathfrak{H}$, which determine if the series defining the partition function is convergent for sufficiently large $\beta>0$, count the number of $A$-A bimodules $\left(\mathcal{E}, h_{A}^{L}, h_{A}^{R}\right)$ with $r_{A}^{L}(\mathcal{E})=1$ and with fixed $r_{A}^{R}(\mathcal{E})=r$. For a finite dimensional semisimple algebra $A=\oplus_{i} M_{n_{i}}\left(D_{i}\right)$ with division algebras $D_{i}$ over $\mathbb{K}$, we can consider bimodules for the form $D_{i} \otimes_{\mathbb{K}} \oplus_{j} D_{j}^{n_{j} N_{j}}$ such that $\sum_{j} n_{j} N_{j}=r$, which are counted by the number of these combinations, the number of solutions $N_{j} \geq 0$ of the relation above with $r$ and the $n_{i}$ given.

4.14. Non-archimedean places. So far we only considered the archimedean component of the height function, associated to the archimedean places $\sigma: \mathbb{K} \hookrightarrow \mathbb{K}_{\sigma}$ of the number field $\mathbb{K}$. The height function also has components associated to the non-archimedean places. For example, one sees clearly that the usual definition of the (archimedean) height function for points in projective spaces, $H_{\sigma}(x)=\max _{i}\left|x_{i}\right|_{\sigma}$, for $x=\left(x_{1}: \cdots: x_{n}\right)$ is only well defined when the non-archimedean places are taken into account in a product over all valuations, $H(x)=\prod_{v} \max _{i}\left|x_{i}\right|_{v}$, since by the product formula $H(\lambda x)=H(x)$ for $\lambda \in \mathbb{G}_{m}$. This issue does not arise in an affine setting, but it is still more natural to regard the height as a product over all archimedean and non-archimedean valuations.

We discuss here briefly how to extend the height functions discussed in the previous subsection to the non-archimedean places. 
Since we are working in a noncommutative setting, we recall briefly how one thinks of valuations in the context of finite dimensional algebras. Given a semisimple finite dimensional algebra $A=\oplus_{i} M_{n_{i}}\left(D_{i}\right)$ over a number field $\mathbb{K}$, let $\mathbb{K}_{v}$ denote the completions at the non-archimedean valuations. These are extensions of the p-adic fields $\mathbb{Q}_{p}$ for primes $p$. We write $A_{v}=A \otimes_{\mathbb{K}} \mathbb{K}_{v}$ for the corresponding algebras over $\mathbb{K}_{v}$. The fields $\mathbb{K}_{v}$ are henselian, namely a valuation on $\mathbb{K}_{v}$ has a unique extension to each field algebraic over $\mathbb{K}_{v}$, [49]. Let $D$ be a division algebra over $\mathbb{K}_{v}$ and let $Z(D)=\mathbb{F}$, an extension of $\mathbb{K}_{v}$. There are different possible ways of defining valuations on a division algebra, summarized in [56]. We take here the following definition: a valuation on a division algebra $D$ is a function $v: D^{*} \rightarrow \Gamma$, where $\Gamma$ is a totally ordered abelian group such that

$$
v(a b)=v(a)+v(b) \quad \text { and } \quad v(a+b) \geq \min \{v(a), v(b)\} \text { for } b \neq-a .
$$

Theorem 2.1 of [56] shows that, for a division algebra $D$ with $Z(D)=\mathbb{F}$ and $\operatorname{dim}_{\mathbb{F}}(D)<\infty$, a valuation $v$ on $\mathbb{F}$ extends (uniquely) to a valuation on $D$ if and only if $v$ extends uniquely to any field $\mathbb{L}$ with $\mathbb{F} \subset \mathbb{L} \subset D$. In particular, if $\mathbb{F}$ is henselian, then a valuation on $\mathbb{F}$ extends uinquely to a valuation on any finite dimensional division algebra $D$ with $Z(D)=\mathbb{F}$. Thus, the non-archimedean valuation $v$ on $\mathbb{K}_{v}$, extends uniquely to a division algebra over $\mathbb{K}_{v}$. Moreover, a valuation on a division algebra $D$ over $\mathbb{K}_{v}$ restricts to compatible valuations on subfields of $D$ hence they all arise in this way. Thus, it suffices to consider the non-archimedean places of $\mathbb{K}$ with the corresponding valuations $v$ and the associated completions $\mathbb{K}_{v}$ to account for all valuations on division algebras as well.

Consider then arithmetic bimodules $\left(\mathcal{E}, h_{A}, h_{B}\right)$ with hermitian structures $h_{A}, h_{B}$. Consider the elements $h_{A}\left(x_{i}, x_{i}\right) \in A$ and $h_{B}\left(y_{j}, y_{j}\right) \in B$, for sets of generators $\left\{x_{i}\right\}$ and $\left\{y_{j}\right\}$. In the case of the height function $H\left(\mathcal{E}, h_{A}, h_{B}\right)$ of (4.9), and bimodules $\left(\mathcal{E}, h_{A}, h_{B}\right)$ in the class $\mathcal{H}(\mathbb{K}, Z)$ of Definition 4.17, we have $h_{A}\left(x_{i}, x_{i}\right) \in Z(A)=Z$ and $h_{B}\left(y_{j}, y_{j}\right) \in Z(B)=Z$ and we can compose these elements with the valuation $v$ (the unique extension to the $\mathbb{L}_{i}$ of the valuation $v$ on $\mathbb{K}_{v}$ ). Thus, we can define $H_{v}\left(\mathcal{E}, h_{A}, h_{B}\right)$ as in (4.9) in the form

$$
H_{v}\left(\mathcal{E}, h_{A}, h_{B}\right):=\frac{\inf _{\left\{y_{j}\right\} \in \mathcal{B}_{B}(\mathcal{E})}\left|\prod_{j} h_{B}\left(y_{j}, y_{j}\right)\right|_{v}}{\inf _{\left\{x_{i}\right\} \in \mathcal{B}_{A}(\mathcal{E})}\left|\prod_{i} h_{A}\left(x_{i}, x_{i}\right)\right|_{v}},
$$

where a shortest basis is used to evaluate these "volumes". We then set

$$
H\left(\mathcal{E}, h_{A}, h_{B}\right):=\prod_{v} H_{v}\left(\mathcal{E}, h_{A}, h_{B}\right) \cdot \prod_{\sigma} H_{\sigma}\left(\mathcal{E}, h_{A}, h_{B}\right),
$$

with $v$ ranging over the non-archimedean places of $\mathbb{K}$ and $\sigma$ over the archimedean places. Note that because at each place a (different) shortest basis $\left\{x_{i}\right\}$ is chosen that minimizes the corresponding "volume" $\left|\prod_{i} h_{A}\left(x_{i}, x_{i}\right)\right|_{v}$, the product over places considered above is not subject to the product formula $\prod_{v}|\lambda|_{v} \cdot \prod_{\sigma}|\lambda|_{\sigma}=1$ for all $\lambda \in \mathbb{L}$ (a number field), for the product over all archimedean and non-archimedean valuations of $\mathbb{L}$. The time evolutions described in the previous subsections extend to these height functions that include the contributions of the non-archimedean places. 
In the case of the height function defined using the rank element, and for any arithmetic hermitian bimodule, however, we cannot just define the height $\mathfrak{h}\left(\mathcal{E}, h_{A}, h_{B}\right)$ in the same way as above, because for $r_{A}(\mathcal{E}) \in \mathbb{L}$ the product formula would give

$$
\prod_{v}\left|r_{A}(\mathcal{E})\right|_{v} \cdot \prod_{\sigma}\left|r_{A}(\mathcal{E})\right|_{\sigma}=1
$$

This means that, in one defines the non-archimedean part of the height as in the previous case, then the non-archimedean part of the height would simply be equivalent to the archimedean part by the relation

$$
\mathfrak{h}^{\text {nar }}\left(\mathcal{E}, h_{A}, h_{B}\right)=\prod_{v} \frac{\left|r_{B}(\mathcal{E})\right|_{v}}{\left|r_{A}(\mathcal{E})\right|_{v}}=\mathfrak{h}^{a r}\left(\mathcal{E}, h_{A}, h_{B}\right)^{-1}=\prod_{\sigma} \frac{\left|r_{A}(\mathcal{E})\right|_{\sigma}}{\left|r_{B}(\mathcal{E})\right|_{\sigma}},
$$

up to a change of direction of the time evolution they induce. Thus, in the case of the height function defined by the Hattori-Stallings rank, one can restrict to only considering the archimedean part.

There is another possible way to extend the Hattori-Stallings rank to a height function with a non-archimedean components, so that the product over all archimedean and non-archimedean places is non-trivial. If we view the archimedean part as

$$
\prod_{\sigma}\left|r_{A}(\mathcal{E})\right|_{\sigma}=\prod_{\sigma} \sum_{i} h_{A, \sigma}\left(x_{i}, x_{i}\right)=\prod_{\sigma} \sum_{i}\left\|x_{i}\right\|_{A, \sigma}^{2},
$$

then it is natural to define

$$
\mathfrak{h}^{a r}\left(\mathcal{E}, h_{A}, h_{B}\right):=\prod_{\sigma} \frac{\left|r_{B}(\mathcal{E})\right|_{\sigma}^{1 / 2}}{\left|r_{A}(\mathcal{E})\right|_{\sigma}^{1 / 2}}=\prod_{\sigma} \frac{\left(\sum_{j}\left\|y_{j}\right\|_{B, \sigma}^{2}\right)^{1 / 2}}{\left(\sum_{i}\left\|x_{i}\right\|_{A, \sigma}^{2}\right)^{1 / 2}}
$$

By comparison with the usual definition of the height of points $x=\left(x_{1}, \ldots, x_{n}\right) \in \overline{\mathbb{Q}}^{n}$ in the form

$$
H(x)=H^{n a r}(x) H^{a r}(x)=\prod_{v}\left(\max _{i}\left|x_{i}\right|_{v}\right) \cdot \prod_{\sigma}\left(\sum_{i}\left|x_{i}\right|_{\sigma}^{2}\right)^{1 / 2},
$$

one can choose to set

$$
\mathfrak{h}\left(\mathcal{E}, h_{A}, h_{B}\right)=\mathfrak{h}^{\operatorname{nar}}\left(\mathcal{E}, h_{A}, h_{B}\right) \mathfrak{h}^{\text {ar }}\left(\mathcal{E}, h_{A}, h_{B}\right)
$$

where the archimedean part is defined as above and

$$
\mathfrak{h}^{\text {nar }}\left(\mathcal{E}, h_{A}, h_{B}\right):=\prod_{v} \frac{\inf _{\left\{y_{j}\right\} \in \mathcal{B}_{B}(\mathcal{E})} \max _{j}\left|h_{B}\left(y_{j}, y_{j}\right)\right|_{v}}{\inf _{\left\{x_{i}\right\} \in \mathcal{B}_{A}(\mathcal{E})} \max _{i}\left|h_{A}\left(x_{i}, x_{i}\right)\right|_{v}} .
$$

Notice, however, that with this choice of non-archimedean part of the height, we have reintroduced the problem of optimizing over the choice of a basis and also the problem of the compatibility of the height with the tensor product of bimodules, which forces us then to restrict the category of bimodules to the $\mathcal{H}(\mathbb{K}, Z)$ of Definition 4.17, both problems that the use of the Hattori-Stallings rank was meant to avoid.

Thus, in the following, in the case of the height based in the Hattori-Stallings rank, we will simply restrict to the archimedean part. 


\section{More General AlgEBraS}

In the previous section we have taken inspiration from the setting of [6], designed to provide a noncommutative geometry setting for the Arakelov geometry of noncommutative arithmetic curves. When we try to extend these ideas beyond the case of zero-dimensional varieties over a number field $\mathbb{K}$ (hence one-dimensional varieties over $\left.\operatorname{Spec}\left(\mathcal{O}_{\mathbb{K}}\right)\right)$ and their noncommutative counterparts, we encounter a more serious problem in how to introduce the data at infinity, that is, the contribution of the archimedean places. In the case of arithmetic surfaces (curves over a number field $\mathbb{K}$, seen as surfaces over $\operatorname{Spec}\left(\mathcal{O}_{\mathbb{K}}\right)$ ), noncommutative geometry was used in different ways: in [12], [13], [42] noncommutative geometry was used to model the fiber at infinity of an arithmetic surface, following a model developed in [40] based on Schottky uniformization and geodesics in a hyperbolic handlebody (see also [41] for a physical interpretation in the setting of AdS/CFT holography). In [7] noncommutative geometry is used in a different way, by developing noncommutative versions of arithmetic surfaces, based on noncommutative projective schemes. In the context of Arakelov geometry it is natural to work with projective schemes, because one is interested primarily in developing a good intersection theory. However, from the point of view of noncommutative geometry this creates complications in relating the algebro-geometric noncommutative projective schemes to analytic noncommutative spaces that should provide the data at the archimedean places. While this problem is solved in the case of noncommutative arithmetic surfaces in [7] by a suitable definition of hermitian bundles, we take here a different viewpoint on noncommutative arithmetic spaces, which is based on affine geometry. This is sufficient for our purposes, and it will have the advantage that it makes more direct and transparent the reole of the archimedean places.

5.1. Arithmetic noncommutative spaces. For our purposes, we take the following definition of an arithmetic noncommutative space.

Definition 5.1. Let $\mathbb{K}$ be a number field and $\mathcal{O}_{\mathbb{K}}$ its ring of integers. An arithmetic noncommutative space in arbitrary dimension is given by the following data.

(1) $A$ is a finitely generated associative algebra over $\mathbb{K}$.

(2) $\mathcal{O}_{A}$ is a subring of $A$ with the structure of flat $\mathcal{O}_{\mathbb{K}}$-module.

(3) As $\mathbb{K}$-vector spaces $A=\mathcal{O}_{A} \otimes_{\mathcal{O}_{\mathbb{K}}} \mathbb{K}$.

(4) For every archimedean place $\sigma: \mathbb{K} \hookrightarrow \mathbb{K}_{\sigma}$ with $\mathbb{K}_{\sigma}=\mathbb{R}$ or $\mathbb{C}$, the real/complex algebra $A_{\sigma}=A \otimes_{\mathbb{K}, \sigma} \mathbb{K}_{\sigma}$ has an involution $\star$.

(5) $A_{\sigma}$, for $\sigma$ complex, and $A_{\sigma} \otimes_{\mathbb{R}} \mathbb{C}$ for $\sigma$ real, are dense involutive subalgebras of a complex $C^{*}$-algebra $\bar{A}_{\sigma}$.

(6) There is a normalized trace $\tau_{\sigma}: \bar{A}_{\sigma} \rightarrow \mathbb{K}_{\sigma}$ such that $\left.\tau_{\sigma}\right|_{\mathcal{O}_{A}}$ takes values in $\mathcal{O}_{\mathbb{K}}$.

This notion of arithmetic noncommutative spaces only involves Type II algebras. Thus, for example, certain noncommutative spaces of arithmetic relevance such as the endomotives of [9], including the Bost-Connes type algebras over number fields of [58], are not included in this definition. While such algebras certainly should be 
regarded as arithmetic spaces, they would not be of finite type over $\operatorname{Spec}\left(\mathcal{O}_{\mathbb{K}}\right)$, while our notion is meant to capture this finite type condition.

Example 5.2. The arithmetic noncommutative torus is the finitely generated algebra $A\left(\mathbb{T}_{\theta}\right)$ over $\mathbb{Q}$ with generators $U, V, U^{*}, V^{*}$ and relations $U^{*} U=U U^{*}=1$, $V^{*} V=V V^{*}=1$ and $V U=e^{2 \pi i \theta} U V$ for some fixed $\theta \in \mathbb{R}$. The subring $\mathcal{O}_{A\left(\mathbb{T}_{\theta}\right)}$ is generated over $\mathbb{Z}$ by the same generators and relations. As a $\mathbb{Z}$-module it is the span $\mathbb{Z}\left\langle U^{n} V^{m} \mid n, m \in \mathbb{Z}\right\rangle$, which is an abelian group with no torsion, hence a flat $\mathbb{Z}$-module. For the unique real embedding of $\mathbb{Q}$ the algebra $A\left(\mathbb{T}_{\theta}\right)_{\sigma} \otimes_{\mathbb{R}} \mathbb{C}$ is a dense subalgebra of the usual $C^{*}$-algebra of the noncommutative torus. The trace $\tau\left(U^{n} V^{m}\right)=\delta_{n, 0} \delta_{m, 0}$ $\operatorname{maps} \mathcal{O}_{A\left(\mathbb{T}_{\theta}\right)}$ to $\mathbb{Z}$.

For two $C^{*}$-algebras $\bar{A}$ and $\bar{B}$, we recall the notion of Hilbert $\bar{A}-\bar{B}$ bimodule, [25].

Definition 5.3. A Hilbert $\bar{A}-\bar{B}$ bimodule $H$ of finite type is defined by the following properties:

- $H$ is an $\bar{A}-\bar{B}$-bimodule, which is finitely generated projective as a right $\bar{B}$-module and as a left $\bar{A}$-module

- $H$ is self-dual, with respect to the duality given by the conjugate bimodule.

- There are an $\bar{A}$-valued inner product $\bar{A}\langle\cdot, \cdot\rangle$ and a $\bar{B}$-valued inner product $\langle\cdot, \cdot\rangle_{\bar{B}}$, with the first left-linear and right-conjugate-linear and the second leftconjugate-linear and right-linear.

- For all $a \in \bar{A}, b \in \bar{B}$, and $x, y \in H$

$$
\begin{aligned}
& { }_{\bar{A}}\langle a x, y\rangle=a_{\bar{A}}\langle x, y\rangle, \quad \text { and } \quad{ }_{\bar{A}}\langle x, a y\rangle={ }_{\bar{A}}\langle x, y\rangle a^{*} \\
& \langle x, y b\rangle_{\bar{B}}=\langle x, y\rangle_{\bar{B}} b \quad \text { and } \quad\langle x b, y\rangle_{\bar{B}}=b^{*}\langle x, y\rangle_{\bar{B}}
\end{aligned}
$$

- For all $x \in H, \bar{A}\langle x, x\rangle \geq 0$ with $\bar{A}\langle x, x\rangle=0$ iff $x=0$ and $\langle x, x\rangle_{\bar{B}} \geq 0$ with $\langle x, x\rangle_{\bar{B}}=0$ iff $x=0$.

- For all $x, y \in H$,

$$
\bar{A}\langle x, y\rangle={ }_{\bar{A}}\langle y, x\rangle^{*} \quad \text { and } \quad\langle x, y\rangle_{\bar{B}}=\langle y, x\rangle_{\bar{B}}^{*}
$$

- The norms $\|x\|_{A}:=\|\|_{\bar{A}}\langle x, x\rangle \|_{\bar{A}}^{1 / 2}$ and $\|x\|_{B}:=\left\|\langle x, x\rangle_{\bar{B}}\right\|_{\bar{B}}^{1 / 2}$ satisfy an estimate

$$
C_{1}\|x\|_{A} \leq\|x\|_{B} \leq C_{2}\|x\|_{A}
$$

for some constants $C_{1}, C_{2}>0$ and for all $x \in H$.

- $H$ is complete with respect to the $\|\cdot\|_{A}$ norm (equivalently, the $\|\cdot\|_{B}$ norm).

Remark 5.4. The finite type condition, that $H$ is finitely generated projective both as a right $\bar{B}$-module and as a left $\bar{A}$-module, and that it is self-dual with respect to conjugation is equivalent to the condition that there are a left $\bar{A}$-basis $\left\{v_{1}, \ldots, v_{m}\right\}$ and a right $\bar{B}$-basis $\left\{u_{1}, \ldots, u_{n}\right\}$ such that, for all $x \in H$,

$$
x=\sum_{i=1}^{m} \bar{A}\left\langle x, v_{i}\right\rangle v_{i} \quad \text { and } \quad x=\sum_{j=1}^{n} u_{j}\left\langle u_{j}, x\right\rangle_{\bar{B}} .
$$

See Lemma 1.7 of [25], and see also the comment on duality on p. 3443 of [25]. 
In terms of a right $\bar{B}$-basis $\left\{u_{1}, \ldots, u_{n}\right\}$, the structure of $H$ as a finitely generated projective right $\bar{B}$-module can be seen explicitly by identifying $H \simeq p \bar{B}^{n}$, where $p \in M_{n}(\bar{B})$ is a projection given by $p_{i j}=\left\langle u_{i}, u_{j}\right\rangle_{\bar{B}}$. The maps from $H$ to $p \bar{B}^{n}$ and viceversa are given by $x \mapsto\left(\left\langle u_{i}, x\right\rangle_{\bar{B}}\right)_{i}$, which maps $H$ to the range of $p$ in $\bar{B}^{n}$, and its inverse map $\left(y_{i}\right) \mapsto \sum_{i} u_{i} y_{i}$ from $p \bar{B}^{n}$ to $H$. (See Lemma 1.11 of [25].) Also note that the condition on the equivalence of the norms $\|x\|_{A}$ and $\|x\|_{B}$ in Definition 5.3 and of completeness in these norms follow automatically from the finite type condition, as in Remark 5.4 (see Lemma 1.11 and Proposition 1.18 of [25].)

We can then generalize the definition of hermitian bimodules given in Definition 4.6 in the following way.

Definition 5.5. Let $\left(A, \mathcal{O}_{A}\right)$ and $\left(B, \mathcal{O}_{B}\right)$ be arithmetic noncommutative spaces as in Definition 5.1. The category ${ }_{\left(A, \mathcal{O}_{A}\right)} \mathcal{H}_{\left(B, \mathcal{O}_{B}\right)}$ of arithmetic hermitian bimodules of finite type has objects $\left(\mathcal{E}, h_{A}, h_{B}\right)$ satisfying all the properties of Definition 4.6 as well as the following:

- $\mathcal{E}$ is an $\mathcal{O}_{A}-\mathcal{O}_{B}$ bimodule, and a left-right $\mathcal{O}_{\mathbb{K}^{-}}$lattice, such that $A \otimes_{\mathcal{O}_{A}} \mathcal{E}$ is finitely generated projective as an $A$-module and $\mathcal{E} \otimes_{\mathcal{O}_{B}} B$ is finitely generated projective as $B$-module.

- for any archimedean place $\sigma$ of $\mathbb{K}$ the bimodule $\mathcal{E}_{\sigma}:=A_{\sigma} \otimes_{\mathcal{O}_{A}} \mathcal{E} \otimes_{\mathcal{O}_{B}} B_{\sigma}$ is endowed with a pair $h=\left(h_{A}, h_{B}\right)$ with $h_{A}: \mathcal{E}_{\sigma} \times \mathcal{E}_{\sigma} \rightarrow A_{\sigma}$ and $h_{B}: \mathcal{E}_{\sigma} \times \mathcal{E}_{\sigma} \rightarrow$ $B_{\sigma}$

- $h_{A}$ is left-linear and right-conjugate-linear and defines an $A_{\sigma}$-valued inner product on $\mathcal{E}_{\sigma}$.

- $h_{B}$ is left-conjugate-linear and right-linear and defines a $B_{\sigma}$-valued inner product on $\mathcal{E}_{\sigma}$.

- There is a Hilbert $\bar{A}_{\sigma}-\bar{B}_{\sigma}$ bimodule of finite type $\overline{\mathcal{E}}_{\sigma}$ with $\bar{A}_{\sigma}\langle x, y\rangle=h_{A}(x, y)$ and $\langle x, y\rangle_{\bar{B}_{\sigma}}=h_{B}(x, y)$

- $\overline{\mathcal{E}}_{\sigma}$ is the completion of $\mathcal{E}_{\sigma}$ in the norm $\|x\|_{A}=\left\|h_{A}(x, x)\right\|_{\bar{A}_{\sigma}}^{1 / 2}$ (equivalently, in the norm $\left.\|x\|_{B}=\left\|h_{B}(x, x)\right\|_{\bar{B}_{\sigma}}^{1 / 2}\right)$.

Morphisms $\phi:\left(\mathcal{E}, h_{A}, h_{B}\right) \rightarrow\left(\mathcal{E}^{\prime}, h_{A}^{\prime}, h_{B}^{\prime}\right)$ are morphisms $\phi: \mathcal{E} \rightarrow \mathcal{E}^{\prime}$ of $\mathcal{O}_{A}-\mathcal{O}_{B}$ bimodules such that the induced map $\phi_{\sigma}: \overline{\mathcal{E}}_{\sigma} \rightarrow \overline{\mathcal{E}}_{\sigma}^{\prime}$ is a finite rank operator that preserves the hermitian structures, $h_{A}^{\prime}\left(\phi_{\sigma}(x), \phi_{\sigma}(y)\right)=h_{A}(x, y)$ and $h_{B}^{\prime}\left(\phi_{\sigma}(x), \phi_{\sigma}(y)\right)=$ $h_{B}(x, y)$.

With respect to a right $\bar{B}$-basis $\left\{u_{1}, \ldots, u_{n}\right\}$ of $\overline{\mathcal{E}}_{\sigma}$ a bimodule homomorphism $\phi_{\sigma}: \overline{\mathcal{E}}_{\sigma} \rightarrow \overline{\mathcal{E}}_{\sigma}^{\prime}$ satisfies

$$
\phi_{\sigma}(x)=\sum_{i=1}^{n} T\left(u_{i}\right)\left\langle u_{i}, x\right\rangle_{\bar{B}_{\sigma}},
$$

and similarly for a left $\bar{A}$-basis $\left\{v_{1}, \ldots, v_{m}\right\}$ of $\overline{\mathcal{E}}_{\sigma}$. Thus, for the condition that morphisms preserve the hermitian structure it suffices that on the basis elements $h_{B}\left(\phi_{\sigma}\left(u_{i}\right), \phi_{\sigma}\left(u_{j}\right)\right)=h_{B}\left(u_{i}, u_{j}\right)$ and $h_{A}\left(\phi_{\sigma}\left(v_{i}\right), \phi_{\sigma}\left(v_{j}\right)\right)=h_{A}\left(v_{i}, v_{j}\right)$. 
Remark 5.6. The functor of points of an arithmetic noncommutative space $\left(A, \mathcal{O}_{A}\right)$ assigns to $\left(B, \mathcal{O}_{B}\right)$ the category ${ }_{\left(A, \mathcal{O}_{A}\right)} \mathcal{H}_{\left(B, \mathcal{O}_{B}\right)}$ of arithmetic hermitian bimodules of finite type as the $\left(B, \mathcal{O}_{B}\right)$ points of $\left(A, \mathcal{O}_{A}\right)$, and to $\left(B, \mathcal{O}_{B}\right)-\left(B^{\prime}, \mathcal{O}_{B}^{\prime}\right)$ bimodules $\mathcal{F}$ the functor $\mathcal{F}:{ }_{\left(A, \mathcal{O}_{A}\right)} \mathcal{H}_{\left(B, \mathcal{O}_{B}\right)} \rightarrow{ }_{\left(A, \mathcal{O}_{A}\right)} \mathcal{H}_{\left(B^{\prime}, \mathcal{O}_{B}^{\prime}\right)}$ given by tensoring with $\mathcal{F}$.

5.2. The case of noncommutative tori. We consider again the example of arithmetic structures on noncommutative tori, mentioned in Example 5.2 above.

The Rieffel construction of finite projective modules on noncommutative tori [50], [51] is based on using imprimitivity bimodules. These are Hilbert bimodules ${ }_{A} \mathcal{F}_{B}$, for unital $C^{*}$-algebras $A, B$, with the property that the $C^{*}$-algebra valued inner products satisfy the relation

$$
\langle x, y\rangle_{A} z=x_{B}\langle y, z\rangle
$$

for all $x, y, z \in{ }_{A} \mathcal{F}_{B}$. An imprimitivity bimodule implements a strong Morita equivalence between the $C^{*}$-algebras $A$ and $B$, 552. Thus, these are regarded as a good notion of "isomorphism" of the corresponding non-commutative spaces, in a setting where morphisms are defined by (some class of) Hilbert bimodules. The construction of projective modules is based on the observation that, if ${ }_{A} \mathcal{F}_{B}$ is an imprimitivity bimodule and $p$ is a projection in $A$, then $p \mathcal{F}$ is a projective $B$-module, since there will be two sets $\left\{x_{i}\right\}_{i=1}^{n}$ and $\left\{y_{i}\right\}_{i=1}^{n}$ of elements of $\mathcal{F}$ such that

$$
\sum_{i}\left\langle x_{i}, y_{i}\right\rangle_{A}=p
$$

Then for each $z \in p \mathcal{F}$ one has $z=p z=\sum_{i}\left\langle x_{i}, y_{i}\right\rangle_{A} z=\sum_{i} x_{i B}\left\langle y_{i}, z\right\rangle$ so that the $x_{i}$ give a finite set of generators for $p \mathcal{F}$ and the maps

$$
\begin{aligned}
& \Psi: B^{n} \rightarrow p \mathcal{F} \quad \text { and } \quad \Phi: p \mathcal{F} \rightarrow B^{n} \\
& \Psi:\left(b_{i}\right) \mapsto \sum_{i} x_{i} b_{i} \text { and } \Phi: z \mapsto\left({ }_{B}\left\langle y_{i}, z\right\rangle\right)
\end{aligned}
$$

identify $p \mathcal{F}$ with a direct summand of the free module $B^{n}$, so that $p \mathcal{F}$ is indeed a projective $B$-module, see Proposition 1.2 of [51]. Thus, the construction of projective $B$-modules follows from the construction of projections $p$ in the Morita-equivalent algebra $A$.

In particular, an arithmetic structure on a projective module $X=p \mathcal{F}$ over a noncommutative torus $B=A\left(\mathbb{T}_{\theta}\right)$ is obtained by considering the image $\Psi\left(\mathcal{O}_{A\left(\mathbb{T}_{\theta}\right)}\right) \subset$ $p \mathcal{F}$,

$$
\begin{aligned}
\Psi\left(\mathcal{O}_{A\left(\mathbb{T}_{\theta}\right)}\right) & =\left\{\xi \in p \mathcal{F} \mid \xi=\sum_{i} x_{i} b_{i}, \text { with } b_{i} \in \mathcal{O}_{A\left(\mathbb{T}_{\theta}\right)}\right\} \\
& =\left\{\left.\xi \in p \mathcal{F}\right|_{A\left(\mathbb{T}_{\theta}\right)}\left\langle y_{i}, \xi\right\rangle \in \mathcal{O}_{A\left(\mathbb{T}_{\theta}\right)}\right\}
\end{aligned}
$$

The construction of projections in a noncommutative torus $A=A\left(\mathbb{T}_{\theta}\right)$ is obtained as in [50] by considering the $A$-valued inner product $\langle\xi, \xi\rangle_{A}$ of elements $\xi \in{ }_{A} \mathcal{F}_{B}$. The resulting $p_{\xi}=\langle\xi, \xi\rangle_{A}$ is a projection if and only if it satisfies the identity $\xi_{B}\langle\xi, \xi\rangle=\xi$. Indeed, one can see that

$$
p_{\xi}^{2}=\langle\xi, \xi\rangle_{A}\langle\xi, \xi\rangle_{A}=\left\langle\langle\xi, \xi\rangle_{A} \xi, \xi\right\rangle_{A}=\left\langle\xi{ }_{B}\langle\xi, \xi\rangle, \xi\right\rangle_{A}=\langle\xi, \xi\rangle_{A}=p_{\xi}
$$


and $p_{\xi}^{*}=p_{\xi}$. The converse follows similarly (see Lemma 3.2 of [35]). There are several explicit constructions of projections in noncommutative tori. In particular, Boca [4] presented a construction based on theta functions, and Luef and Manin [36] and Luef [35] gave a general construction based on Gabor frames.

One can then obtain hermitian bimodules of finite type in the following way. Here we assume we have fixed a specific construction that associates to a pair of Morita equivalent $C^{*}$-algebras of irrational noncommutative tori and an imprimitivity bimodule implementing the Morita equivalence a projection in one of the two $C^{*}$-algebras obtained as described above.

Proposition 5.7. Let $A_{\theta}=A\left(\mathbb{T}_{\theta}\right)$ and $A_{\theta^{\prime}}=A\left(\mathbb{T}_{\theta^{\prime}}\right)$ be the $C^{*}$-algebras of two irrational noncommutative tori. Let $\tilde{\theta}$ and $\tilde{\theta}^{\prime}$ be a choice of points in the $\mathrm{GL}_{2}(\mathbb{Z})$ orbits of $\theta$ and $\theta^{\prime}$ respectively, under the action on $\mathbb{P}^{1}(\mathbb{R})$ by fractional linear transformations. Such a choice determines an $\left(A_{\theta}, \mathcal{O}_{A_{\theta}}\right)-\left(A_{\theta^{\prime}}, \mathcal{O}_{A_{\theta^{\prime}}}\right)$ hermitian bimodule as in Definition 5.5.

Proof. Since $\theta$ and $\tilde{\theta}$ (respectively, $\theta^{\prime}$ and $\tilde{\theta}^{\prime}$ ) are in the same $\mathrm{GL}_{2}(\mathbb{Z}$ ) orbit by fractional linear transformations, the $C^{*}$-algebras $A_{\theta}$ and $A_{\tilde{\theta}}$ (respectively, $A_{\theta^{\prime}}$ and $A_{\tilde{\theta}^{\prime}}$ ) are Morita equivalent, with the equivalence implemented by imprimitivity bimodules $A_{\theta} \mathcal{F}_{A_{\tilde{\theta}}}$ and ${ }_{A_{\tilde{\theta}}} \mathcal{F}_{A_{\theta^{\prime}}}^{\prime}$. Let $p_{\tilde{\theta}}$ and $p_{\tilde{\theta}^{\prime}}$ be projections in $A_{\tilde{\theta}}$ and $A_{\tilde{\theta}^{\prime}}$, respectively, and let $X=\mathcal{F} p_{\tilde{\theta}}$ and $X^{\prime}=p_{\tilde{\theta}^{\prime}} \mathcal{F}^{\prime}$ be the corresponding projective modules, constructed as recalled above. Consider then the $A_{\theta}-A_{\theta^{\prime}}$ Hilbert bimodule $\mathcal{E}=X \otimes_{\mathbb{C}} X^{\prime}$. As above, let $\left\{x_{i}\right\}$ and $\left\{y_{i}\right\}$ be elements of $\mathcal{F}$ and $\left\{x_{j}^{\prime}\right\}$ and $\left\{y_{j}^{\prime}\right\}$ be elements of $\mathcal{F}^{\prime}$ such that

$$
\sum_{i} A_{\tilde{\theta}}\left\langle y_{i}, x_{i}\right\rangle=p_{\tilde{\theta}} \quad \text { and } \quad \sum_{j}\left\langle x_{j}^{\prime}, y_{j}^{\prime}\right\rangle_{A_{\tilde{\theta}^{\prime}}}=p_{\tilde{\theta}^{\prime}}
$$

Let $\Phi_{\tilde{\theta}}, \Psi_{\tilde{\theta}}$ and $\Phi_{\tilde{\theta}^{\prime}}, \Psi_{\tilde{\theta}^{\prime}}$ be the maps defined as in (5.3) with $\Psi_{\tilde{\theta}}: A_{\theta}^{n} \rightarrow X, \Psi_{\tilde{\theta}}\left(a_{i}\right)=$ $\sum_{i} a_{i} x_{i}$ and $\Psi_{\tilde{\theta}^{\prime}}: A_{\theta^{\prime}}^{n} \rightarrow X^{\prime}, \Psi_{\tilde{\theta}^{\prime}}\left(a_{j}^{\prime}\right)=\sum_{j} x_{j}^{\prime} a_{j}^{\prime}$. Elements $\xi \otimes \xi^{\prime} \in \mathcal{E}$ can be written as $\xi \otimes \xi^{\prime}=\sum_{i, j}\left\langle\xi, y_{i}\right\rangle_{A_{\theta}} x_{i} \otimes x_{j}^{\prime} A_{\theta^{\prime}}\left\langle y_{j}^{\prime}, \xi\right\rangle=\sum_{i, j} a_{i} x_{i} \otimes x_{j}^{\prime} a_{j}^{\prime}$. We define the arithmetic submodule $\mathcal{E}_{\mathbb{Z}}$ as in (5.4) by setting

$$
\mathcal{E}_{\mathbb{Z}}:=\left\{\xi \otimes \xi^{\prime}=\sum_{i, j} a_{i} x_{i} \otimes x_{j}^{\prime} a_{j}^{\prime} \mid a_{i} \in \mathcal{O}_{A_{\theta}}, a_{j}^{\prime} \in \mathcal{O}_{A_{\theta^{\prime}}}, \forall i, j\right\}
$$

We define hermitian structures $h_{\theta}$ and $h_{\theta^{\prime}}$ on $\mathcal{E}_{\mathbb{Z}}$ as $h_{\theta}(\xi, \eta)={ }_{A_{\theta}}\langle\xi, \eta\rangle$ and $h_{\theta^{\prime}}(\xi, \eta)=$ $\langle\xi, \eta\rangle_{A_{\theta^{\prime}}}$.

5.3. Height function as volume. We would like then to assign a height to the bimodules $\left(\mathcal{E}, h_{A}, h_{B}\right)$ constructed as above. We first discuss how to obtain a version of the height $H\left(\mathcal{E}, h_{A}, h_{B}\right)$ that corresponds to the expressions (4.2) and (4.9). We then discuss how to obtain a notion of height $\mathfrak{h}\left(\mathcal{E}, h_{A}, h_{B}\right)$ that generalized the one based on the Hattori-Stallings rank that we discussed in the previous section. 
We want to assign to an arithmetic hermitian bimodule of finite type $\left(\mathcal{E}, h_{A}, h_{B}\right)$ a height function of the form

$$
H\left(\mathcal{E}, h_{A}, h_{B}\right)=\frac{\operatorname{vol}_{B, h_{B}}(\mathcal{E})}{\operatorname{vol}_{A, h_{A}}(\mathcal{E})},
$$

as a direct analog of (4.9).

In order to obtain the volumes $\operatorname{vol}_{A, h_{A}}(\mathcal{E})$ and $\operatorname{vol}_{B, h_{B}}(\mathcal{E})$ we proceed as in the previous section. Let $\left(\mathcal{E}, h_{A}, h_{B}\right)$ be an arithmetic hermitian bimodule of finite type as in Definition 5.5. Let $\mathcal{B}_{A_{\sigma}}(\mathcal{E})$ and $\mathcal{B}_{B_{\sigma}}(\mathcal{E})$ be, respectively, the sets of left $\bar{A}_{\sigma}$-bases of $\mathcal{E}_{\sigma}\left\{v_{1}, \ldots, v_{m}\right\}$, with that $v_{j} \in \mathcal{E}$, and the set of right $\bar{B}_{\sigma}$-bases $\left\{u_{1}, \ldots, u_{n}\right\}$ of $\mathcal{E}_{\sigma}$ with $u_{i} \in \mathcal{E}$.

We define the volume via an optimization over the choice of basis. Namely, we set

$$
\begin{aligned}
\operatorname{vol}_{A, h_{A}}(\mathcal{E}) & :=\prod_{\sigma} \inf _{\mathcal{B}_{A_{\sigma}}(\mathcal{E})} \prod_{j} \tau_{A_{\sigma}}\left(h_{A}\left(v_{j}, v_{j}\right)\right), \\
\operatorname{vol}_{B, h_{B}}(\mathcal{E}) & :=\prod_{\sigma} \inf _{\mathcal{B}_{B_{\sigma}}(\mathcal{E})} \prod_{i} \tau_{B_{\sigma}}\left(h_{B}\left(u_{i}, u_{i}\right)\right),
\end{aligned}
$$

where $\tau_{A_{\sigma}}, \tau_{B_{\sigma}}$ denote the unique trace on the algebras $A_{\sigma}, B_{\sigma}$.

This provides an analog of (4.9) extending the notion of height derived from (4.2). However, as in the case of (4.9), the height function obtained in this way is not well behaved with respect to taking tensor products of bimodules, hence it is not directly compatible with the categorical composition operation on our noncommutative notion of "points". To avoid this problem, we consider, as in the previous section, a different notion of height based on the appropriate notion of "dimension", instead of a height function based on volumes.

5.4. Jones index as a height function. We return here to the general setting of Definition 5.3. The case of noncommutative tori discussed above is included as a special case.

Let $\left(\mathcal{E}, h_{A}, h_{B}\right)$ be a arithmetic hermitian bimodule of finite type as in Definition 5.5 and let $\left\{u_{1}, \ldots, u_{n}\right\}$ and $\left\{v_{1}, \ldots, v_{m}\right\}$ be, respectively, a right $\bar{B}_{\sigma^{-}}$basis and a left $\bar{A}_{\sigma^{-}}$ basis. The right and left index are defined as the elements

$$
\operatorname{Ind}_{A, \sigma}(\mathcal{E}):=\sum_{i} h_{A}\left(u_{i}, u_{i}\right) \in Z\left(\bar{A}_{\sigma}\right) \quad \text { and } \quad \operatorname{Ind}_{B, \sigma}(\mathcal{E}):=\sum_{j} h_{B}\left(v_{j}, v_{j}\right) \in Z\left(\bar{B}_{\sigma}\right)
$$

with $Z\left(\bar{A}_{\sigma}\right)$ and $Z\left(\bar{B}_{\sigma}\right)$ the centers of the respective $C^{*}$-algebras. These elements are independent of the choice of basis (Proposition 1.13 of [25]). We use here the notation $\operatorname{Ind}_{A, \sigma}(\mathcal{E})$ and $\operatorname{Ind}_{B, \sigma}(\mathcal{E})$ for what would be, respectively, $\operatorname{Ind}^{R}\left(\mathcal{E}_{\sigma}\right)$ and $\operatorname{Ind}^{L}\left(\mathcal{E}_{\sigma}\right)$ in the notation of [25].

Consider algebras $A, B$ such that $Z\left(A_{\sigma}\right)=Z\left(B_{\sigma}\right)=\mathbb{K}_{\sigma}$ for all archimedean places $\sigma$. Then the Jones index is multiplicative over tensor product of bimodules $\operatorname{Ind}_{A, \text { sigma }}\left(\mathcal{E} \otimes_{B} \mathcal{F}\right)=\operatorname{Ind}_{A, \text { sigma }}(\mathcal{E}) \cdot \operatorname{Ind}_{B, \sigma}(\mathcal{F}), \quad \operatorname{Ind}_{C, \sigma}\left(\mathcal{E} \otimes_{B} \mathcal{F}\right)=\operatorname{Ind}_{B, \sigma}(\mathcal{E}) \cdot \operatorname{Ind}_{C, \sigma}(\mathcal{F})$, 
see Proposition 1.30 of [25].

For algebras with $Z\left(A_{\sigma}\right)=Z\left(B_{\sigma}\right)=\mathbb{K}_{\sigma}$ one can then define a height function as in (4.17)

$$
\mathfrak{h}\left(\mathcal{E}, h_{A}, h_{B}\right):=\prod_{\sigma} \frac{\operatorname{Ind}_{B, \sigma}(\mathcal{E})}{\operatorname{Ind}_{A, \sigma}(\mathcal{E})} .
$$

Imprimitivity bimodules $\left(\mathcal{E}, h_{A}, h_{B}\right)$ that implement a Morita equivalence between $A_{\sigma}$ and $B_{\sigma}$, have $\operatorname{Ind}_{B, \sigma}(\mathcal{E})=1=\operatorname{Ind}_{A, \sigma}(\mathcal{E})$, see Corollary 1.19 of [25]. Thus, imprimitivity bimodules are fixed points of the time evolution generated by the height function (5.8) as in Proposition 4.26,

The height zeta function associated to the height function (5.8) as discussed in $\$ 4.13$ involves bimodules with fixed values of the Jones index. This shows that the problem of studying "points of bounded height" in this setting is potentially an interesting question with several possible connections to other areas of mathematics. In the von Neumann algebra setting, the related question of classifying subfactors of fixed index is solved for index less than 4 (see [47]) where the values of the Jones index are quantized, and also for index between 4 and 5 (see [22]) where after excluding an infinite family, one can again reduce the question to discrete data, while the question becomes intractable for higher values of the index for the reasons explained in [22].

Acknowledgment. The second author is partially supported by NSF grant DMS1707882, and by NSERC Discovery Grant RGPIN-2018-04937 and Accelerator Supplement grant RGPAS-2018-522593.

\section{REFERENCES}

[1] J.C. Álvarez Paiva, A.C. Thompson, Volumes on normed and Finsler spaces, in "RiemannFinsler Geometry" MSRI Publications, Vol.50, 2004, 1-48.

[2] Y. André, Une introduction aux motifs (motifs purs, motifs mixtes, périodes), Panoramas et Synthèses, Vol. 17. Société Mathématique de France, 2004.

[3] M. Artin, J.J. Zhang, Noncommutative projective schemes, Adv. Math. 109 (1994), 228-287.

[4] F. Boca, Projections in rotation algebras and theta functions, Comm. Math. Phys., 202 (1999) N.2, 325-357.

[5] E. Bombieri, W. Gubler, Heights in Diophantine geometry, New Mathematical Monographs, 4. Cambridge University Press, 2006.

[6] T. Borek, Arakelov theory of noncommutative arithmetic curves, J. Number Theory 131 (2011), no. 2, 212-227.

[7] T. Borek, Arakelov theory of noncommutative arithmetic surfaces, J. Reine Angew. Math. 642 (2010), 37-55.

[8] A. Buss, R. Meyer, C. Zhu, A higher category approach to twisted actions on $C^{*}$-algebras, Proc. Edinb. Math. Soc. (2) 56 (2013) no. 2, 387-426.

[9] A. Connes, C. Consani, M. Marcolli, Noncommutative geometry and motives: the thermodynamics of endomotives, Adv. Math. 214 (2007), no. 2, 761-831.

[10] A. Connes, M. Marcolli, Noncommutative Geometry, Quantum Fields and Motives, Colloquium Publications, Vol. 55, American Mathematical Society, 2008

[11] A. Connes, M. Marcolli, Quantum Statistical Mechanics of $\mathbb{Q}$-lattices, in "Frontiers in number theory, physics, and geometry. I", pp. 269-347, Springer, 2006. 
[12] C. Consani, M. Marcolli, Noncommutative geometry, dynamics, and $\infty$-adic Arakelov geometry, Selecta Math. (N.S.) 10 (2004), no. 2, 167-251.

[13] C. Consani, M. Marcolli, New perspectives in Arakelov geometry, in "Number Theory", 81-102, CRM Proc. Lecture Notes, 36, Amer. Math. Soc., 2004.

[14] L. Dabrowski, T. Krajewski, G. Landi, Non-linear $\sigma$-models in noncommutative geometry: fields with values in finite spaces, in "Spacetime and fundamental interactions (Vietri sul Mare, 2003)". Modern Phys. Lett. A 18 (2003), no. 33-35, 2371-2379.

[15] L. Dabrowski, T. Krajewski, G. Landi, Some properties of nonlinear $\sigma$-models in noncommutative geometry, in "Proceedings of the 1999 Euroconference: On Non-commutative Geometry and Hopf Algebras in Field Theory and Particle Physics (Torino)", Internat. J. Modern Phys. B 14 (2000), no. 22-23, 2367-2382.

[16] L. Dabrowski, G. Landi, F. Luef, Sigma-model solitons on noncommutative spaces, Lett. Math. Phys. 105 (2015), no. 12, 1663-1688.

[17] Y. A. Drozd, V.V. Kirichenko, Finite-dimensional algebras, Springer, 1994.

[18] E. Ghate, E. Hironaka, The arithmetic and geometry of Salem numbers, Bull. Amer. Math. Soc. (N.S.) 38 (2001) no. 3, 293-314.

[19] A. Hattori, Rank element of a projective module, Nagoya Math. J. 25 (1965), 113-120.

[20] E. Hironaka, What is ... Lehmer's number? Notices Amer. Math. Soc. 56 (2009) no. 3, 374-375.

[21] N. Johnson, D. Johnson Yau, 2-Dimensional Categories. arXiv:2002.06055 (2020).

[22] V.F.R. Jones, S. Morrison, N. Snyder, The classification of subfactors of index at most 5, Bull. Amer. Math. Soc. (N.S.) 51 (2014), no. 2, 277-327.

[23] L. Kadison, The algebra of type $I I_{1}$ subfactors of finite index and the Jones polynomial, Talk at Lousiana State University, Baton Rouge, 1994 https://www . math.upenn.edu/ lkadison/SEMINAR.pdf

[24] L. Kadison, D. Kastler, Cohomological aspects and relative separability of finite Jones index subfactors, Nachr. Akad. Wiss. Göttingen Math. Phys. Kl. II (1992), no. 4, 11 pp.

[25] T. Kajiwara, Y. Watatani, Jones index theory by Hilbert $C^{*}$-bimodules and $K$-theory, Trans. Amer. Math. Soc. Vol. 352 (2000) N.8, 3429-3472.

[26] A. Kapustin, Topological strings on noncommutative manifolds, Int. J. Geom. Methods Mod. Phys. 1 (2004), no. 1-2, 49-81.

[27] K. Kato, Height functions for motives, Selecta Math. (N.S.) 24 (2018) no. 1, 403-472.

[28] R. Kenyon, A. Okounkov, S. Sheffield, Dimers and amoebae, Ann. of Math. (2) 163 (2006) no. $3,1019-1056$.

[29] T.Y. Lam, A first course in noncommutative rings, Graduate Texts in Mathematics, Vol. 131, Springer Verlag, 1991.

[30] A.K. Lenstra, H.W. Lenstra, L.Lovász, Factoring polynomials with rational coefficients, Mathematische Annalen, Vol. 261 (1982) N.4, 515-534.

[31] C. Liebendörfer, Linear equations and heights over division algebras, J. Number Theory 105 (2004), no. 1, 101-133.

[32] C. Liebendörfer, G. Rémond, Hauteurs de sous-espaces sur les corps non commutatifs, Math. Z. 255 (2007) 549-577.

[33] C. Liebendörfer, G. Rémond, Le théorème de Schanuel pour un corps non commutatif, Rend. Semin. Mat. Univ. Padova 130 (2013), 221-282.

[34] J. López Peña, O. Lorscheid, Mapping $\mathbb{F}_{1}$-land: an overview of geometries over the field with one element, in "Noncommutative geometry, arithmetic, and related topics", pp. 241-265, Johns Hopkins Univ. Press, 2011.

[35] F. Luef, Projections in noncommutative tori and Gabor frames, Proc. Amer. Math. Soc., Vol.139 (2011) N.2, 571-582.

[36] F. Luef, Yu.I. Manin, Quantum theta functions and Gabor frames for modulation spaces, Lett. Math. Phys., 88 (2009) N.1-3, 131-161. 
[37] Yu.I. Manin, Introduction to the theory of schemes, Translated from the Russian, edited and with a preface by Dimitry Leites. Moscow Lectures, 1. Springer, 2018.

[38] Yu.I. Manin, Problems on rational points and rational curves on algebraic varieties. Surveys in differential geometry, Vol. II (Cambridge, MA, 1993), 214-245, Int. Press, 1995.

[39] Yu.I. Manin, Lectures on zeta functions and motives (according to Deninger and Kurokawa), Astérisque 228 (1995) N.4, 121-163.

[40] Yu.I. Manin, Three-dimensional hyperbolic geometry as $\infty$-adic Arakelov geometry, Invent. Math. 104 (1991), 223-244.

[41] Yu.I. Manin, M. Marcolli, Holography principle and arithmetic of algebraic curves, Adv. Theor. Math. Phys. 5 (2001), no. 3, 617-650.

[42] M. Marcolli, Arithmetic Noncommutative Geometry, University Lecture Series, Vol. 36, American Mathematical Society, 2005.

[43] M. Marcolli, A. Zainy al-Yasry, Coverings, correspondences, and noncommutative geometry, J. Geom. Phys. 58 (2008), no. 12, 1639-1661.

[44] V. Mathai, J. Rosenberg, A noncommutative sigma-model, J. Noncommut. Geom. 5 (2011), no. $2,265-294$.

[45] J.C. McConnell, Division algebras - Beyond the quaternions, Amer. Math. Monthly 105 (1998), no. $2,154-162$.

[46] M.J. Mossinghoff, Polynomials with small Mahler measure, Math. of Computation, Vol.67 (1998) N.224, 1697-1705.

[47] A. Ocneanu, Quantized groups, string algebras and Galois theory for algebras, in "Operator algebras and applications", Vol. 2, volume 136 of London Math. Soc. Lecture Note Ser., 119172. Cambridge Univ. Press, 1988.

[48] E. Peyre, Beyond heights: slopes and distribution of rational points, arXiv:1806.11437

[49] P. Ribenboim, Equivalent forms of Hensel's Lemma, Expo. Math. 3 (1985), 3-24.

[50] M.A. Rieffel, $C^{*}$-algebras associated with irrational rotations, Pacific J. Math. Vol.93 (1981) N.2, 415-429.

[51] M.A. Rieffel, Projective modules over higher-dimensional non-commutative tori, Can. J. Math. Vol. XL (1988) N.2, 257-338.

[52] M.A. Rieffel, Induced representations of $C^{*}$-algebras, Adv. Math. 13 (1974) 176-257.

[53] W.M. Schmidt, On heights of algebraic subspaces and diophantine approximations, Ann. of Math. (2) 85 (1967), 430-472.

[54] J. Stienstra, Mahler measure, Eisenstein series and dimers, in "Mirror symmetry. V", pp.151158, AMS/IP Stud. Adv. Math., 38, Amer. Math. Soc., 2006.

[55] J. Stienstra, Mahler measure variations, Eisenstein series and instanton expansions, in "Mirror symmetry. V", pp. 139-150, AMS/IP Stud. Adv. Math., 38, Amer. Math. Soc., 2006.

[56] A.R. Wadsworth, Valuation theory on finite dimensional division algebras, in "Valuation theory and its applications", Vol. I (Saskatoon, SK, 1999), 385-449, Fields Inst. Commun., 32, Amer. Math. Soc., 2002.

[57] Y. Watatani, Index for $C^{*}$-subalgebras, Mem. Amer. Math. Soc. 83 (1990), no. 424.

[58] B. Yalkinoglu, On arithmetic models and functoriality of Bost-Connes systems, with an appendix by Sergey Neshveyev, Invent. Math. 191 (2013), no. 2, 383-425.

[59] A. Zahabi, Toric quiver asymptotics and Mahler measure: $\mathcal{N}=2$ BPS states, Journal of High Energy Physics (2019), Article number 121.

Perimeter Institute for Theoretical Physics, Waterloo, Canada

Department of Mathematics, The University of Chicago, Chicago, IL, USA

Email address: alima@uchicago.edu

Mathematics Department, California Institute of Technology, Pasadena, USA

Email address: matilde@caltech.edu 